\title{
First Asymmetric Synthesis of Orthoquinone Monoketal Enantiomers via Anodic Oxidation
}

\author{
Stéphane Quideau, ${ }^{*}$ Denis Deffieux and Isabelle Fabre
}

Institut Européen de Chimie et Biologie, 2 rue Robert Escarpit, 33607 Pessac cedex, France, and Centre de Recherche en Chimie Moléculaire, Laboratoire de Chimie des

Substances Végétales, Université Bordeaux 1, 351 cours de la Libération, 33405

Talence cedex, France

\section{Supporting Information}

\section{Experimental Section}

General Procedure. Acetonitrile $(\mathrm{MeCN})$, dichloromethane $\left(\mathrm{CH}_{2} \mathrm{Cl}_{2}\right)$, diethyl ether $\left(\mathrm{Et}_{2} \mathrm{O}\right)$, and methanol $(\mathrm{MeOH})$ were distilled under $\mathrm{N}_{2}$ immediately before use from $\mathrm{P}_{2} \mathrm{O}_{5}, \mathrm{CaH}_{2}$, and $\mathrm{CaCl}_{2}$, respectively. Tetrahydrofuran (THF) was purified by distillation from sodium/benzophenone under $\mathrm{N}_{2}$ immediately before use. Synthesis grade ethyl acetate was used as received. Light petroleum refers to the fraction boiling in the $40-60{ }^{\circ} \mathrm{C}$ boiling range. Moisture and oxygen sensitive reactions were carried out in flame-dried glassware under $\mathrm{N}_{2}$. Evaporations were conducted under reduced pressure at temperatures less than $45^{\circ} \mathrm{C}$ unless otherwise noted. Column chromatography was carried out under positive pressure using 40-60 $\mu \mathrm{m}$ silica gel (Merck) and the indicated solvents. Further drying of the residues was accomplished under high vacuum. Melting points are uncorrected. NMR spectra of samples in the indicated solvent were run at either 200, 250 or 300 MHz. Carbon multiplicities and stereochemical assignments were determined by DEPT135 and NOESY experiments, respectively. Electron impact (50-70 eV) and liquid secondary ion mass 
spectrometry low- and high-resolution data (EIMS, and LSIMS, HRMS) were obtained from the mass spectrometry laboratory of the CESAMO at the University of Bordeaux, Talence, France. Atmospheric pressure chemical ionization (APCI) mass spectrometry fragmentation data and MALDI-ToF high-resolution data (APCIMS and MALDI, HRMS) were obtained from the mass spectrometry laboratory at the European Institute of Chemistry and Biology, Pessac, France.

Preparation of the chiral alcohols 3 and 4. The Williamson-type etherification reaction between 5-bromoguaiacol (1) and (S)-2-chloro-1-phenylethanol (2) or its enantiomer $R$-(2) furnished mixtures of primary and secondary alcohol enantiomers in good yields (see Scheme below), probably via the styrene oxide intermediates derived from $S$-(2) or $R$-(2) - Both styrene oxides are commercially available under enantiomerically pure forms at lower costs than those of the chloroethanols $\mathbf{2}$, but lower yields and lower regioselectivity were observed in our first series of attempts to use them - Chromatographic separation of the mixtures afforded $R$-(3) and $S$-(4) in $28 \%$ and $48 \%$ yields from $S$-(2), and $S$-(3) and $R$-(4) in $18 \%$ and $42 \%$ yields from $R$-(2). The lack of better regioselectivity was somewhat disappointing, but the facility with which the primary and secondary alcohol products were separated offered us a convenient access to two pairs of enantiomers as starting materials for asymmetric anodic oxidation.

5-Bromoguaiacol (1). A 39\% solution of peracetic acid in acetic acid (30 mL, $446 \mathrm{mmol})$ was added dropwise to a stirring ice-cooled solution of 5-bromo-2-methoxybenzaldehyde (15.0 g, 70.0 mmol) in EtOAc $(160 \mathrm{~mL})$. The reaction mixture was allowed to warm up slowly to room temperature while stirred overnight, after which time it was diluted in $\mathrm{H}_{2} \mathrm{O}(80 \mathrm{~mL})$. After separation, the aqueous layer was extracted with EtOAc $(3 \times 20 \mathrm{~mL})$, and the combined organic layers were washed with saturated aqueous $\mathrm{NaHCO}_{3}(3 \times 10 \mathrm{~mL})$, dried over $\mathrm{Na}_{2} \mathrm{SO}_{4}$, filtered and evaporated. The yellow residue was dissolved in $\mathrm{MeOH}(160 \mathrm{~mL})$, then $\mathrm{KOH}$ pellets $(4.12 \mathrm{~g}, 73.6$ mmol) were added portionwise at $0^{\circ} \mathrm{C}$ under stirring. The reaction mixture was stirred at $0^{\circ} \mathrm{C}$ for an additional $30 \mathrm{~min}$, after which time it was diluted in $\mathrm{H}_{2} \mathrm{O}(80 \mathrm{~mL})$, slowly acidified with $10 \%$ aqueous $\mathrm{HCl}$, extracted with EtOAc $(3 \times 20 \mathrm{~mL})$, washed with brine $(2 \times 10 \mathrm{~mL})$, dried over $\mathrm{Na}_{2} \mathrm{SO}_{4}$, filtered and evaporated to give crude $\mathbf{1}$ as a yellow oil, which was purified by column 
chromatography, eluting with light petroleum/ $\mathrm{Et}_{2} \mathrm{O}(6: 1 \rightarrow 3: 1)$ to furnish $1(9.2 \mathrm{~g}, 65 \%)$ as an offwhite solid. All characterization data were identical to those previously reported. ${ }^{[1,2]}$

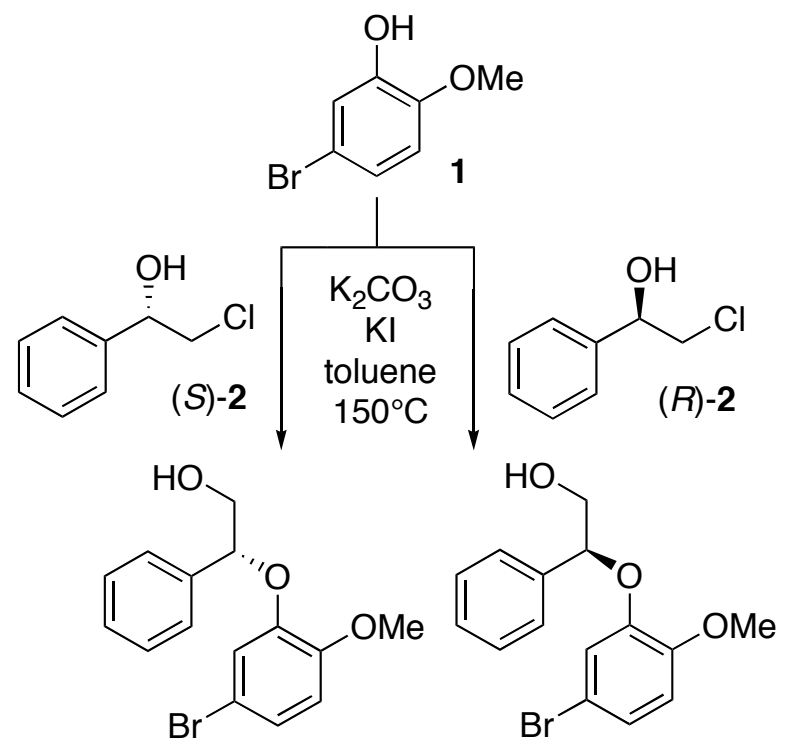

$(R)-3(28 \%)$

(S)-3 (18\%)<smiles>COc1ccc(Br)cc1OC[C@H](O)c1ccccc1</smiles>

\section{2-(5-Bromo-2-methoxyphenoxy)-(2R)-phenylethanol $[(R)-3]$ and 2-(5-Bromo-2-} methoxyphenoxy)-(1S)-phenylethanol $[(S)-4]$. To a stirred ice-cold solution of bromoguaiacol (1, $1.39 \mathrm{~g}, 6.9 \mathrm{mmol})$ in toluene $(30 \mathrm{~mL})$ were added powdered potassium carbonate $(4.60 \mathrm{~g}, 33.3$ mmol) and potassium iodine $(459 \mathrm{mg}, 2.7 \mathrm{mmol})$. After stirring for $15 \mathrm{~min},(S)-(+)$-2-chloro-1phenylethanol [S-(2), $1.42 \mathrm{~g}, 9.1 \mathrm{mmol}]$ was added. The reaction mixture was refluxed for 5 days, after which time toluene was evaporated. The residue was taken up with water $(20 \mathrm{~mL})$ and $\mathrm{CH}_{2} \mathrm{Cl}_{2}$ (30 mL). After separation, the aqueous phase was extracted with $\mathrm{CH}_{2} \mathrm{Cl}_{2}(3 \times 50 \mathrm{~mL})$. The combined organic layers were washed with brine, dried over $\mathrm{Na}_{2} \mathrm{SO}_{4}$, filtered, and evaporated to give a crude mixture of $(R)-3$ and $(S)-\mathbf{4}$, which was submitted to column chromatography, eluting with light petroleum/Et ${ }_{2} \mathrm{O}(3: 2)$, to furnish $(R)-3(622 \mathrm{mg}, 28 \%)$ and $(S)-4(1.07 \mathrm{~g}, 48 \%)$. $(R)-3$ : 
$[\alpha]^{21}{ }_{\mathrm{D}}=-44.68^{\circ}\left(\mathrm{c}=0.82, \mathrm{CH}_{2} \mathrm{Cl}_{2}\right) ; \mathrm{IR}(\mathrm{NaCl}) 3449,3053,2920,1588,1502 \mathrm{~cm}^{-1} ;{ }^{1} \mathrm{H} \mathrm{NMR}$ $\left(\mathrm{CDCl}_{3}, 250 \mathrm{MHz}\right) \delta 3.11(\mathrm{bs}, 1 \mathrm{H}), 3.75-3.99(\mathrm{~m}, 2 \mathrm{H}), 3.87(\mathrm{~s}, 3 \mathrm{H}), 5.10(\mathrm{dd}, J=8.5,3.4 \mathrm{~Hz}, 1 \mathrm{H})$, $6.75(\mathrm{~d}, J=8.5 \mathrm{~Hz}, 1 \mathrm{H}), 6.85(\mathrm{~d}, J=2.2 \mathrm{~Hz}, 1 \mathrm{H}), 7.04(\mathrm{dd}, J=8.7,2.3 \mathrm{~Hz}, 1 \mathrm{H}), 7.32-7.39(\mathrm{~m}, 5 \mathrm{H})$; ${ }^{13} \mathrm{C} \mathrm{NMR}\left(\mathrm{CDCl}_{3}, 62.9 \mathrm{MHz}\right) \delta 149.6,148.3,137.4,128.7,128.4,126.3,125.2,120.9,113.1$, 112.5, 85.0, 67.3, 56.0; EIMS m/z (rel intensity) $324\left(\mathrm{M}^{+}, 4\right), 322\left(\mathrm{M}^{+}, 4\right), 293$ (2), 291 (2), 277 (2), 275 (2), 204 (94), 202 (100), 189 (20), 187 (22); HRMS (EIMS) calcd for $\mathrm{C}_{15} \mathrm{H}_{15} \mathrm{O}_{3} \mathrm{Br} 322.0206$ found 322.0201. (S)-4: $[\alpha]^{21}{ }_{\mathrm{D}}=+14.02^{\circ}\left(\mathrm{c}=1.07, \mathrm{CH}_{2} \mathrm{Cl}_{2}\right)$; IR $(\mathrm{NaCl}) 3402,3054,2990,1508$, $1420 \mathrm{~cm}^{-1} ;{ }^{1} \mathrm{H}$ NMR $\left(\mathrm{CDCl}_{3}, 250 \mathrm{MHz}\right) \delta 3.78(\mathrm{~s}, 3 \mathrm{H}), 3.98(\mathrm{bt}, J=9.5 \mathrm{~Hz}, 1 \mathrm{H}), 4.11$ (dd, $J=9.8$, $2.8 \mathrm{~Hz}, 1 \mathrm{H}), 5.14(\mathrm{dd}, J=8.9,2.8 \mathrm{~Hz}, 1 \mathrm{H}), 6.73$ (d, $J=8.6 \mathrm{~Hz}, 1 \mathrm{H}), 7.00(\mathrm{dd}, J=1.8 \mathrm{~Hz}, 1 \mathrm{H}), 7.05$ $(\mathrm{dd}, J=8.5,2.1 \mathrm{~Hz}, 1 \mathrm{H}), 7.32-7.46(\mathrm{~m}, 5 \mathrm{H}) ;{ }^{13} \mathrm{C} \mathrm{NMR}\left(\mathrm{CDCl}_{3}, 50.3 \mathrm{MHz}\right) \delta 149.0,148.6,139.3$, 128.5, 128.1, 126.2, 124.6, 118.2, 113.0, 111.8, 75.7, 72.2, 56.0; EIMS $m / z$ (rel intensity) 324 (M+, 20), $322\left(\mathrm{M}^{+}, 20\right), 218$ (20), 216 (20), 204 (92), 202 (100), 189 (26), 187 (27); HRMS (EIMS) calcd for $\mathrm{C}_{15} \mathrm{H}_{15} \mathrm{O}_{3} \mathrm{Br} 322.0206$ found 322.0208 .

\section{2-(5-Bromo-2-methoxy-phenoxy)-(2S)-phenylethanol $[(S)-3]$ and 2-(5-Bromo-2-methoxy-}

phenoxy)-(1R)-phenylethanol $[(\boldsymbol{R})-4]$. To a stirred ice-cold solution of bromoguaiacol $(1,1.51 \mathrm{~g}$, $7.5 \mathrm{mmol})$ in toluene $(50 \mathrm{~mL})$ were added powdered potassium carbonate $(4.49 \mathrm{~g}, 32.5 \mathrm{mmol})$ and potassium iodine $(626 \mathrm{mg}, 3.8 \mathrm{mmol})$. After stirring for $15 \mathrm{~min},(R)-(-)$-2-chloro-1-phenylethanol $[R-(2), 1.19 \mathrm{~g}, 7.6 \mathrm{mmol}]$ was added. The reaction mixture was refluxed for 5 days, after which time toluene was evaporated. The residue was taken up with water $(20 \mathrm{~mL})$ and $\mathrm{CH}_{2} \mathrm{Cl}_{2}(30 \mathrm{~mL})$. After separation, the aqueous phase was extracted with $\mathrm{CH}_{2} \mathrm{Cl}_{2}(3 \times 50 \mathrm{~mL})$. The combined organic layers were washed with brine, dried over $\mathrm{Na}_{2} \mathrm{SO}_{4}$, filtered, and evaporated to give a crude mixture of $(S)$ 3 and $(R)-4$, which was submitted to column chromatography, eluting with light petroleum/Et $2 \mathrm{O}$ (3:2), to furnish $(S)-3(413 \mathrm{mg}, 18 \%)$ and $(R)-4(1.02 \mathrm{~g}, 42 \%) .(S)-3:[\alpha]^{21}{ }_{\mathrm{D}}=+43.02^{\circ}(\mathrm{c}=0.86$, $\left.\mathrm{CH}_{2} \mathrm{Cl}_{2}\right)$; IR ( $\left.\mathrm{NaCl}\right) 3373,2943,2830,1467,1420 \mathrm{~cm}^{-1} ;{ }^{1} \mathrm{H} \mathrm{NMR}\left(\mathrm{CDCl}_{3}, 250 \mathrm{MHz}\right) \delta 3.32$ (bs, 1H), $3.84(\mathrm{~s}, 3 \mathrm{H}), 3.74-4.00(\mathrm{~m}, 2 \mathrm{H}), 5.14(\mathrm{dd}, J=8.4,2.9 \mathrm{~Hz}, 1 \mathrm{H}), 6.73(\mathrm{~d}, J=8.5 \mathrm{~Hz}, 1 \mathrm{H}), 6.85$ $(\mathrm{s}, 1 \mathrm{H}), 7.02(\mathrm{~d}, J=8.9 \mathrm{~Hz}, 1 \mathrm{H}), 7.33-7.37(\mathrm{~m}, 5 \mathrm{H}) ;{ }^{13} \mathrm{C} \mathrm{NMR}\left(\mathrm{CDCl}_{3}, 62.9 \mathrm{MHz}\right) \delta 149.4,148.2$, 137.3, 128.6, 128.3, 126.2, 125.0, 120.4, 112.9, 112.4, 84.5, 67.2, 55.9; EIMS m/z (rel intensity) $324\left(\mathrm{M}^{+}, 17\right), 322\left(\mathrm{M}^{+}, 18\right), 218$ (26), 216 (27), 204 (90), 202 (100), 189 (26), 187 (27); HRMS 
(EIMS) calcd for $\mathrm{C}_{15} \mathrm{H}_{15} \mathrm{O}_{3} \mathrm{Br} 322.0206$ found 322.0195. $(R)-4:[\alpha]^{21}{ }_{\mathrm{D}}=-20.49^{\circ}\left(\mathrm{c}=1.22, \mathrm{CH}_{2} \mathrm{Cl}_{2}\right.$ ); IR (NaCl) 3304, 2946, 2827, $1450 \mathrm{~cm}^{-1} ;{ }^{1} \mathrm{H} \mathrm{NMR}\left(\mathrm{CDCl}_{3}, 250 \mathrm{MHz}\right) \delta 3.81$ (s, 3H), 3.98 (bt, $J=$ $9.5 \mathrm{~Hz}, 1 \mathrm{H}), 4.12(\mathrm{dd}, J=9.6,2.6 \mathrm{~Hz}, 1 \mathrm{H}), 5.14(\mathrm{dd}, J=9.0,2.6 \mathrm{~Hz}, 1 \mathrm{H}), 6.74(\mathrm{~d}, J=8.6 \mathrm{~Hz}, 1 \mathrm{H})$, $7.01(\mathrm{~s}, 1 \mathrm{H}), 7.07(\mathrm{~d}, J=8.6 \mathrm{~Hz}, 1 \mathrm{H}), 7.32-7.46(\mathrm{~m}, 5 \mathrm{H}) ;{ }^{13} \mathrm{C} \mathrm{NMR}\left(\mathrm{CDCl}_{3}, 62.9 \mathrm{MHz}\right) \delta 148.8$, $148.6,139.3,128.4,128.0,126.2,124.5,117.9,112.9,112.6,75.5,72.1,55.9$; EIMS m/z (rel intensity) $324\left(\mathrm{M}^{+}, 3\right), 322\left(\mathrm{M}^{+}, 3\right), 293$ (2), 291 (2), 204 (97), 202 (100), 189 (27), 187 (28); HRMS (EIMS) calcd for $\mathrm{C}_{15} \mathrm{H}_{15} \mathrm{O}_{3} \mathrm{Br} 322.0206$ found 322.0201 .

General Procedure for Anodic Oxidation. Electrolyses were carried out in a $100 \mathrm{~mL}$ divided cylindrical cell, equipped with a platinum grid as the anode and a platinum wire as the cathode. $\mathrm{KOH}$ pellets $(1.1 \mathrm{~g}, 19.6 \mathrm{mmol})$ was added as a supporting electrolyte into $100 \mathrm{~mL}$ of methanol. The starting alcohol was introduced into the anodic compartment, and the electrolysis was then performed on an Autolab PGSTAT 100 potentiostat using an $\mathrm{Ag} / \mathrm{AgCl}$ reference electrode. Electrolysis was carried out, under vigorous stirring, at a constant current of $c a$. $100 \mathrm{~mA}$, which was maintained at this value by increasing gradually the potential until TLC monitoring indicated the complete disappearance of the starting alcohol. The reaction mixture was then evaporated, and the residue was taken up in $\mathrm{CH}_{2} \mathrm{Cl}_{2}(50 \mathrm{~mL})$ and $\mathrm{H}_{2} \mathrm{O}(20 \mathrm{~mL})$. After separation, the aqueous layer was extracted with $\mathrm{CH}_{2} \mathrm{Cl}_{2}(3 \times 30 \mathrm{~mL})$ and the combined organic layers were washed with brine, dried over $\mathrm{Na}_{2} \mathrm{SO}_{4}$, filtered, and evaporated.

\section{9-Bromo-6,6-dimethoxy-(2R)-phenyl-1,4-dioxaspiro[4.(5S)]deca-7,9-diene [(RS)-5]. Anodic} oxidation of a solution of the primary alcohol $(R)-3(194 \mathrm{mg}, 0.6 \mathrm{mmol})$ in $\mathrm{MeOH}$ was performed according to the general procedure described above. Electrolysis was carried out at $100 \mathrm{~mA}$ by increasing potential from 2.80 to $3.90 \mathrm{~V} / \mathrm{Ag} / 0.1 \mathrm{M} \mathrm{AgCl}$. The reaction mixture was then processed as described above, and the residue was further dried overnight to give an oily crude product, which was purified by column chromatography, eluting with light petroleum/ $\mathrm{Et}_{2} \mathrm{O}$ (3:2), to furnish the pure spiro-bisketal $(R S)-5$ as a yellow oil $(71 \mathrm{mg}, 34 \%)$ : $[\alpha]^{21}{ }_{\mathrm{D}}=-6.67^{\circ}\left(\mathrm{c}=0.48, \mathrm{CH}_{2} \mathrm{Cl}_{2}\right)$; IR (NaCl) 2929, 2850, 1749, $1501 \mathrm{~cm}^{-1} ;{ }^{1} \mathrm{H}$ NMR $\left(\mathrm{CDCl}_{3}, 200 \mathrm{MHz}\right) \delta 3.45$ (s, 3H), 3.47 (s, 3H), 3.82-3.88 (m, 1H), 4.40 (bt, $J=7.2 \mathrm{~Hz}, 1 \mathrm{H}), 5.22(\mathrm{dd}, J=9.0,6.7 \mathrm{~Hz}, 1 \mathrm{H}), 5.89$ (d, $J=10.3 \mathrm{~Hz}$, 
$1 \mathrm{H}), 6.11(\mathrm{dd}, J=10.3,1.6 \mathrm{~Hz}, 1 \mathrm{H}), 6.35(\mathrm{~d}, J=1.4 \mathrm{~Hz}, 1 \mathrm{H}), 7.34-7.44(\mathrm{~m}, 5 \mathrm{H}) ;{ }^{13} \mathrm{C} \mathrm{NMR}\left(\mathrm{CDCl}_{3}\right.$, 75.5 MHz) $\delta 136.6,132.3,131.5,130.4,128.6,128.5,126.4,119.0,108.5,97.0,78.2,71.1,51.2$, 51.0; EIMS $m / z$ (rel intensity) $354\left(\mathrm{M}^{+}, 2\right), 352\left(\mathrm{M}^{+}, 2\right), 273$ (2), 250 (18), 248 (21); HRMS (EIMS) calcd for $\mathrm{C}_{16} \mathrm{H}_{17} \mathrm{O}_{4} \mathrm{Br} 352.0310$ found 352.0302 .

9-Bromo-6,6-dimethoxy-(2S)-phenyl-1,4-dioxaspiro[4.(5R)]deca-7,9-diene $[(S R)-5]$. Anodic oxidation of a solution of the primary alcohol $(S)-3(278 \mathrm{mg}, 0.9 \mathrm{mmol})$ in $\mathrm{MeOH}$ was performed according to the general procedure described above. Electrolysis was carried at $110 \mathrm{~mA}$ by increasing potential from 1.90 to $2.20 \mathrm{~V} / \mathrm{Ag} / 0.1 \mathrm{M} \mathrm{AgCl}$. The reaction mixture was then processed as described above, and the residue was further dried overnight to give an oily crude product, which was purified by column chromatography, eluting with light petroleum/ $\mathrm{Et}_{2} \mathrm{O}(3: 2 \rightarrow 1: 1)$, to furnish the pure spiro-bisketal $(S R)-5$ as a yellow oil $(83 \mathrm{mg}, 27 \%)$ : $[\alpha]^{21}{ }_{\mathrm{D}}=+0.88^{\circ}\left(\mathrm{c}=0.68, \mathrm{CH}_{2} \mathrm{Cl}_{2}\right)$; IR ( $\mathrm{NaCl}) 2938,2844,1694,1206 \mathrm{~cm}^{-1}$; ${ }^{1} \mathrm{H}$ NMR $\left(\mathrm{CDCl}_{3}, 250 \mathrm{MHz}\right) \delta 3.45(\mathrm{~s}, 3 \mathrm{H}), 3.47$ (s, 3H), 3.81 (bt, $J=8.2 \mathrm{~Hz}, 1 \mathrm{H}), 4.40$ (bt, $J=7.0 \mathrm{~Hz}, 1 \mathrm{H}), 5.22$ (dd, $J=9.2,6.7 \mathrm{~Hz}, 1 \mathrm{H}), 5.89$ (d, $J=10.1 \mathrm{~Hz}$, $1 \mathrm{H}), 6.11(\mathrm{dd}, J=10.4,1.5 \mathrm{~Hz}, 1 \mathrm{H}), 6.35(\mathrm{~d}, J=1.5 \mathrm{~Hz}, 1 \mathrm{H}), 7.31-7.43(\mathrm{~m}, 5 \mathrm{H}) ;{ }^{13} \mathrm{C} \mathrm{NMR}\left(\mathrm{CDCl}_{3}\right.$, 62.9 MHz) $\delta 136.5,132.2,131.4,130.3,128.5,128.4,126.3,118.9,108.4,96.9,78.1,71.0,51.1$, 50.9; EIMS $m / z$ (rel intensity) $354\left(\mathrm{M}^{+}, 3\right), 352\left(\mathrm{M}^{+}, 3\right), 273$ (4), 250 (20), 248 (23); HRMS (EIMS) calcd for $\mathrm{C}_{16} \mathrm{H}_{17} \mathrm{O}_{4} \mathrm{Br} 352.0310$ found 352.0304 .

9-Bromo-6,6-dimethoxy-(2R)-phenyl-1,4-dioxaspiro[4.(5R)]deca-7,9-diene $[(R R)-5]$. Anodic oxidation of a solution of the secondary alcohol $(R)-4(285 \mathrm{mg}, 0.9 \mathrm{mmol})$ in $\mathrm{MeOH}$ was performed according to the general procedure described above. Electrolysis was carried out at $100 \mathrm{~mA}$ by increasing potential from 1.40 to $2.40 \mathrm{~V} / \mathrm{Ag} / 0.1 \mathrm{M} \mathrm{AgCl}$. The reaction mixture was then processed as described above, and the residue was further dried overnight to give an oily crude product, which was purified by column chromatography, eluting with light petroleum/ $\mathrm{Et}_{2} \mathrm{O}(1: 1)$, to furnish the pure spiro-bisketal $(R R)-5$ as a yellow oil $(47 \mathrm{mg}, 15 \%):[\alpha]^{21}{ }_{\mathrm{D}}=-20.6^{\circ}\left(\mathrm{c}=1.40, \mathrm{CH}_{2} \mathrm{Cl}_{2}\right)$; IR ( $\mathrm{NaCl}) 2937,2848,1786,1651,1463,1384 \mathrm{~cm}^{-1} ;{ }^{1} \mathrm{H}$ NMR $\left(\mathrm{CDCl}_{3}, 300 \mathrm{MHz}\right) \delta 3.45$ (s, 3H), 3.51 $(\mathrm{s}, 3 \mathrm{H}), 3.71-3.89(\mathrm{~m}, 1 \mathrm{H}+$ impurities integrating for about $1 \mathrm{H}), 4.52(\mathrm{bt}, J=7.4 \mathrm{~Hz}, 1 \mathrm{H}), 5.25$ $(\mathrm{dd}, J=.8 .5,6.6 \mathrm{~Hz}, 1 \mathrm{H}), 5.91(\mathrm{~d}, J=10.2 \mathrm{~Hz}, 1 \mathrm{H}), 6.14(\mathrm{~d}, J=10.2 \mathrm{~Hz}, 1 \mathrm{H}), 6.27(\mathrm{~s}, 1 \mathrm{H}), 7.35$ 
(bs, 5H); ${ }^{13} \mathrm{C} \mathrm{NMR}\left(\mathrm{CDCl}_{3}, 75.5 \mathrm{MHz}\right) \delta 138.0,132.5,130.8,128.6,128.3,126.2,119.0,109.2$, 97.6, 79.0, 72.1, 51.4, 51.1; EIMS $m / z$ (rel intensity) $354\left(\mathrm{M}^{+}, 4\right), 352\left(\mathrm{M}^{+}, 4\right), 273$ (22), $250(20)$, 248 (20); HRMS (EIMS) calcd for $\mathrm{C}_{16} \mathrm{H}_{17} \mathrm{O}_{4} \mathrm{Br} 352.0310$ found 352.0300 .

9-Bromo-6,6-dimethoxy-(2S)-phenyl-1,4-dioxaspiro[4.(5S)]deca-7,9-diene $[(S S)-5]$. Anodic oxidation of a solution of the secondary alcohol $(S)-4(416 \mathrm{mg}, 1.3 \mathrm{mmol})$ in $\mathrm{MeOH}$ was performed according to the general procedure described. Electrolysis was carried out a $100 \mathrm{~mA}$ by increasing potential from 1.90 to $2.90 \mathrm{~V} / \mathrm{Ag} / 0.1 \mathrm{M} \mathrm{AgCl}$. The reaction mixture was then processed as described above, and the residue was further dried overnight to give an oily crude product, which was purified by column chromatography, eluting with light petroleum/ $\mathrm{Et}_{2} \mathrm{O}(4: 1 \rightarrow 3: 2)$, to furnish the pure spiro-bisketal $(S S)-5$ as a yellow oil $(65 \mathrm{mg}, 14 \%):[\alpha]^{21}{ }_{\mathrm{D}}=+31.5^{\circ}\left(\mathrm{c}=1.00, \mathrm{CH}_{2} \mathrm{Cl}_{2}\right)$; IR ( $\mathrm{NaCl}) 2937,2848,1786,1651,1463,1384 \mathrm{~cm}^{-1} ;{ }^{1} \mathrm{H}$ NMR $\left(\mathrm{CDCl}_{3}, 250 \mathrm{MHz}\right) \delta 3.45(\mathrm{~s}, 3 \mathrm{H}), 3.51$ (s, 3H), 3.76-3.88 (m, 1H 1H + impurities integrating for about $1 \mathrm{H}), 4.52(\mathrm{dd}, J=8.5,6.4 \mathrm{~Hz}, 1 \mathrm{H})$, $5.25(\mathrm{dd}, J=8.5,6.4 \mathrm{~Hz}, 1 \mathrm{H}), 5.90$ (d, $J=10.4 \mathrm{~Hz}, 1 \mathrm{H}), 6.14(\mathrm{dd}, J=10.4,1.5 \mathrm{~Hz}, 1 \mathrm{H}), 6.27$ (d, $J$ $=1.8 \mathrm{~Hz} 1 \mathrm{H}), 7.35(\mathrm{bs}, 5 \mathrm{H}) ;{ }^{13} \mathrm{C} \mathrm{NMR}\left(\mathrm{CDCl}_{3}, 75.5 \mathrm{MHz}\right) \delta 138.0,132.5,130.8,128.6,128.3$, 126.2, 119.0, 109.1, 97.5, 78.9, 72.1, 51.4, 51.1; EIMS $m / z$ (rel intensity) $354\left(\mathrm{M}^{+}, 0.5\right), 352$ $\left(\mathrm{M}^{+}, 0.6\right), 273$ (3), 250 (14), 248 (14); HRMS (EIMS) calcd for $\mathrm{C}_{16} \mathrm{H}_{17} \mathrm{O}_{4} \mathrm{Br} 352.0310$ found 352.0310 .

9-Bromo-(2R)-phenyl-1,4-dioxaspiro[4.(5S)]deca-7,9-dien-6-one [(RS)-6]. S e le c t i v e monohydrolysis of the spiro-bisketal $(R S)-5\left(37 \mathrm{mg}, 0.1 \mathrm{mmol}^{2}\right)$ in $^{\mathrm{Et}} \mathrm{E}_{2} \mathrm{O}(5 \mathrm{~mL})$ was carried out at $0^{\circ} \mathrm{C}$ by treatment with TFA $(3 \mathrm{~mL})$ and water $(1 \mathrm{~mL})$. The reaction mixture was stirred for $1 \mathrm{~h}$, after which time it was neutralized with $\mathrm{NaHCO}_{3}(3 \times 10 \mathrm{~mL})$, dried over $\mathrm{Na}_{2} \mathrm{SO}_{4}$, filtered, evaporated and submitted to column chromatography, eluting with $\mathrm{CH}_{2} \mathrm{Cl}_{2}$, to give the orthoquinone spiromonoketal $(R S)-6$ as a yellow oil $(17 \mathrm{mg}, 53 \%):[\alpha]^{21}{ }_{\mathrm{D}}+6.8^{\circ}\left(\mathrm{c}=0.43, \mathrm{CHCl}_{3}\right)$; IR $(\mathrm{NaCl}) 2926$, 1740, 1659, $1636 \mathrm{~cm}^{-1} ;{ }^{1} \mathrm{H}$ NMR $\left(\mathrm{CDCl}_{3}, 250 \mathrm{MHz}\right) \delta 4.21$ (bt, $\left.J=8.9 \mathrm{~Hz}, 1 \mathrm{H}\right), 4.43$ (dd, $J=8.1$, $5.7 \mathrm{~Hz}, 1 \mathrm{H}), 5.17$ (dd, $J=9.8,5.5 \mathrm{~Hz}, 1 \mathrm{H}), 6.00$ (d, $J=10.4 \mathrm{~Hz}, 1 \mathrm{H}), 6.55$ (d, $J=1.9 \mathrm{~Hz}, 1 \mathrm{H}), 6.92$ $(\mathrm{dd}, J=10.2,2.0 \mathrm{~Hz}, 1 \mathrm{H}), 7.37-7.53(\mathrm{~m}, 5 \mathrm{H}) ;{ }^{13} \mathrm{C} \mathrm{NMR}\left(\mathrm{CDCl}_{3}, 50.3 \mathrm{MHz}\right) \delta 196.1,143.8,137.1$, 
135.4, 128.8, 128.6, 127.4, 125.5, 119.9, 97.8, 80.1, 72.7; MALDI $m / z 306\left(\mathrm{M}^{+}\right), 308\left(\mathrm{M}^{+}\right)$; HRMS (MALDI) calcd for $\mathrm{C}_{14} \mathrm{H}_{11} \mathrm{O}_{3} \mathrm{Br} 306.9969$ found 306.9835.

9-Bromo-(2S)-phenyl-1,4-dioxaspiro[4.(5R)]deca-7,9-dien-6-one $\quad[(S \boldsymbol{R})-6]$. S e le c t i v e monohydrolysis of the spiro-bisketal $(S R)-5(82 \mathrm{mg}, 0.2 \mathrm{mmol})$ in $\mathrm{Et}_{2} \mathrm{O}(10 \mathrm{~mL})$ was carried out at $0^{\circ} \mathrm{C}$ by treatment with TFA $(6 \mathrm{~mL})$ and water $(2 \mathrm{~mL})$. The reaction mixture was stirred for $1 \mathrm{~h}$, after which time it was neutralized with $\mathrm{NaHCO}_{3}(3 \times 10 \mathrm{~mL})$, dried over $\mathrm{Na}_{2} \mathrm{SO}_{4}$, filtered, evaporated and submitted to column chromatography, eluting with $\mathrm{CH}_{2} \mathrm{Cl}_{2}$, to give the orthoquinone spiromonoketal $(S R)-6$ as a yellow oil $(41 \mathrm{mg}, 60 \%):[\alpha]^{21}{ }_{\mathrm{D}}-7.6^{\circ}\left(\mathrm{c}=0.52, \mathrm{CHCl}_{3}\right) ; \mathrm{IR}(\mathrm{NaCl}) 2927$, 2848, 1792, 1695, $1628 \mathrm{~cm}^{-1} ;{ }^{1} \mathrm{H}$ NMR $\left(\mathrm{CDCl}_{3}, 250 \mathrm{MHz}\right) \delta 4.20,(\mathrm{bt}, J=9.0 \mathrm{~Hz}, 1 \mathrm{H}), 4.43$ (dd, $J$ $=8.2,5.5 \mathrm{~Hz}, 1 \mathrm{H}), 5.16(\mathrm{dd}, J=9.8,5.8 \mathrm{~Hz}, 1 \mathrm{H}), 6.00(\mathrm{~d}, J=10.4 \mathrm{~Hz}, 1 \mathrm{H}), 6.55(\mathrm{~d}, J=2.4 \mathrm{~Hz}$, $1 \mathrm{H}), 6.92(\mathrm{dd}, J=10.4,2.4 \mathrm{~Hz}, 1 \mathrm{H}), 7.36-7.53(\mathrm{~m}, 5 \mathrm{H}) ;{ }^{13} \mathrm{C} \mathrm{NMR}\left(\mathrm{CDCl}_{3}, 62.9 \mathrm{MHz}\right) \delta 196.1$, 143.8, 137.1, 135.4, 128.8, 128.6, 127.4, 125.5, 119.9, 97.8, 80.1, 72.7; MALDI m/z $306\left(\mathrm{M}^{+}\right), 308$ $\left(\mathrm{M}^{+}\right)$; HRMS (MALDI) calcd for $\mathrm{C}_{14} \mathrm{H}_{11} \mathrm{O}_{3} \mathrm{Br} 306.9969$ found 306.9776.

9-Bromo-(2R)-phenyl-1,4-dioxaspiro[4.(5R)]deca-7,9-dien-6-one $[(\boldsymbol{R} R)-6]$. S e lective monohydrolysis of the spiro-bisketal $(R R)-5(94 \mathrm{mg}, 0.3 \mathrm{mmol})$ in $\mathrm{Et}_{2} \mathrm{O}(10 \mathrm{~mL})$ was carried out at $0^{\circ} \mathrm{C}$ by treatment with TFA $(6 \mathrm{~mL})$ and water $(2 \mathrm{~mL})$. The reaction mixture was stirred for $1 \mathrm{~h}$, after which time it was neutralized with $\mathrm{NaHCO}_{3}(3 \times 10 \mathrm{~mL})$, dried over $\mathrm{Na}_{2} \mathrm{SO}_{4}$, filtered, evaporated and submitted to column chromatography, eluting with $\mathrm{CH}_{2} \mathrm{Cl}_{2}$, to give the orthoquinone spiromonoketal $(R R)-6$ as a yellow oil (44 mg, 49\%): $[\alpha]^{21}{ }_{\mathrm{D}}-14.2^{\circ}\left(\mathrm{c}=1.09, \mathrm{CHCl}_{3}\right)$; IR $(\mathrm{NaCl}) 2929$, 2852, 1731, 1681, $1652 \mathrm{~cm}^{-1} ;{ }^{1} \mathrm{H} \mathrm{NMR}\left(\mathrm{CDCl}_{3}, 250 \mathrm{MHz}\right) \delta 3.83-3.96(\mathrm{~m}, 1 \mathrm{H}+$ impurities integrating for about $1 H), 4.64(\mathrm{bt}, J=7.2 \mathrm{~Hz}, 1 \mathrm{H}), 5.58(\mathrm{bt}, J=6.9 \mathrm{~Hz}, 1 \mathrm{H}), 5.97(\mathrm{~d}, J=10.4 \mathrm{~Hz}$, $1 \mathrm{H}), 6.58(\mathrm{~d}, J=2.1 \mathrm{~Hz}, 1 \mathrm{H}), 6.90(\mathrm{dd}, J=10.2,2.3 \mathrm{~Hz}, 1 \mathrm{H}), 7.37(\mathrm{bs}, 5 \mathrm{H}) ;{ }^{13} \mathrm{C} \mathrm{NMR}\left(\mathrm{CDCl}_{3}, 62.9\right.$ $\mathrm{MHz}) \delta 195.9,143.7,137.6,136.3,128.7,128.6,126.4,125.5,120.7,98.7,79.1,72.3$; APCIMS/MS m/z 309 (MH+, 100), $307\left(\mathrm{MH}^{+}, 96\right), 227$ (13), 189 (92), 187 (100); HRMS (MALDI) calcd for $\mathrm{C}_{14} \mathrm{H}_{11} \mathrm{O}_{3} \mathrm{Br} 306.9969$ found 306.9817 . 
9-Bromo-(2S)-phenyl-1,4-dioxaspiro[4.(5S)]deca-7,9-dien-6-one [(SS)-6]. S e le c t i v e monohydrolysis of the spiro-bisketal $(S S)-5(65 \mathrm{mg}, 0.2 \mathrm{mmol})$ in $^{\mathrm{Et}_{2} \mathrm{O}}(10 \mathrm{~mL})$ was carried out at $0^{\circ} \mathrm{C}$ by treatment with TFA $(6 \mathrm{~mL})$ and water $(2 \mathrm{~mL})$. The reaction mixture was stirred for $1 \mathrm{~h}$, after which time it was neutralized with $\mathrm{NaHCO}_{3}(3 \times 10 \mathrm{~mL})$, dried over $\mathrm{Na}_{2} \mathrm{SO}_{4}$, filtered, evaporated and submitted to column chromatography, eluting with $\mathrm{CH}_{2} \mathrm{Cl}_{2}$, to give the orthoquinone spiromonoketal $(S S)-6$ as a yellow oil $(25 \mathrm{mg}, 43 \%):[\alpha]^{21}{ }_{\mathrm{D}}+7.9^{\circ}\left(\mathrm{c}=1.23, \mathrm{CHCl}_{3}\right) ; \mathrm{IR}(\mathrm{NaCl}) 2929$, 2852, 1731, 1681, $1652 \mathrm{~cm}^{-1} ;{ }^{1} \mathrm{H}$ NMR $\left(\mathrm{CDCl}_{3}, 250 \mathrm{MHz}\right) \delta 3.83-3.96(\mathrm{~m}, 1 \mathrm{H}+$ impurities integrating for about 1H), 4.64 (bt, $J=7.3 \mathrm{~Hz}, 1 \mathrm{H}), 5.58$ (bt, $J=6.7 \mathrm{~Hz}, 1 \mathrm{H}), 5.97(\mathrm{~d}, J=10.1 \mathrm{~Hz}$, $1 \mathrm{H}), 6.58(\mathrm{~s}, 1 \mathrm{H}), 6.90(\mathrm{dd}, J=10.4,2.1 \mathrm{~Hz}, 1 \mathrm{H}), 7.37(\mathrm{bs}, 5 \mathrm{H}) ;{ }^{13} \mathrm{C} \mathrm{NMR}\left(\mathrm{CDCl}_{3}, 50.3 \mathrm{MHz}\right) \delta$ $195.9,143.7,137.7,136.4,128.7,128.6,126.4,125.6,120.7,98.8,79.1,72.4$; MALDI $m / z 306$ $\left(\mathrm{M}^{+}\right), 308\left(\mathrm{M}^{+}\right)$; HRMS (MALDI) calcd for $\mathrm{C}_{14} \mathrm{H}_{11} \mathrm{O}_{3} \mathrm{Br} 306.9969$ found 306.9751.

NB: The four enantiopure orthoquinone spiro-monoketals described above are stable in $0.02 \mathrm{M}$ solution in $\mathrm{Et}_{2} \mathrm{O}$. Their 4-bromine substituent do retard dimerization, but they still tend to dimerize at room temperature upon standing as oily residues and in concentrated solutions. The fact that no or very little racemization occurred at the spiro-center during monohydrolysis was clearly evidenced by ${ }^{1} \mathrm{H}$ NMR analysis, since the diastereoisomers $(R R)$ - and $(R S)-\mathbf{6}$, as well as their enantiomeric counterparts, display different ${ }^{1} \mathrm{H}$ NMR characteristics. Their optical rotations are in agreement with this analysis (vide supra). Examination of further synthetic transformations of these enantiopure orthoquinone monoketals was performed only using the (RS)-6 enantiomer (vide infra).

Cyclohexa-2,4-dienols 7. $\mathrm{LiAlH}_{4}(17 \mathrm{mg}, 0.5 \mathrm{mmol})$ and $\mathrm{LiBr}(50 \mathrm{mg}, 0.6 \mathrm{mmol})$ were dried beforehand at $140^{\circ} \mathrm{C}$ for $3 \mathrm{~h}$ under reduced pressure. ${ }^{[3]}$ After cooling, a solution of $(R S)-6$ (26 mg, $0.09 \mathrm{mmol})$ in anhydrous $\mathrm{Et}_{2} \mathrm{O}(5 \mathrm{~mL})$ was then added and the resulting mixture was stirred at $-78^{\circ} \mathrm{C}$ for $1 \mathrm{~h}$, after which time the excess reducing agent was quenched with saturated aqueous $\mathrm{NH}_{4} \mathrm{Cl}$. The mixture was diluted in $\mathrm{H}_{2} \mathrm{O}(5 \mathrm{~mL})$, separated and the aqueous layer was extracted with $\mathrm{Et}_{2} \mathrm{O}(3$ $\times 10 \mathrm{~mL}$ ). The combined organic layers were washed with brine, dried over $\mathrm{Na}_{2} \mathrm{SO}_{4}$, filtered and evaporated to give a residue, which was submitted to column chromatography, eluting with light petroluem/Et ${ }_{2} \mathrm{O}(1: 1)$, to afford 7 as diastereoisomeric mixture $(10 \mathrm{mg}, 38 \%)$ in $c a$. 30\% de: IR 
( $\mathrm{NaCl}) 3397,2926,1490,1444 \mathrm{~cm}^{-1}$; diastereoisomer I: ${ }^{1} \mathrm{H}$ NMR $\left(\mathrm{CDCl}_{3}, 250 \mathrm{MHz}\right) \delta 3.79-4.05$ $(\mathrm{m}, 3 \mathrm{H}), 5.04(\mathrm{dd}, J=7.6,3.4 \mathrm{~Hz}, 1 \mathrm{H}), 6.79-7.04(\mathrm{~m}, 3 \mathrm{H}), 7.38-7.40(\mathrm{~m}, 5 \mathrm{H}) ;{ }^{13} \mathrm{C} \mathrm{NMR}\left(\mathrm{CDCl}_{3}\right.$, 62.9 MHz) $\delta 145.9,136.7,128.9,128.8,126.3,126.2,120.5,117.2,110.9,84.5,77.2,66.9$; EIMS $m / z$ (rel intensity) $310\left(\mathrm{M}^{+}, 5\right), 308\left(\mathrm{M}^{+}, 5\right), 190$ (79), 188 (82), 121 (100); diastereoisomer II: ${ }^{1} \mathrm{H}$ NMR $\left(\mathrm{CDCl}_{3}, 250 \mathrm{MHz}\right) \delta$ 4.09-4.18 (m, 3H), $5.13(\mathrm{dd}, J=8.4,3.2 \mathrm{~Hz}, 1 \mathrm{H}), 6.79-7.04(\mathrm{~m}, 3 \mathrm{H})$, 7.38-7.40 (m, 5H); ${ }^{13} \mathrm{C} \mathrm{NMR}\left(\mathrm{CDCl}_{3}, 62.9 \mathrm{MHz}\right) \delta 146.5,139.2,129.0,128.7,126.3,126.2,125.5$, 116.9, 111.2, 84.5, 74.8, 72.9; EIMS $m / z$ (rel intensity) $310\left(\mathrm{M}^{+}, 5\right), 308\left(\mathrm{M}^{+}, 5\right), 190(79), 188$ (82), 121 (100); HRMS (EIMS) calcd for $\mathrm{C}_{14} \mathrm{H}_{13} \mathrm{O}_{3} \mathrm{Br} 308.0048$ found 308.0047.

Cyclohex-3-enone 8. A 1.0 M solution of L-selectride in THF (780 $\mu \mathrm{L}, 0.8 \mathrm{mmol})$ was added dropwise at $-78^{\circ} \mathrm{C}$ to a solution of $(R S)-6(95 \mathrm{mg}, 0.3 \mathrm{mmol})$ in anhydrous THF $(3.5 \mathrm{~mL})$. The resulting mixture was stirred $-78^{\circ} \mathrm{C}$ for $1 \mathrm{~h}$, after which time the excess reducing agent was quenched with a aqueous $10 \% \mathrm{NaOH}(8 \mathrm{~mL})$. The mixture was extracted with EtOAc $(3 \times 5 \mathrm{~mL})$ and the combined organic layers were washed with brine, dried over $\mathrm{Na}_{2} \mathrm{SO}_{4}$, filtered and evaporated to give a residue, which was submitted to column chromatography, eluting with light petroluem/Et ${ }_{2} \mathrm{O}(1: 1)$, to afford $8(32 \mathrm{mg}, 33 \%)$. $[\alpha]^{21}{ }_{\mathrm{D}}-8.2^{\circ}\left(\mathrm{c}=1.08, \mathrm{CHCl}_{3}\right)$; IR $(\mathrm{NaCl}) 2896$, 1746, 1646, $1456 \mathrm{~cm}^{-1} ;{ }^{1} \mathrm{H}$ NMR $\left(\mathrm{CDCl}_{3}, 250 \mathrm{MHz}\right) \delta 2.80-3.00$ (m, 4H), 3.90 (bt, $\left.J=9.2 \mathrm{~Hz}, 1 \mathrm{H}\right)$, $4.42(\mathrm{dd}, J=8.4,5.6 \mathrm{~Hz}, 1 \mathrm{H}), 5.11(\mathrm{dd}, J=9.6,5.6 \mathrm{~Hz}, 1 \mathrm{H}), 6.19(\mathrm{~s}, 1 \mathrm{H}), 7.34-7.40(\mathrm{~m}, 5 \mathrm{H}) ;{ }^{13} \mathrm{C}$ $\mathrm{NMR}\left(\mathrm{CDCl}_{3}, 62.9 \mathrm{MHz}\right) \delta 202.1,136.2,130.3,130.1,128.7,128.6,126.9,101.3,79.8,73.1,37.1$, 35.7; EIMS m/z (rel intensity) $310\left(\mathrm{M}^{+}, 1\right), 308\left(\mathrm{M}^{+}, 1\right), 282$ (2), 280 (2), 229 (3), 201 (100).

1-phenylcyclohexa-2,4-diene-1-ol 9. A 1.0 M solution of PhMgBr in THF (550 $\mu \mathrm{L}, 0.6 \mathrm{mmol}$ ) was added at $-78^{\circ} \mathrm{C}$ to a solution of $(R S)-6(68 \mathrm{mg}, 0.2 \mathrm{mmol})$ in anhydrous THF $(5 \mathrm{~mL})$. The resulting mixture was stirred at $-78^{\circ} \mathrm{C}$ for $1 \mathrm{~h}$, after which time the excess Grignard reagent was quenched with saturated aqueous $\mathrm{NaHCO}_{3}$. The mixture was diluted in $\mathrm{H}_{2} \mathrm{O}(5 \mathrm{~mL})$, separated and the aqueous layer was extracted with $\mathrm{Et}_{2} \mathrm{O}(3 \times 10 \mathrm{~mL})$. The combined organic layers were washed with brine, dried over $\mathrm{Na}_{2} \mathrm{SO}_{4}$, filtered and evaporated to give a residue, which was submitted to column chromatography, eluting with light petroluem/ $\mathrm{Et}_{2} \mathrm{O}(1: 1)$, to afford 9 (74 $\left.\mathrm{mg}, 88 \%\right)$ in $90 \%$ de. Major diastereoisomer only: $[\alpha]^{21}{ }_{\mathrm{D}}+83.1^{\circ}\left(\mathrm{c}=1.29, \mathrm{CHCl}_{3}\right)$; IR $(\mathrm{NaCl}) 3450,2930,2848,1742$, 
1496, $1450 \mathrm{~cm}^{-1}$; ${ }^{1} \mathrm{H}$ NMR $\left(\mathrm{CDCl}_{3}, 200 \mathrm{MHz}\right) \delta 3.40$ (s, 1H), 3.59 (bt, $\left.J=8.4 \mathrm{~Hz}, 1 \mathrm{H}\right), 4.23$ (dd, $J$ $=8.1,6.2 \mathrm{~Hz}, 1 \mathrm{H}), 5.14(\mathrm{dd}, J=8.7,6.2 \mathrm{~Hz}, 1 \mathrm{H}), 5.93(\mathrm{~d}, J=10.0 \mathrm{~Hz}, 1 \mathrm{H}), 6.15(\mathrm{dd}, J=10.0,1.8$ $\mathrm{Hz}, 1 \mathrm{H}), 6.23(\mathrm{~d}, J=1.6 \mathrm{~Hz}, 1 \mathrm{H}), 7.39-7.66(\mathrm{~m}, 10 \mathrm{H}) ;{ }^{13} \mathrm{C} \mathrm{NMR}\left(\mathrm{CDCl}_{3}, 50.3 \mathrm{MHz}\right) \delta 138.3$, 137.2, 135.9, 129.2, 129.0, 128.9, 128.1, 127.9, 127.4, 127.1, 126.7, 121.0, 109.8, 78.5, 71.3; EIMS $m / z$ (rel intensity) $386\left(\mathrm{M}^{+}, 0.2\right), 384\left(\mathrm{M}^{+}, 0.2\right), 368$ (0.5), 366 (0.5), 305 (2), 282 (4), 280 (4); HRMS (EIMS) calcd for $\mathrm{C}_{20} \mathrm{H}_{17} \mathrm{O}_{3} \mathrm{Br} 384.0361$ found 384.0355 .

\section{2-(4-Allyl-5-bromo-2-hydroxyphenoxy)-(2R)-phenylethanol (10a) and 2-(4-allyl-5-bromo-2-} hydroxyphenoxy)-(1R)-phenylethanol (10b). A $1.0 \mathrm{M}$ solution of allylmagnesium bromide in ether $(2.2 \mathrm{~mL}, 2.2 \mathrm{mmol})$ was added at $-78^{\circ} \mathrm{C}$ to a solution of $(R S)-6(68 \mathrm{mg}, 0.2 \mathrm{mmol})$ in anhydrous THF $(5 \mathrm{~mL})$. The resulting mixture was stirred at $-78^{\circ} \mathrm{C}$ for $1 \mathrm{~h}$, after which time the excess Grignard reagent was quenched with saturated aqueous $\mathrm{NaHCO}_{3}$. The mixture was diluted in $\mathrm{H}_{2} \mathrm{O}(5 \mathrm{~mL})$, separated and the aqueous layer was extracted with $\mathrm{Et}_{2} \mathrm{O}(3 \times 10 \mathrm{~mL})$. The combined organic layers were washed with brine, dried over $\mathrm{Na}_{2} \mathrm{SO}_{4}$, filtered and evaporated to give a residue, which was submitted to column chromatography, eluting with light petroluem/ $\operatorname{Et}_{2} \mathrm{O}(1: 1)$, to afford 10a (22 mg, 29\%) and 10b (25 mg, 32\%) as yellow oils. 10a: IR (NaCl) 3340, 2928, $1490 \mathrm{~cm}^{-1} ;{ }^{1} \mathrm{H}$ NMR $\left(\mathrm{CDCl}_{3}, 300 \mathrm{MHz}\right) \delta 3.27(\mathrm{~d}, J=6.4 \mathrm{~Hz}, 2 \mathrm{H}), 3.80-3.93(\mathrm{~m}, 2 \mathrm{H}), 4.93-5.02$ (m, 3H), 5.75$5.88(\mathrm{~m}, 1 \mathrm{H}), 6.76(\mathrm{~s}, 2 \mathrm{H}), 7.19-7.32(\mathrm{~m}, 5 \mathrm{H}) ;{ }^{13} \mathrm{C} \mathrm{NMR}\left(\mathrm{CDCl}_{3}, 75.5 \mathrm{MHz}\right) \delta 147.0,144.4,136.8$, $135.6,134.5,128.9,128.7,126.3,121.4,117.4,116.4,112.6,84.6,67.0,39.6$; EIMS $\mathrm{m} / z$ (rel intensity) $350\left(\mathrm{M}^{+}, 1\right), 348\left(\mathrm{M}^{+}, 1\right), 332$ (2), 330 (2), 230 (49), 228 (54). 10b: IR (NaCl) 3340, 2928, $1490 \mathrm{~cm}^{-1} ;{ }^{1} \mathrm{H}$ NMR $\left(\mathrm{CDCl}_{3}, 300 \mathrm{MHz}\right) \delta 3.39$ (d, $\left.J=6.03 \mathrm{~Hz}, 2 \mathrm{H}\right), 3.83-4.15$ (m, 2H), 5.04$5.13(\mathrm{~m}, 3 \mathrm{H}), 5.85-5.98(\mathrm{~m}, 1 \mathrm{H}), 6.84(\mathrm{~s}, 1 \mathrm{H}), 7.03(\mathrm{~s}, 1 \mathrm{H}), 7.38-7.41(\mathrm{~m}, 5 \mathrm{H}) ;{ }^{13} \mathrm{C} \mathrm{NMR}\left(\mathrm{CDCl}_{3}\right.$, $62.9 \mathrm{MHz}) \delta 146.1,144.8,139.2,135.7,133.7,128.8,128.7,126.2,118.5,117.2,116.4,112.9$, 75.2, 72.9, 39.6; EIMS $m / z$ (rel intensity) $350\left(\mathrm{M}^{+}, 11\right), 348\left(\mathrm{M}^{+}, 12\right), 332$ (27), 330 (29), 230 (65), $228(70)$.

Cyclohex-3-enone 11. To a $-50^{\circ} \mathrm{C}$ cooled solution of dimethylmalonate ( $\left.30 \mu \mathrm{L}, 0.25 \mathrm{mmol}\right)$ and KHMDS (43 mg, $0.21 \mathrm{mmol})$ in anhydrous THF $(2 \mathrm{~mL})$ was added $(R S)-6(57 \mathrm{mg}, 0.2 \mathrm{mmol})$ in anhydrous THF $(3 \mathrm{~mL})$. The resulting mixture was stirred $-50^{\circ} \mathrm{C}$ for 1 hour, after which time it was 
diluted in $\mathrm{Et}_{2} \mathrm{O}(10 \mathrm{~mL})$ and $\mathrm{H}_{2} \mathrm{O}(5 \mathrm{~mL})$. After separation, the aqueous phase was extracted with $\mathrm{Et}_{2} \mathrm{O}(2 \times 10 \mathrm{~mL})$, acidified with aqueous $\mathrm{HCl} 10 \%$ and further extracted with $\mathrm{Et}_{2} \mathrm{O}(2 \times 10 \mathrm{~mL})$. The combined organic layers were washed with brine, dried over $\mathrm{Na}_{2} \mathrm{SO}_{4}$, filtered and evaporated to give a residue, which was submitted to column chromatography, eluting with light petroluem/Et ${ }_{2} \mathrm{O}$ (1:1), to afford 11 as a diastereoisomeric mixture (28 mg, 34\%) in 30\% de: IR (NaCl) 2958, 2900, 1748, 1502, $1444 \mathrm{~cm}^{-1} ;{ }^{1} \mathrm{H} \mathrm{NMR}\left(\mathrm{CDCl}_{3}, 250 \mathrm{MHz}\right) \delta 3.07$ (bt, $\left.J=5.95 \mathrm{~Hz}, 2 \mathrm{H}\right), 3.63-3.87$ (m, $8 \mathrm{H}), 4.11(\mathrm{bt}, J=3.51 \mathrm{~Hz}, 1 \mathrm{H}), 4.35(\mathrm{dd}, J=8.4,5.6 \mathrm{~Hz}, 1 \mathrm{H}), 4.46(\mathrm{dd}, J=8.2,5.8 \mathrm{~Hz}, 1 \mathrm{H}), 5.10$ $(\mathrm{dd}, J=9.8,5.8 \mathrm{~Hz}, 1 \mathrm{H}), 6.31(\mathrm{~d}, J=3.35 \mathrm{~Hz}, 1 \mathrm{H}), 7.36-7.58(\mathrm{~m}, 5 \mathrm{H}) ;{ }^{13} \mathrm{C} \mathrm{NMR}\left(\mathrm{CDCl}_{3}, 75.5\right.$ MHz) $\delta 200.8,200.2,167.9,166.9,136.2$, 136.0, 133.0, 132.8, 130.5, 130.3, 128.7, 128.6, 128.5, $127.1,127.0,100.2,99.8,80.3,79.9,73.9,72.7,56.1,53.0,52.7,52.6,52.5,44.6,41.1,39.7,39.5$; EIMS $m / z$ (rel intensity) $440\left(\mathrm{M}^{+}, 0.02\right), 438\left(\mathrm{M}^{+}, 0.02\right), 359$ (12), 331 (29); HRMS (EIMS) calcd for $\mathrm{C}_{19} \mathrm{H}_{19} \mathrm{O}_{7} \mathrm{Br} 438.0314$ found 438.022 .

\section{Additional References}

[1] A. I. Meyers, L. Snyder, J. Org. Chem. 1993, 58, 36.

[2] M. Van der Mey, A. Hatzelmann, G. P. M. Van Klink, I. J. Van der Laan, G. J. Sterk, U. Thibaut, W. R. Ulrich, H. Timmerman, J. Med. Chem. 2001, 44, 2523.

[3] H. Fujioka, H. Annoura, K. Murano, Y. Kita, Y. Tamura, Chem. Pharm. Bull. 1989, 37, 2047. 


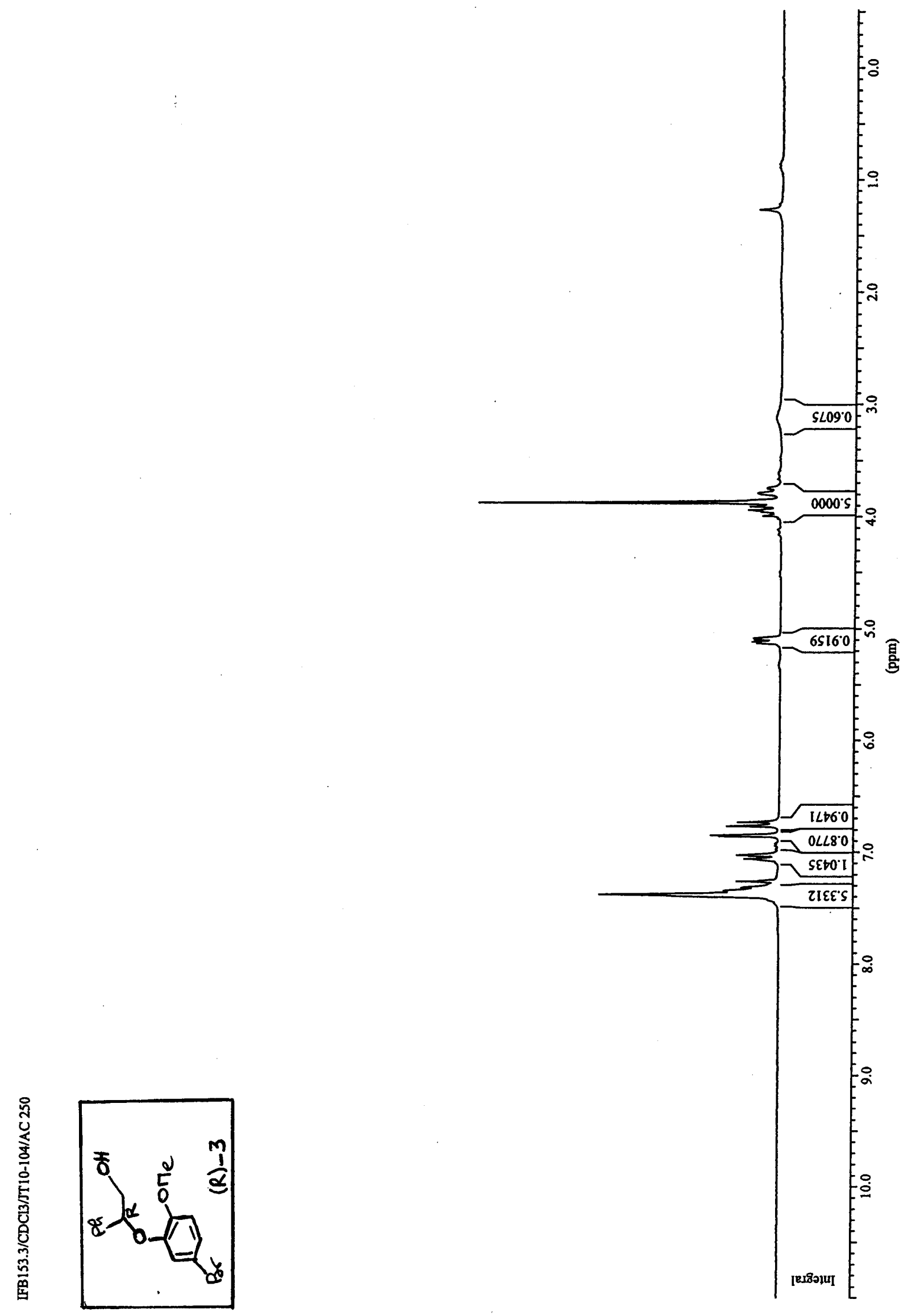


File Name

Peak Results saved in File:

Nucleus

$\mathrm{SF}$

OFFSET

SW_p

Peak Picking Parameter

Peak constant $\mathrm{PC}=$

Noise

Sens. level
1.00

15

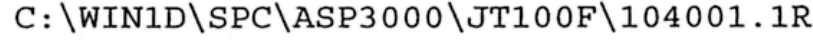

$1 \mathrm{H}$

$250.132854 \mathrm{MHz}$

$15.7760 \mathrm{ppm}$

$5000.00 \mathrm{~Hz}$

16384

\section{Peak Picking region}

\begin{tabular}{ccrr} 
Start $(\mathrm{ppm} / \mathrm{Hz})$ & End $(\mathrm{ppm} / \mathrm{Hz})$ & MI $(\%)$ & MAXI (\%) \\
\hline $7.46 / 1867.2$ & $7.30 / 1825.4$ & 10.86 & 62.88 \\
$7.29 / 1823.0$ & $7.20 / 1800.7$ & 11.12 & 20.19 \\
$7.09 / 1774.5$ & $7.00 / 1750.4$ & 6.40 & 19.14 \\
$6.88 / 1720.1$ & $6.81 / 1704.3$ & 17.56 & 27.02 \\
$6.78 / 1695.1$ & $6.69 / 1672.2$ & 11.26 & 20.06 \\
$5.18 / 1295.6$ & $4.97 / 1244.4$ & 4.55 & 12.79 \\
$4.05 / 1012.7$ & $3.49 / 873.0$ & 81.08 & 117.70 \\
$4.08 / 1021.6$ & $3.62 / 905.6$ & 4.01 & 20.32 \\
$3.17 / 793.0$ & $3.05 / 763.4$ & -0.83 & 8.32 \\
\hline
\end{tabular}

Peak Picking results

\begin{tabular}{rrrrrr} 
Peak Nr. Data Point & Frequency & \multicolumn{1}{c}{ PPM } & Intensity & \% Int. \\
\hline 1 & 6874 & 1848.31 & 7.3893 & 4029 & 35.9 \\
2 & 6886 & 1844.65 & 7.3747 & 6618 & 58.9 \\
3 & 6905 & 1838.85 & 7.3515 & 2011 & 17.9 \\
4 & 6915 & 1835.80 & 7.3393 & 2024 & 18.0 \\
5 & 6934 & 1830.00 & 7.3161 & 1385 & 12.3 \\
6 & 6980 & 1815.96 & 7.2600 & 1562 & 13.9 \\
7 & 7140 & 1767.14 & 7.0648 & 969 & 8.6 \\
8 & 7147 & 1765.00 & 7.0563 & 1278 & 11.4 \\
9 & 7168 & 1758.59 & 7.0306 & 1123 & 10.0 \\
10 & 7176 & 1756.15 & 7.0209 & 1549 & 13.8 \\
11 & 7313 & 1714.34 & 6.8537 & 2195 & 19.6 \\
12 & 7320 & 1712.20 & 6.8452 & 2517 & 22.4 \\
13 & 7386 & 1692.06 & 6.7647 & 1924 & 17.1 \\
14 & 7414 & 1683.52 & 6.7305 & 1538 & 13.7 \\
15 & 8729 & 1282.21 & 5.1261 & 876 & 7.8 \\
16 & 8740 & 1278.86 & 5.1127 & 1051 & 9.4 \\
17 & 8757 & 1273.67 & 5.0920 & 964 & 8.6 \\
18 & 8768 & 1270.31 & 5.0785 & 989 & 8.8 \\
19 & 9662 & 997.48 & 3.9878 & 701 & 6.2 \\
20 & 9691 & 988.63 & 3.9524 & 771 & 6.9 \\
21 & 9702 & 985.28 & 3.9390 & 1216 & 10.8 \\
22 & 9730 & 976.73 & 3.9048 & 1152 & 10.3 \\
23 & 9759 & 967.88 & 3.8695 & 11229 & 100.0 \\
24 & 9776 & 962.69 & 3.8487 & 1407 & 12.5 \\
25 & 9828 & 946.82 & 3.7853 & 893 & 7.9 \\
26 & 9860 & 937.06 & 3.7462 & 529 & 4.7 \\
27 & 10379 & 778.67 & 3.1130 & 198 & 1.8 \\
\hline
\end{tabular}



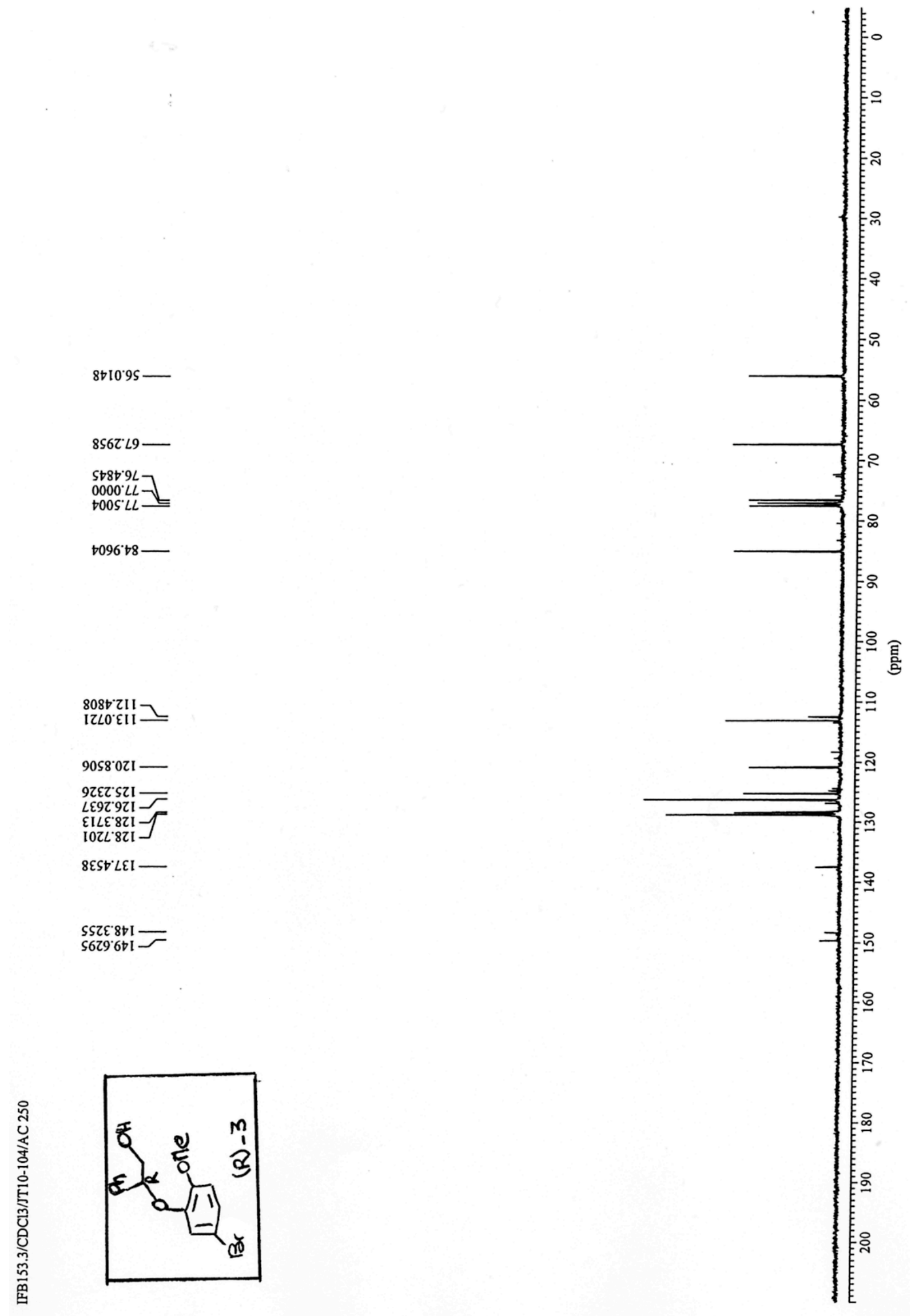


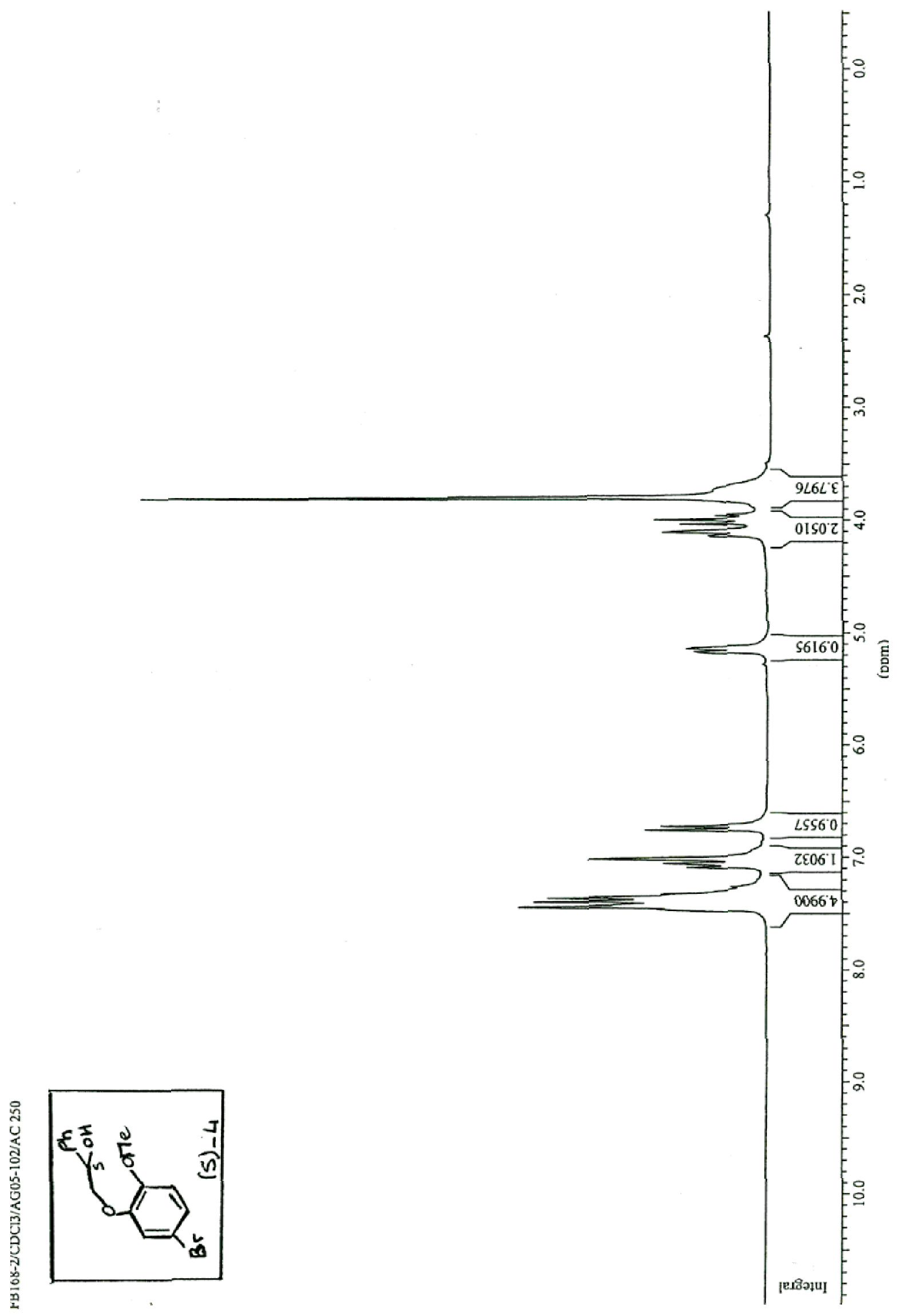


File Name

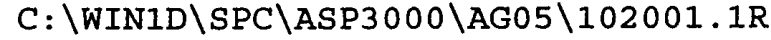

Peak Results saved in File :

Nucleus

SF

OFFSET

SW_P

: $1 \mathrm{H}$

: $250.132854 \mathrm{MHz}$

: $15.7772 \mathrm{ppm}$

: $5000.00 \mathrm{~Hz}$

: 16384

Peak Picking Parameter

Peak constant $\mathrm{PC}=$

Noise

1.00

Sens. level =

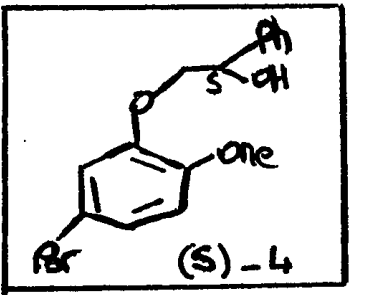

Peak Picking region

\begin{tabular}{rrrr} 
Start $(\mathrm{ppm} / \mathrm{Hz})$ & End $(\mathrm{ppm} / \mathrm{Hz})$ & MI $(8)$ & MAXI $(q)$ \\
\hline $7.62 / 1906.6$ & $6.58 / 1646.0$ & 4.44 & 40.21 \\
$5.20 / 1300.5$ & $5.07 / 1269.4$ & 8.05 & 14.04 \\
$4.28 / 1071.3$ & $3.88 / 971.5$ & 4.44 & 18.36 \\
$3.93 / 982.2$ & $3.57 / 893.7$ & 76.37 & 117.67 \\
\hline
\end{tabular}

\section{Peak Picking results}

\begin{tabular}{rrrrrr} 
Peak Nr. Data Point & Frequency & \multicolumn{1}{c}{ PPM } & Intensity & o Int. \\
\hline 1 & 6818 & 1865.71 & 7.4589 & 493 & 15.6 \\
2 & 6841 & 1858.69 & 7.4308 & 1111 & 35.2 \\
3 & 6855 & 1854.42 & 7.4137 & 594 & 18.8 \\
4 & 6860 & 1852.89 & 7.4076 & 581 & 18.4 \\
5 & 6881 & 1846.48 & 7.3820 & 1070 & 33.9 \\
6 & 6906 & 1838.85 & 7.3515 & 956 & 30.3 \\
7 & 6932 & 1830.92 & 7.3198 & 433 & 13.7 \\
8 & 6981 & 1815.96 & 7.2600 & 146 & 4.6 \\
9 & 7124 & 1772.32 & 7.0855 & 329 & 10.4 \\
10 & 7131 & 1770.19 & 7.0770 & 365 & 11.5 \\
11 & 7152 & 1763.78 & 7.0514 & 416 & 13.2 \\
12 & 7159 & 1761.64 & 7.0428 & 473 & 15.0 \\
13 & 7189 & 1752.49 & 7.0062 & 856 & 27.1 \\
14 & 7195 & 1750.66 & 6.9989 & 671 & 21.3 \\
15 & 7400 & 1688.10 & 6.7488 & 620 & 19.6 \\
16 & 7428 & 1679.55 & 6.7146 & 532 & 16.8 \\
17 & 8696 & 1292.59 & 5.1676 & 303 & 9.6 \\
18 & 8705 & 1289.84 & 5.1566 & 330 & 10.5 \\
19 & 8725 & 1283.74 & 5.1322 & 375 & 11.9 \\
20 & 8734 & 1280.99 & 5.1212 & 361 & 11.4 \\
21 & 9543 & 1034.10 & 4.1342 & 219 & 6.9 \\
22 & 9552 & 1031.36 & 4.1232 & 242 & 7.7 \\
23 & 9575 & 1024.34 & 4.0952 & 463 & 14.7 \\
24 & 9584 & 1021.59 & 4.0842 & 440 & 13.9 \\
25 & 9636 & 1005.72 & 4.0208 & 434 & 13.7 \\
26 & 9666 & 996.57 & 3.9842 & 567 & 17.9 \\
27 & 9698 & 986.80 & 3.9451 & 258 & 8.2 \\
28 & 9830 & 946.52 & 3.7841 & 159 & 100.0 \\
\hline
\end{tabular}




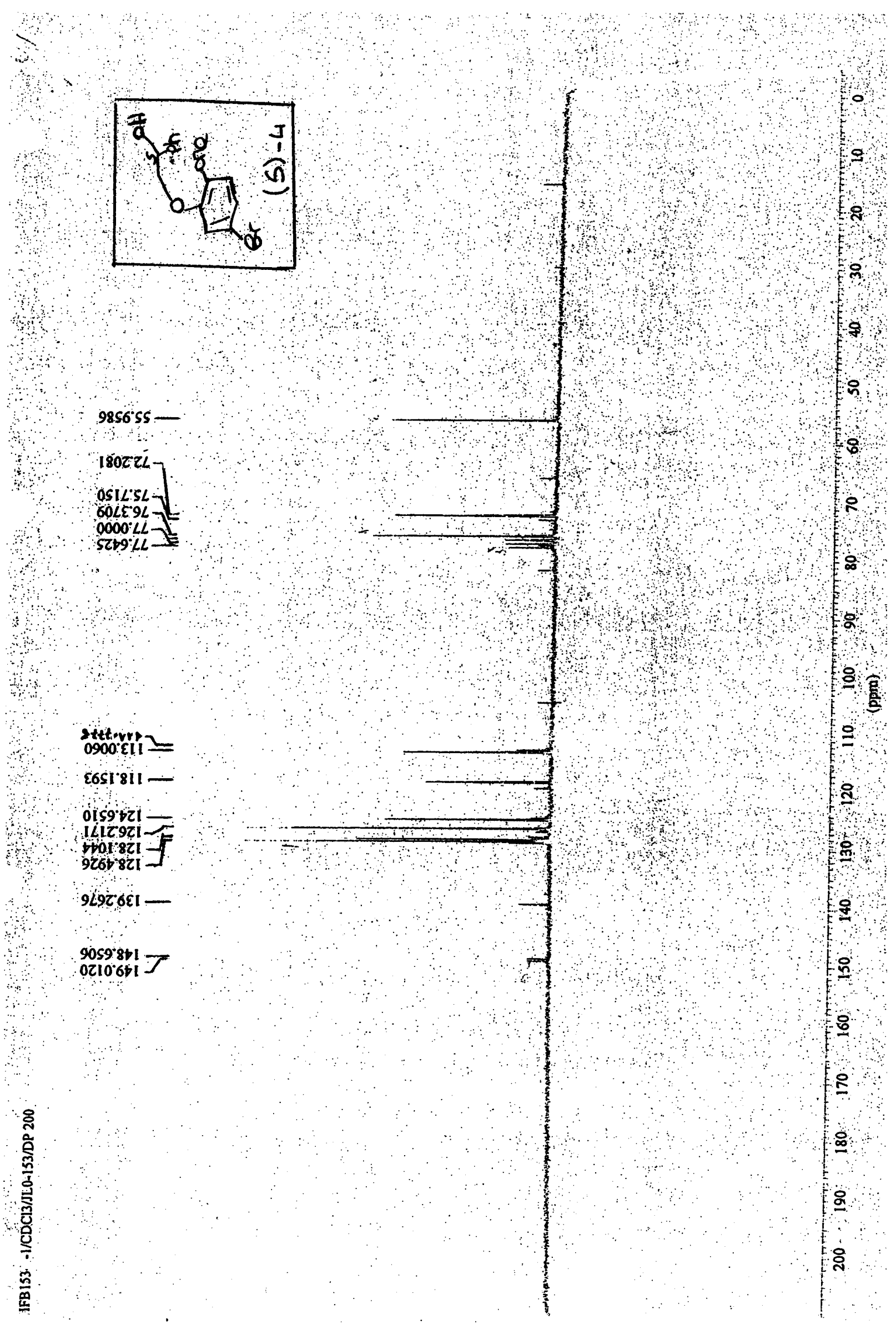




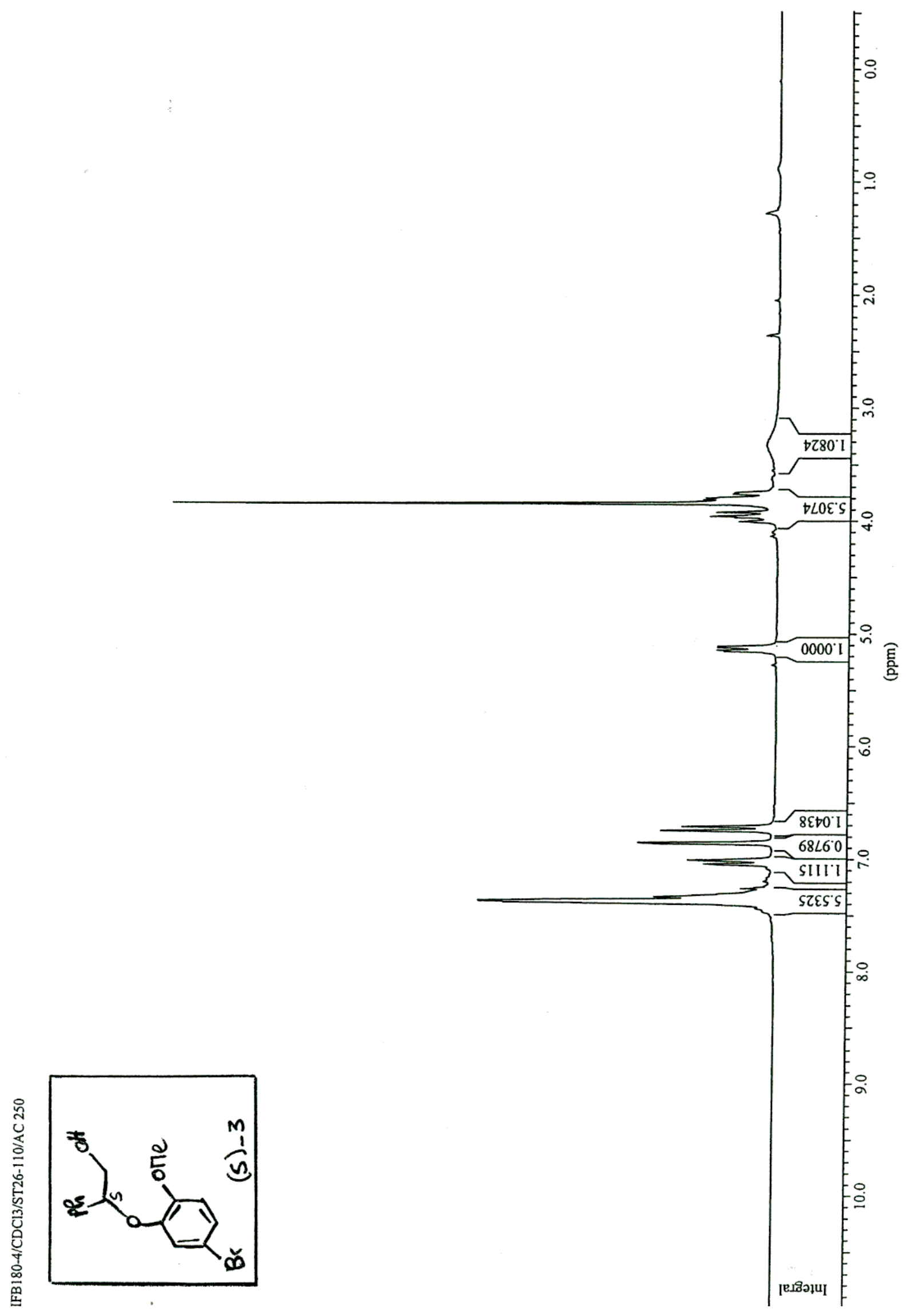


File Name

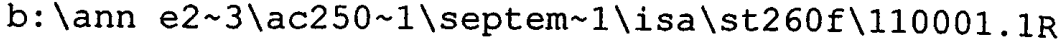
Peak Results saved in File : Nucleus $\mathrm{SF}$ OFFSET SW_p $S I$ $1 \mathrm{H}$ $250.132854 \mathrm{MHz}$ $15.7748 \mathrm{ppm}$ $5000.00 \mathrm{~Hz}$ : 16384

Peak Picking Parameter

Peak constant $\mathrm{PC}=$ Noise

1.00

1

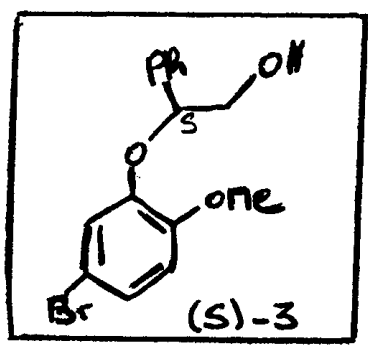

Sens. level

$=$

3

Peak Picking region

\begin{tabular}{rrrr} 
Start $(\mathrm{ppm} / \mathrm{Hz})$ & End $(\mathrm{ppm} / \mathrm{Hz})$ & MI $(8)$ & MAXI $\left(\frac{8}{8}\right)$ \\
\hline $5.28 / 1320.7$ & $4.89 / 1222.4$ & 7.62 & 18.68 \\
$4.04 / 1011.2$ & $3.43 / 857.7$ & 5.90 & 14.74 \\
$4.08 / 1019.8$ & $3.46 / 866.3$ & 91.91 & 108.37 \\
$3.39 / 847.0$ & $3.28 / 821.4$ & -0.00 & 6.39 \\
$6.88 / 1721.7$ & $6.78 / 1696.0$ & 20.89 & 26.29 \\
$7.11 / 1777.2$ & $6.93 / 1732.3$ & 10.32 & 18.43 \\
$6.78 / 1696.0$ & $6.65 / 1664.0$ & 12.78 & 22.61 \\
$7.54 / 1885.8$ & $7.21 / 1802.8$ & 17.45 & 53.57 \\
\hline
\end{tabular}

Peak Picking results

\begin{tabular}{rrrrrr} 
Peak Nr. Data Point & Frequency & \multicolumn{1}{c}{ PPM } & Intensity & zInt. \\
\hline 1 & 6885 & 1844.65 & 7.3747 & 1639 & 48.7 \\
2 & 6892 & 1842.51 & 7.3661 & 1643 & 48.8 \\
3 & 6920 & 1833.97 & 7.3320 & 664 & 19.7 \\
4 & 7158 & 1761.34 & 7.0416 & 389 & 11.6 \\
5 & 7187 & 1752.49 & 7.0062 & 480 & 14.3 \\
6 & 7313 & 1714.04 & 6.8525 & 753 & 22.4 \\
7 & 7401 & 1687.18 & 6.7451 & 630 & 18.7 \\
8 & 7430 & 1678.33 & 6.7098 & 511 & 15.2 \\
9 & 8706 & 1288.93 & 5.1530 & 290 & 8.6 \\
10 & 8715 & 1286.18 & 5.1420 & 331 & 9.8 \\
11 & 8733 & 1280.69 & 5.1200 & 327 & 9.7 \\
12 & 8743 & 1277.63 & 5.1078 & 326 & 9.7 \\
13 & 9651 & 1000.53 & 4.0000 & 218 & 6.5 \\
14 & 9679 & 991.99 & 3.9659 & 242 & 7.2 \\
15 & 9690 & 988.63 & 3.9524 & 376 & 11.2 \\
16 & 9718 & 980.09 & 3.9183 & 340 & 10.1 \\
17 & 9781 & 960.86 & 3.8414 & 3363 & 100.0 \\
18 & 9799 & 955.37 & 3.8194 & 392 & 11.7 \\
19 & 9810 & 952.01 & 3.8060 & 415 & 12.3 \\
20 & 9821 & 948.65 & 3.7926 & 402 & 11.9 \\
21 & 9850 & 939.80 & 3.7572 & 252 & 7.5 \\
22 & 9860 & 936.75 & 3.7450 & 242 & 7.2 \\
23 & 10203 & 832.08 & 3.3265 & 69 & 2.0 \\
\hline
\end{tabular}


9806 ऽs -

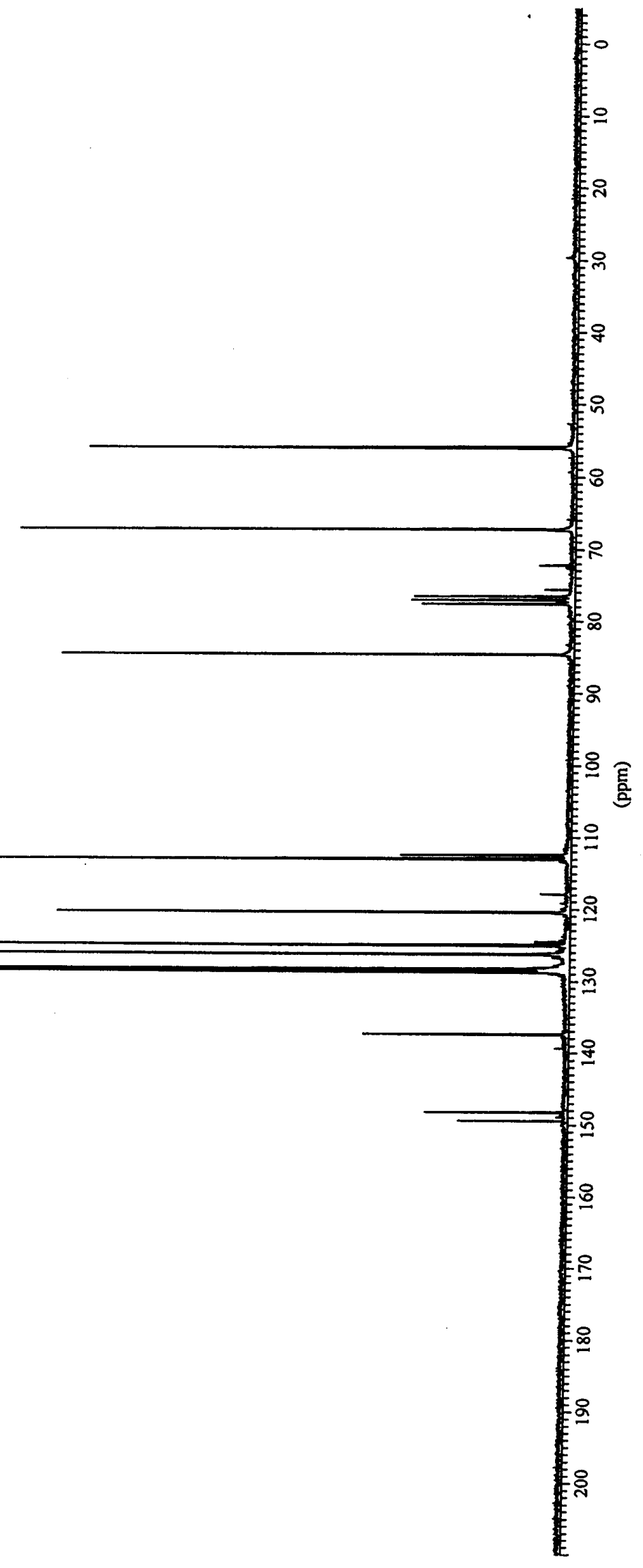

0S0t'ZII

${ }_{8056} 211$ L

908E0Z1 -

$\angle 656 \circ 21$

6L81'921-

zt+9.821]

$9 \angle \downarrow \varepsilon \angle \varepsilon I-$

8ELI $8 t+$

$0200^{6} 601-$

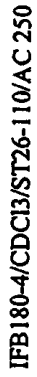

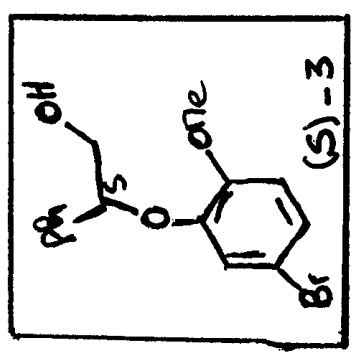

$\infty$ 


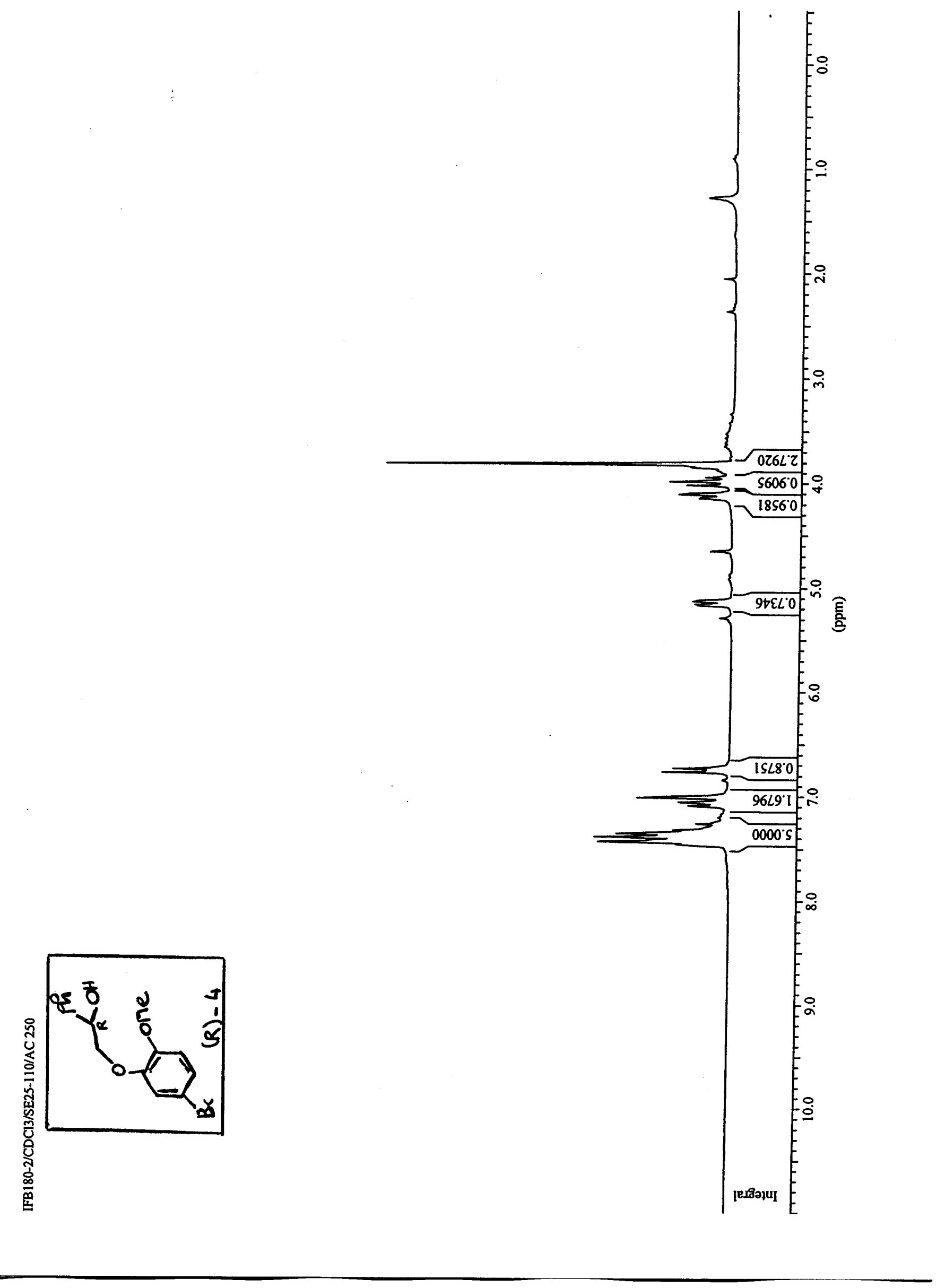


File Name : C $:$ WIN1D \SPC \ASP3000\SE25\110001.1R

Peak Results saved in File:

Nucleus

$\mathrm{SF}$

OFFSET

SW_p

SI

$1 \mathrm{H}$

$250.132854 \mathrm{MHz}$

$15.7760 \mathrm{ppm}$

$5000.00 \mathrm{~Hz}$

16384

Peak Picking Parameter

$\begin{array}{lrr}\text { Peak constant PC } & = & 1.00 \\ \text { Noise } & = & 1 \\ \text { Sens. level } & = & 5\end{array}$

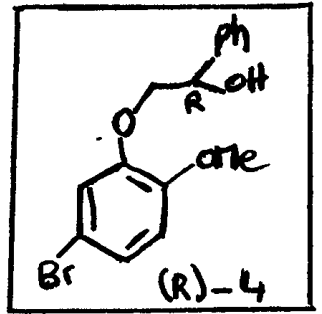

Peak Picking region

\begin{tabular}{rrrr} 
Start $(\mathrm{ppm} / \mathrm{Hz})$ & End $(\mathrm{ppm} / \mathrm{Hz})$ & MI $(q)$ & MAXI $(\%)$ \\
\hline $5.25 / 1314.3$ & $5.02 / 1255.4$ & 8.80 & 11.86 \\
$4.17 / 1042.3$ & $4.07 / 1017.9$ & 7.83 & 19.39 \\
$4.03 / 1008.5$ & $3.90 / 974.6$ & 6.63 & 19.09 \\
$3.83 / 957.2$ & $3.69 / 923.9$ & 94.51 & 106.72 \\
$6.78 / 1695.1$ & $6.62 / 1655.7$ & 10.28 & 23.68 \\
$7.02 / 1755.2$ & $6.92 / 1731.1$ & 19.08 & 32.49 \\
$7.14 / 1786.4$ & $7.04 / 1761.9$ & 10.28 & 22.48 \\
$7.47 / 1868.5$ & $7.25 / 1812.6$ & 7.88 & 49.69 \\
\hline
\end{tabular}

\section{Peak Picking results}

\begin{tabular}{rrrrrr} 
Peak Nr. Data Point & Frequency & \multicolumn{1}{c}{ PPM } & Intensity & o Int. \\
\hline 1 & 6817 & 1865.71 & 7.4589 & 509 & 15.3 \\
2 & 6840 & 1858.69 & 7.4308 & 1260 & 37.9 \\
3 & 6878 & 1847.09 & 7.3844 & 1293 & 38.9 \\
4 & 6903 & 1839.46 & 7.3539 & 1077 & 32.4 \\
5 & 6929 & 1831.53 & 7.3222 & 538 & 16.2 \\
6 & 6980 & 1815.96 & 7.2600 & 314 & 9.5 \\
7 & 7123 & 1772.32 & 7.0855 & 393 & 11.8 \\
8 & 7151 & 1763.78 & 7.0514 & 490 & 14.7 \\
9 & 7185 & 1753.40 & 7.0099 & 887 & 26.7 \\
10 & 7388 & 1691.45 & 6.7622 & 642 & 19.3 \\
11 & 7416 & 1682.91 & 6.7281 & 540 & 16.3 \\
12 & 8698 & 1291.67 & 5.1639 & 335 & 10.1 \\
13 & 8706 & 1289.23 & 5.1542 & 357 & 10.7 \\
14 & 8727 & 1282.82 & 5.1286 & 376 & 11.3 \\
15 & 8736 & 1280.08 & 5.1176 & 352 & 10.6 \\
16 & 9535 & 1036.24 & 4.1428 & 330 & 9.9 \\
17 & 9544 & 1033.49 & 4.1318 & 314 & 9.5 \\
18 & 9567 & 1026.47 & 4.1037 & 523 & 15.7 \\
19 & 9575 & 1024.03 & 4.0940 & 482 & 14.5 \\
20 & 9639 & 1004.50 & 4.0159 & 446 & 13.4 \\
21 & 9670 & 995.04 & 3.9781 & 607 & 18.3 \\
22 & 9701 & 985.58 & 3.9402 & 270 & 8.1 \\
23 & 9810 & 952.32 & 3.8072 & 3321 & 100.0 \\
\hline
\end{tabular}




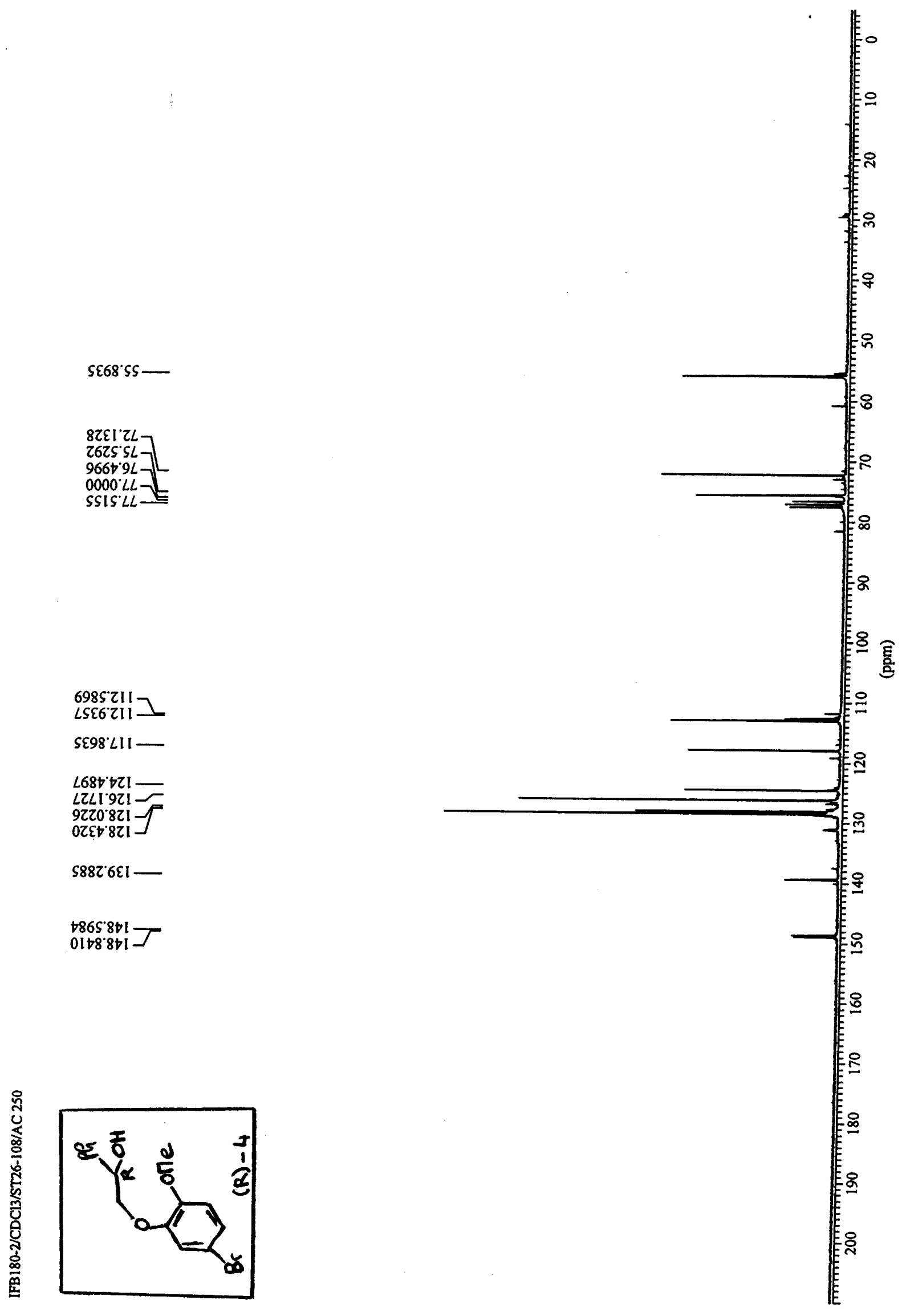



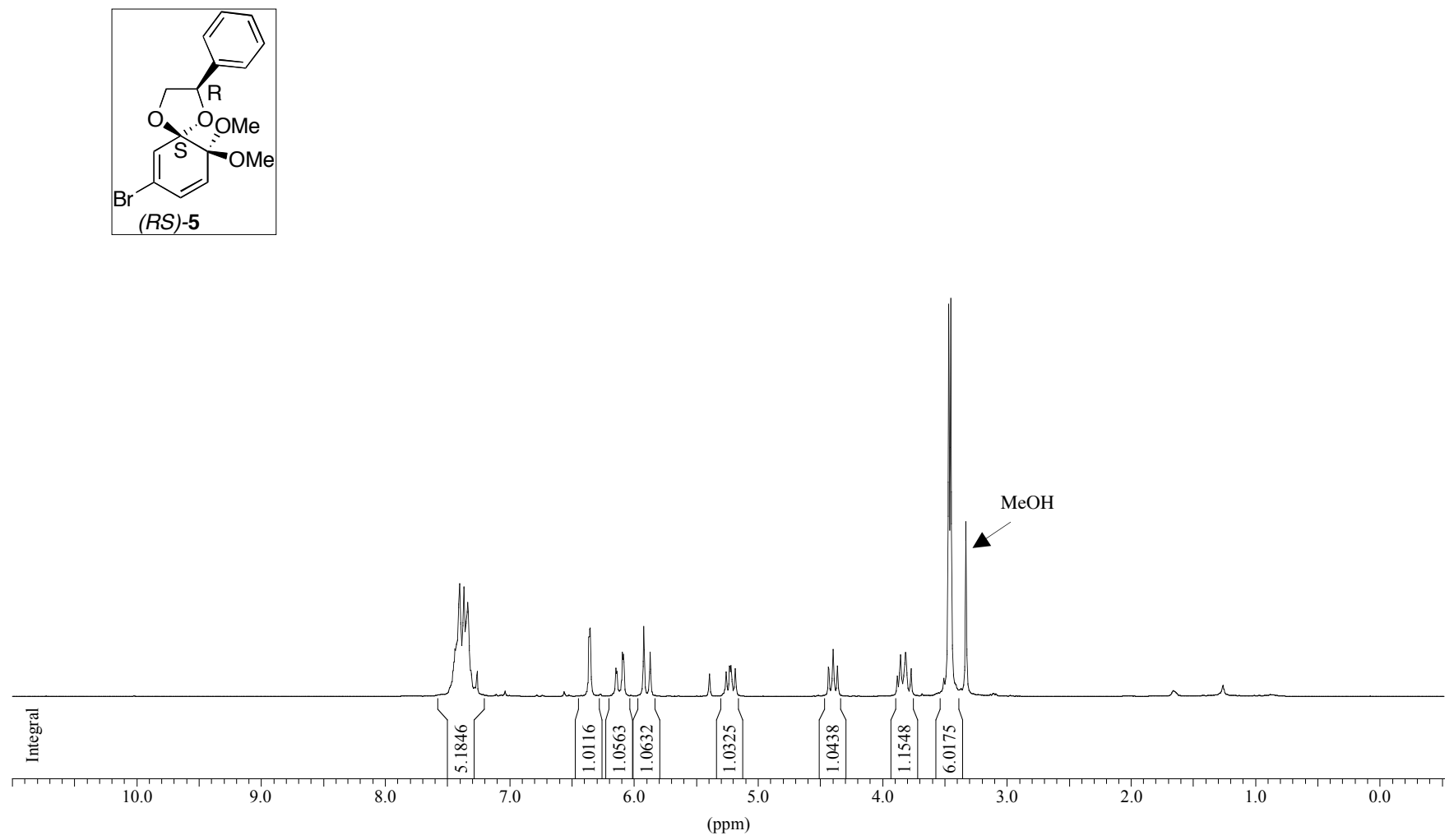

\begin{tabular}{rrrrrr}
\hline & & & & & \\
Peak Nr. & Data Point & Frequency & PPM & Intensity & \%Int. \\
\hline 1 & 5632 & 1488.61 & 7.4371 & 19608 & 12.0 \\
2 & 5677 & 1481.45 & 7.4013 & 46272 & 28.4 \\
3 & 5719 & 1474.78 & 7.3680 & 44857 & 27.5 \\
4 & 5760 & 1468.26 & 7.3354 & 38537 & 23.6 \\
5 & 5855 & 1453.16 & 7.2600 & 10062 & 6.2 \\
6 & 6988 & 1273.08 & 6.3603 & 26895 & 16.5 \\
7 & 6997 & 1271.65 & 6.3531 & 28084 & 17.2 \\
8 & 7260 & 1229.84 & 6.1443 & 11507 & 7.1 \\
9 & 7270 & 1228.25 & 6.1364 & 10830 & 6.6 \\
10 & 7325 & 1219.51 & 6.0927 & 18176 & 11.1 \\
11 & 7335 & 1217.92 & 6.0847 & 17265 & 10.6 \\
12 & 7542 & 1185.02 & 5.9204 & 28740 & 17.6 \\
13 & 7607 & 1174.69 & 5.8687 & 18264 & 11.2 \\
14 & 8372 & 1053.10 & 5.2613 & 9896 & 6.1 \\
15 & 8414 & 1046.42 & 5.2279 & 12419 & 7.6 \\
16 & 8429 & 1044.04 & 5.2160 & 12639 & 7.7 \\
17 & 8471 & 1037.36 & 5.1827 & 11200 & 6.9 \\
18 & 9415 & 887.32 & 4.4330 & 11850 & 7.3 \\
19 & 9461 & 880.00 & 4.3965 & 19410 & 11.9 \\
20 & 9505 & 873.01 & 4.3616 & 12366 & 7.6 \\
21 & 10110 & 776.85 & 3.8811 & 8223 & 5.0 \\
22 & 10144 & 771.44 & 3.8541 & 16978 & 10.4 \\
23 & 10193 & 763.66 & 3.8152 & 18010 & 11.0 \\
24 & 10633 & 693.72 & 3.4658 & 160796 & 98.6 \\
25 & 10653 & 690.54 & 3.4499 & 163117 & 100.0 \\
\hline & & & & &
\end{tabular}

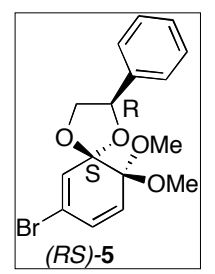


8020'Is

06SI'IS

$52 \angle 0^{\circ} 1 L$

0000 LL

$8126 \angle L$

$00028 L$

$8000^{\circ} \mathrm{L6}$

$\angle 90 S^{\circ} 80 \mathrm{I}$

Eb66 8II -

0I6ع.9ZI -

8E9t'8ZI 7

S919.8ZI - E

I28t. IEI

†68Z ZEI

98E9.9EI

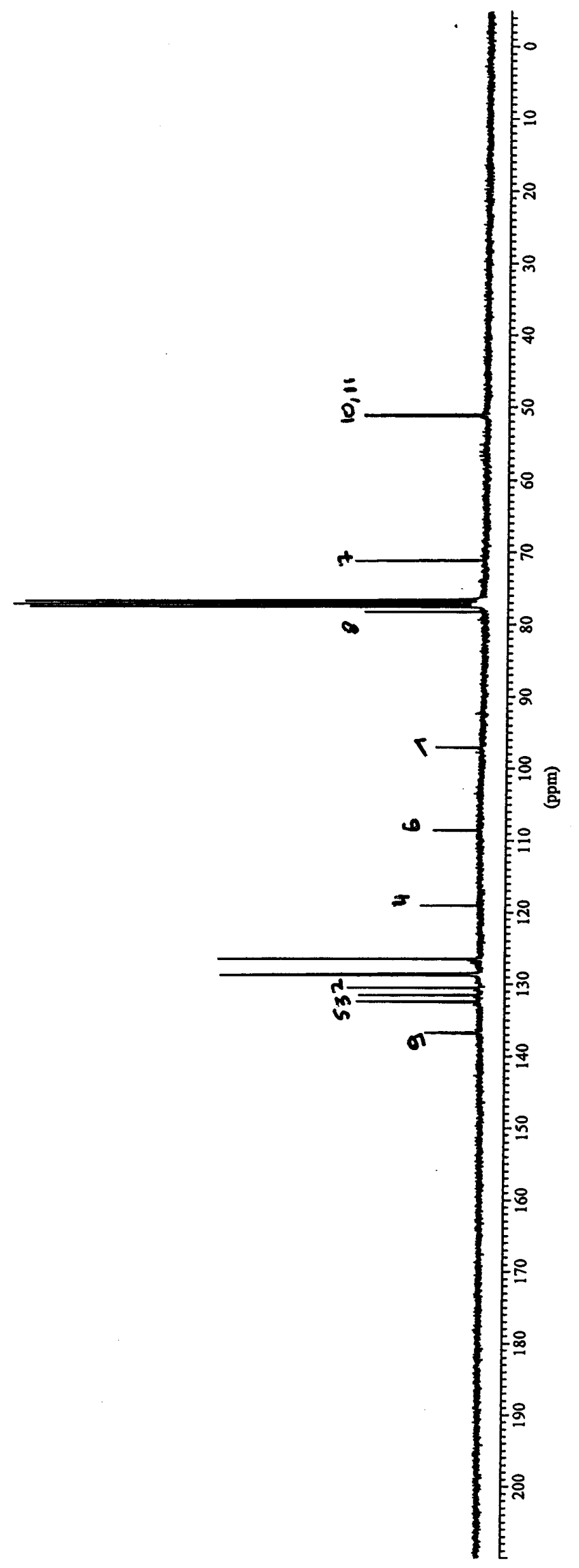




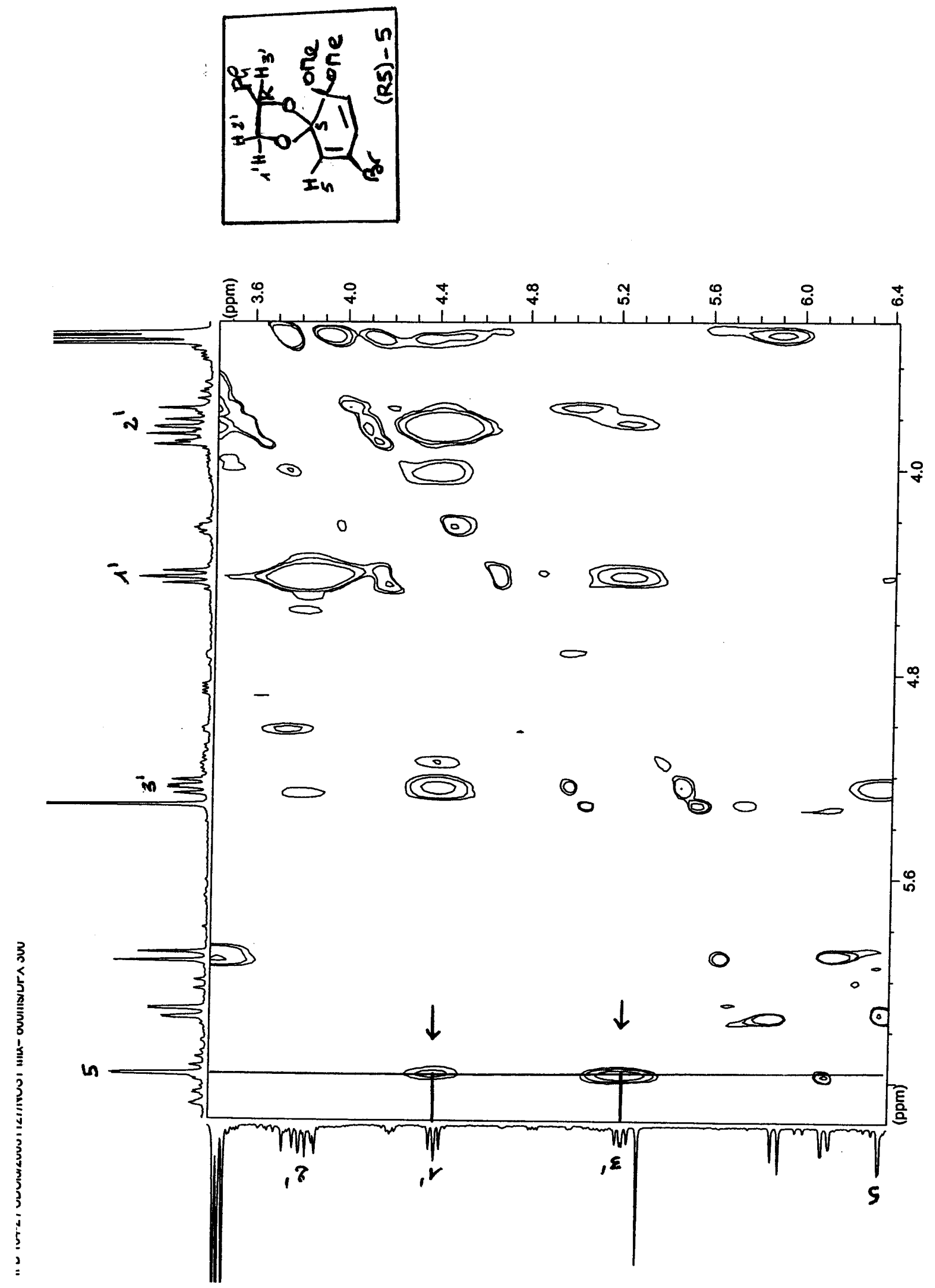



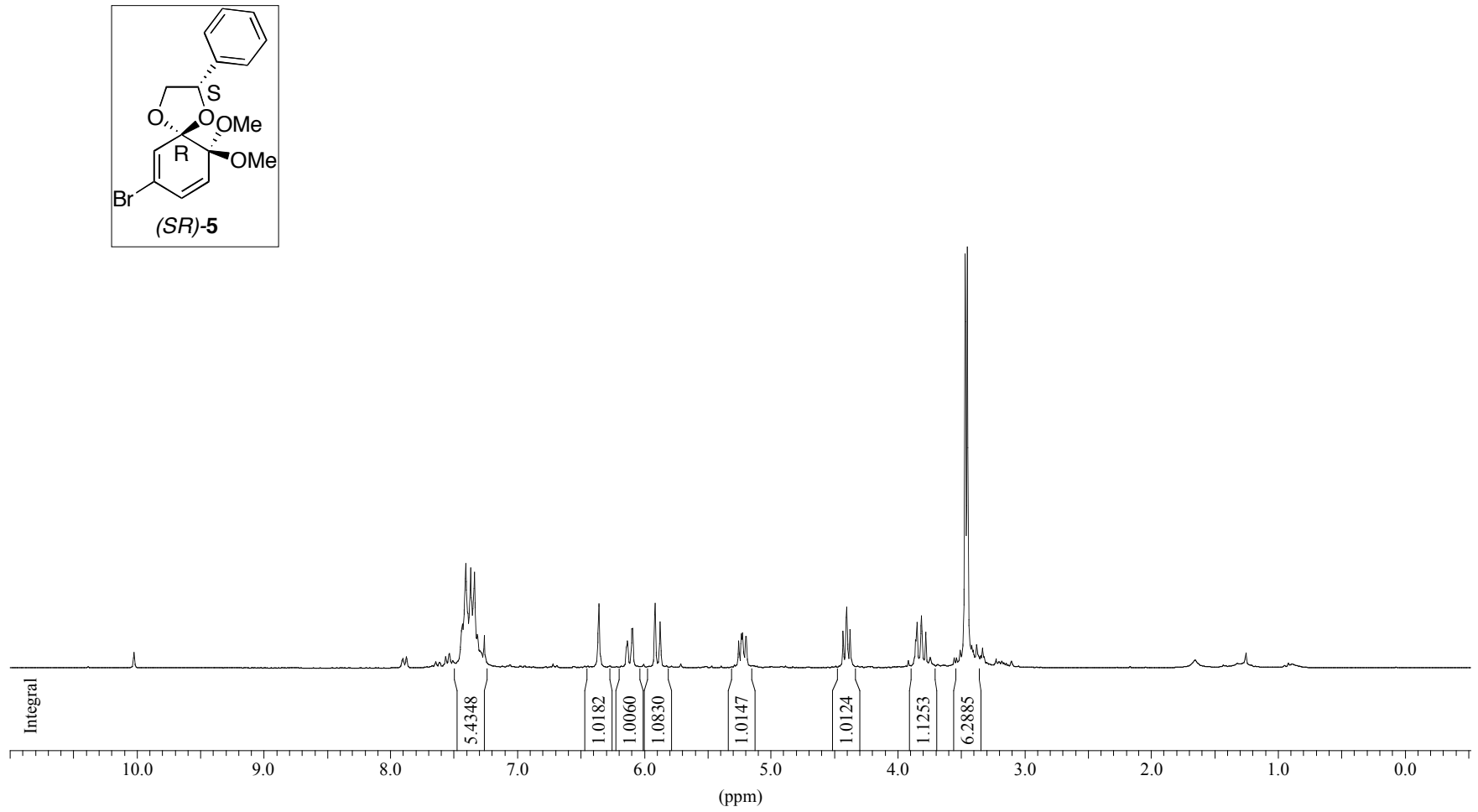

\begin{tabular}{crrrrr}
\hline Peak Nr. & Data Point & Frequency & PPM & Intensity & \%lnt. \\
\hline 1 & 6837 & 1859.30 & 7.4332 & 498 & 10.3 \\
2 & 6861 & 1851.98 & 7.4040 & 1200 & 24.8 \\
3 & 6891 & 1842.82 & 7.3674 & 1153 & 23.8 \\
4 & 6916 & 1835.19 & 7.3369 & 1097 & 22.7 \\
5 & 6935 & 1829.39 & 7.3137 & 380 & 7.8 \\
6 & 6979 & 1815.96 & 7.2600 & 368 & 7.6 \\
7 & 7716 & 1591.05 & 6.3608 & 682 & 14.1 \\
8 & 7721 & 1589.52 & 6.3547 & 735 & 15.2 \\
9 & 7898 & 1535.51 & 6.1388 & 301 & 6.2 \\
10 & 7903 & 1533.98 & 6.1327 & 304 & 6.3 \\
11 & 7932 & 1525.13 & 6.0973 & 439 & 9.1 \\
12 & 7937 & 1523.61 & 6.0912 & 446 & 9.2 \\
13 & 8082 & 1479.36 & 5.9143 & 742 & 15.3 \\
14 & 8115 & 1469.28 & 5.8740 & 527 & 10.9 \\
15 & 8621 & 1314.87 & 5.2567 & 304 & 6.3 \\
16 & 8643 & 1308.15 & 5.2298 & 385 & 7.9 \\
17 & 8651 & 1305.71 & 5.2201 & 395 & 8.2 \\
18 & 8673 & 1299.00 & 5.1932 & 354 & 7.3 \\
19 & 9299 & 1107.96 & 4.4295 & 416 & 8.6 \\
20 & 9323 & 1100.63 & 4.4002 & 697 & 14.4 \\
21 & 9345 & 1093.92 & 4.3734 & 435 & 9.0 \\
22 & 9777 & 962.08 & 3.8463 & 514 & 10.6 \\
23 & 9806 & 953.23 & 3.8109 & 593 & 12.2 \\
24 & 9832 & 945.30 & 3.7792 & 403 & 8.3 \\
25 & 10087 & 867.48 & 3.4681 & 4763 & 98.3 \\
26 & 10101 & 863.21 & 3.4510 & 4843 & 100.0 \\
\hline & & & & &
\end{tabular}

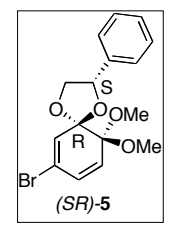


I026.0S

LILO'IS

ZS96.0L

St8 $80^{\circ} 9 L$

$0000^{\circ} \mathrm{LL}$

$000 S^{\circ} L L$

$\angle I 600^{\circ} \mathrm{L}$

$\varsigma \varepsilon 68 \% 96$

$898 \varepsilon 80 \mathrm{I}-$

9768.811

0t67'92I -

$\varepsilon \mathrm{IL \varepsilon} 8 \mathrm{8Z}$ I

6ZZS $821-$

OL6Z0EI

06It IEI

SLOZ'ZદI
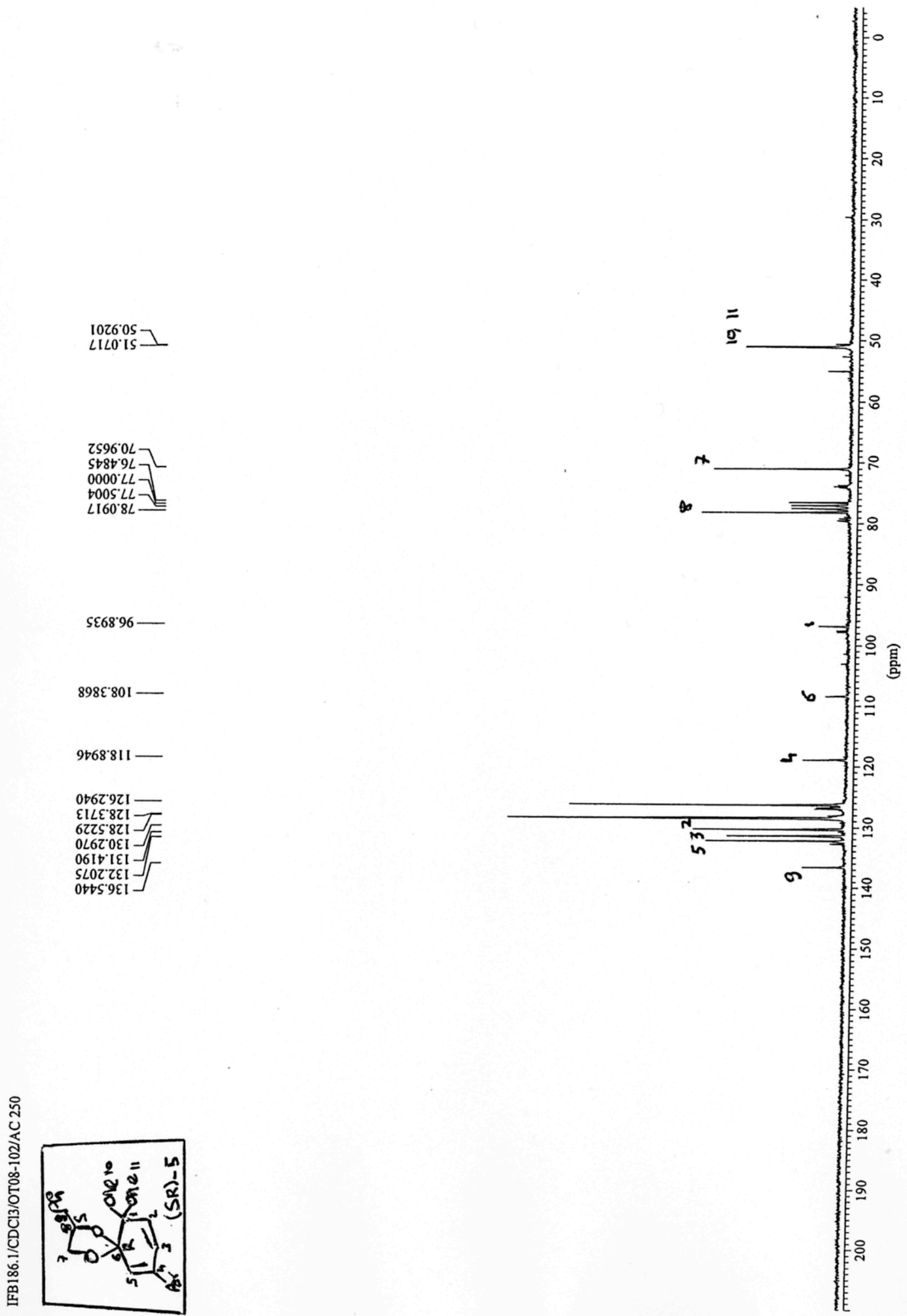

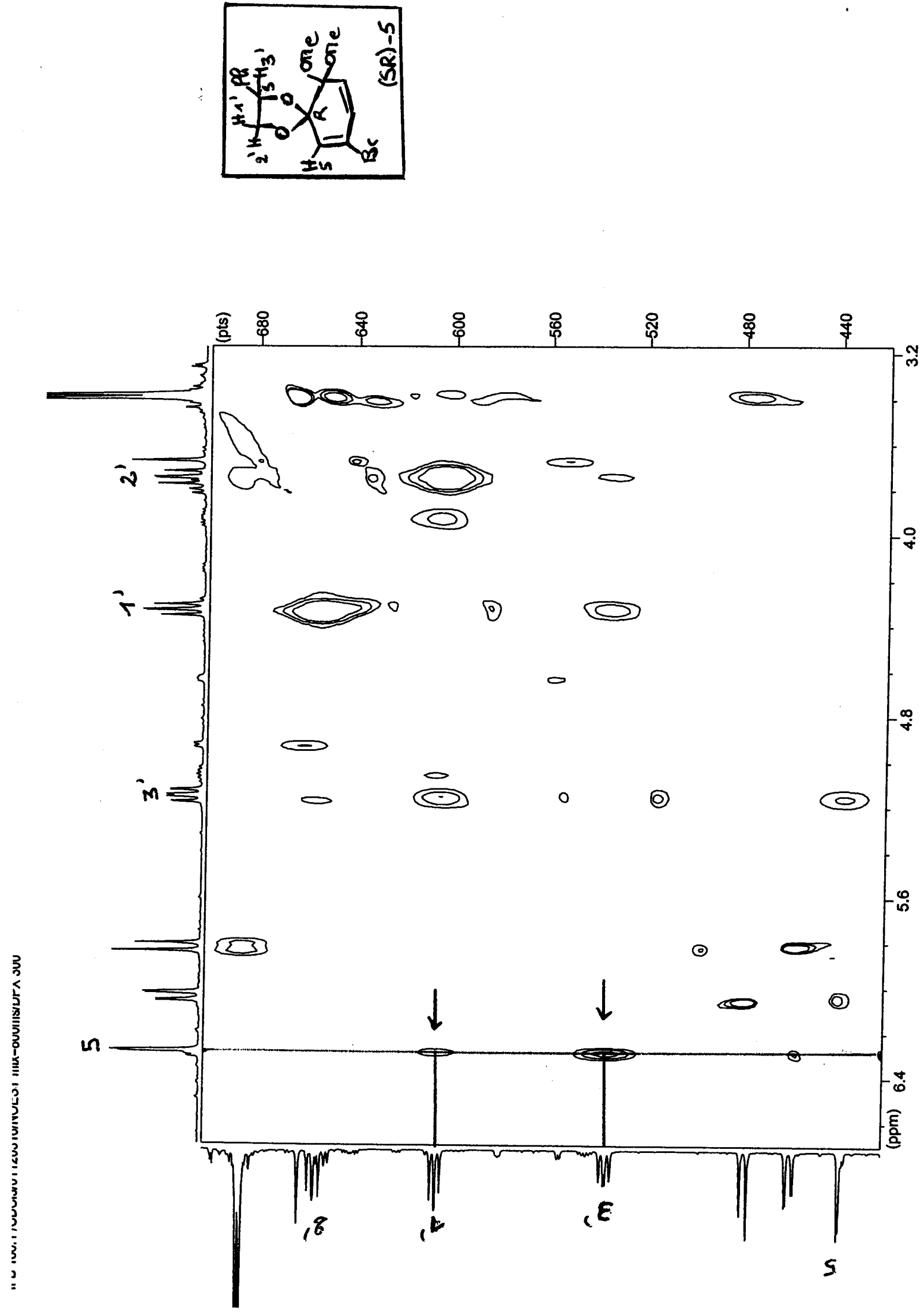

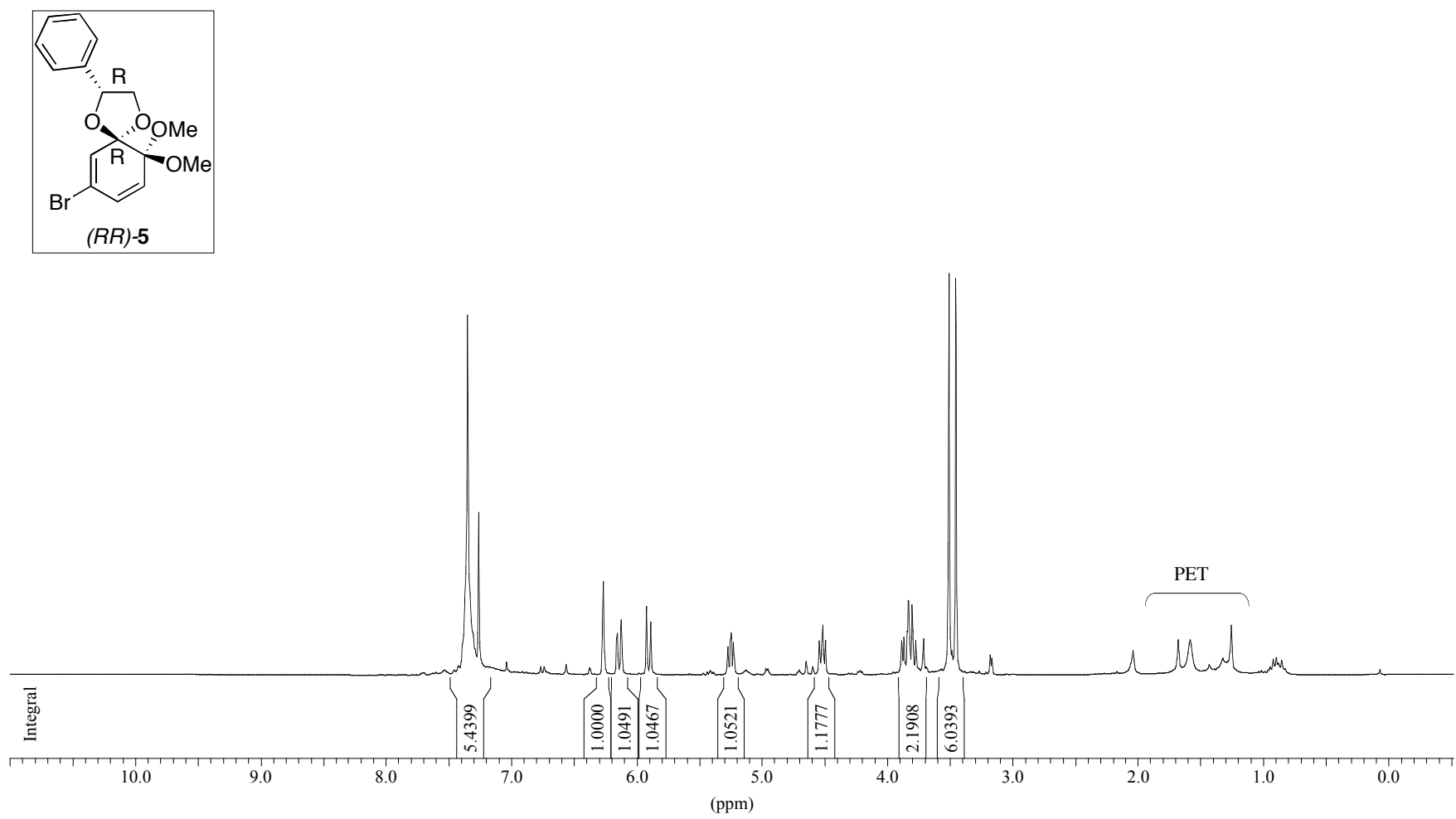

\begin{tabular}{crrrrr}
\hline & & & & & \\
Peak Nr. & Data Point & Frequency & PPM & Intensity & \%lnt. \\
\hline 1 & 9889 & 2205.69 & 7.3491 & 260032 & 89.7 \\
2 & 9960 & 2178.94 & 7.2600 & 117446 & 40.5 \\
3 & 10752 & 1880.55 & 6.2658 & 67472 & 23.3 \\
4 & 10839 & 1847.77 & 6.1566 & 29818 & 10.3 \\
5 & 10866 & 1837.60 & 6.1227 & 39751 & 13.7 \\
6 & 11025 & 1777.69 & 5.9231 & 49682 & 17.1 \\
7 & 11052 & 1767.52 & 5.8892 & 38401 & 13.2 \\
8 & 11541 & 1583.29 & 5.2753 & 20181 & 7.0 \\
9 & 11563 & 1575.00 & 5.2477 & 30213 & 10.4 \\
10 & 11581 & 1568.22 & 5.2251 & 23057 & 8.0 \\
11 & 12125 & 1363.26 & 4.5422 & 24373 & 8.4 \\
12 & 12142 & 1356.85 & 4.5209 & 29225 & 10.1 \\
13 & 12147 & 1354.97 & 4.5146 & 35659 & 12.3 \\
14 & 12164 & 1348.56 & 4.4933 & 24822 & 8.6 \\
15 & 12647 & 1166.59 & 3.8869 & 24435 & 8.4 \\
16 & 12662 & 1160.94 & 3.8681 & 27123 & 9.4 \\
17 & 12691 & 1150.01 & 3.8317 & 53867 & 18.6 \\
18 & 12715 & 1140.97 & 3.8016 & 50788 & 17.5 \\
19 & 12737 & 1132.68 & 3.7740 & 24605 & 8.5 \\
20 & 12787 & 1113.84 & 3.7112 & 25671 & 8.9 \\
21 & 12948 & 1053.18 & 3.5091 & 289976 & 100.0 \\
22 & 12992 & 1036.61 & 3.4539 & 286469 & 98.8 \\
\hline
\end{tabular}

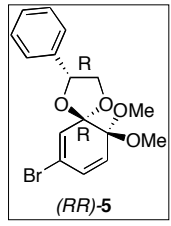




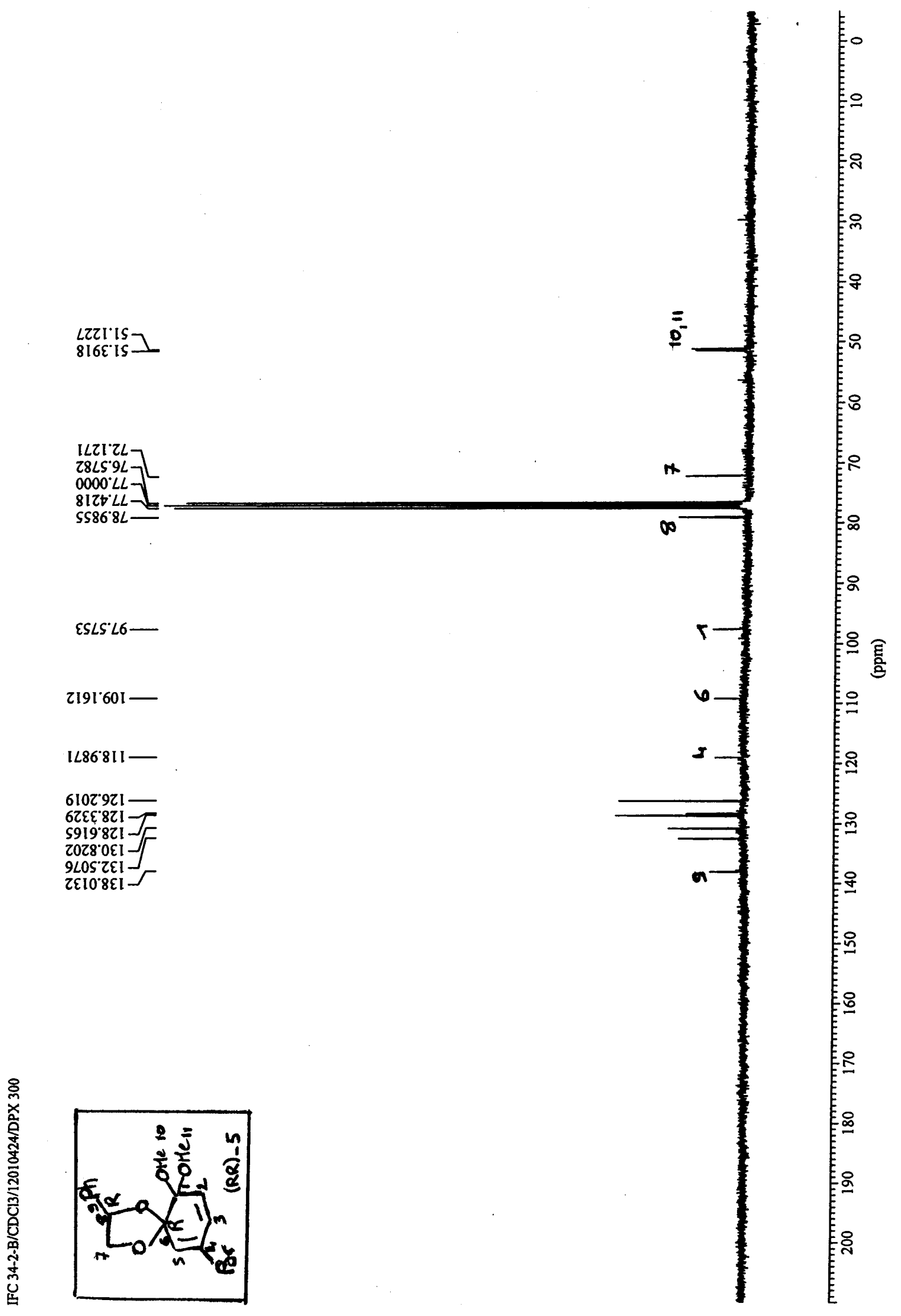



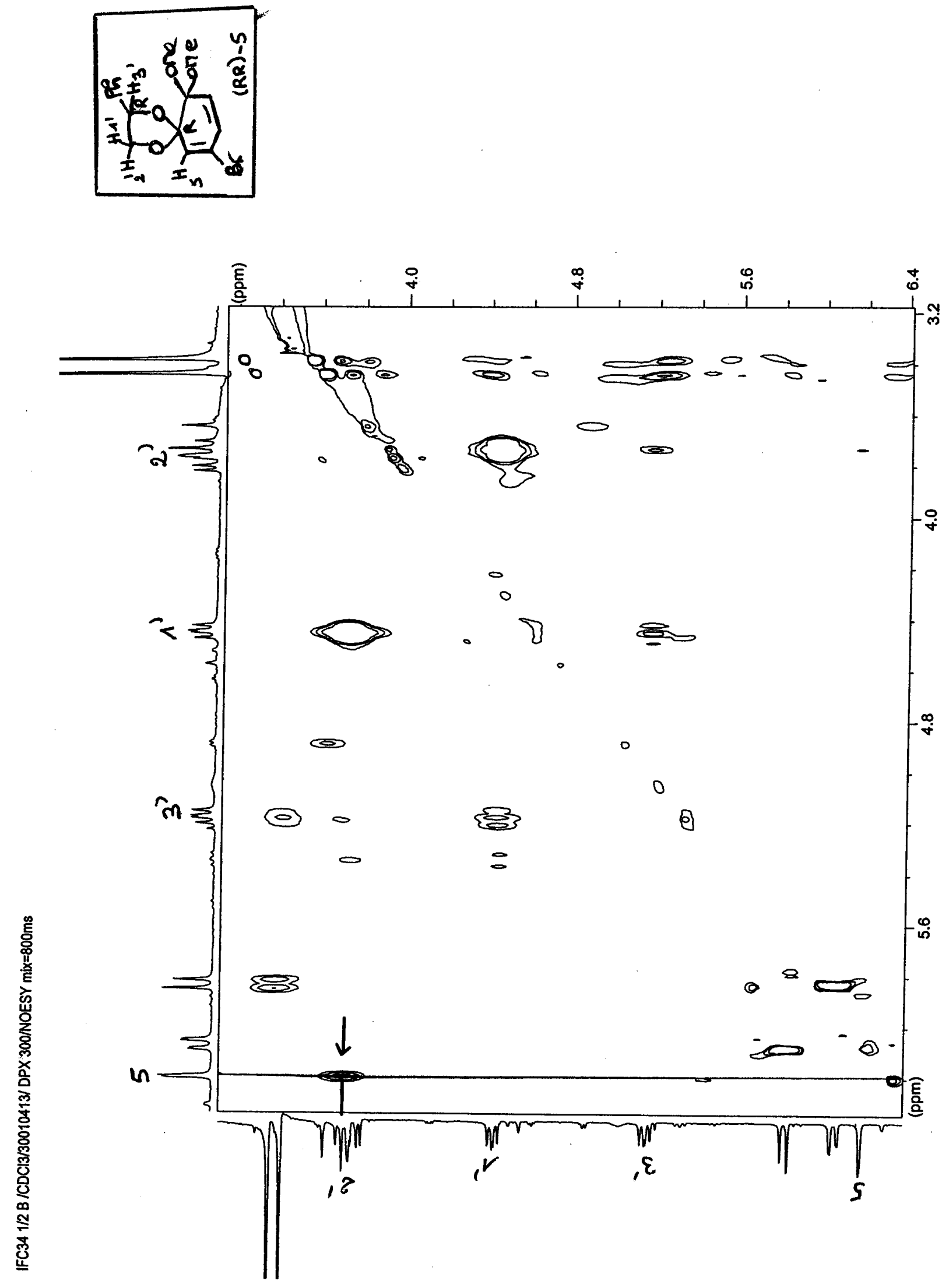

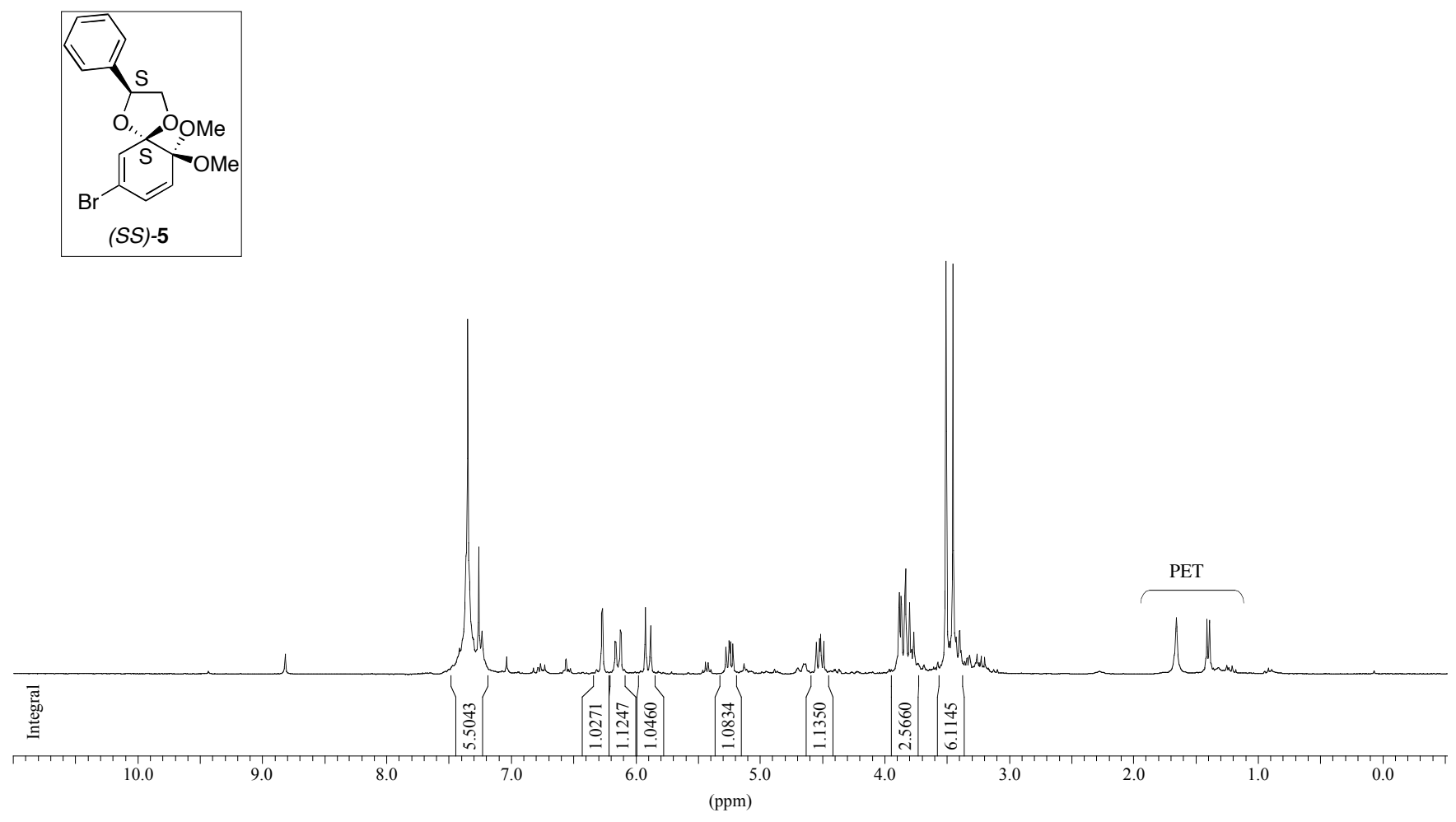

\begin{tabular}{|c|c|c|c|c|c|}
\hline Peak Nr. & Data Point & Frequency & PPM & Intensity & \%int. \\
\hline 1 & 6907 & 1837.94 & 7.3478 & 3103 & 85.8 \\
\hline 2 & 6917 & 1834.89 & 7.3356 & 959 & 26.5 \\
\hline 3 & 6979 & 1815.96 & 7.2600 & 1109 & 30.7 \\
\hline 4 & 7788 & 1569.08 & 6.2730 & 532 & 14.7 \\
\hline 5 & 7794 & 1567.25 & 6.2657 & 572 & 15.8 \\
\hline 6 & 7876 & 1542.22 & 6.1656 & 285 & 7.9 \\
\hline 7 & 7881 & 1540.70 & 6.1595 & 274 & 7.6 \\
\hline 8 & 7909 & 1532.15 & 6.1253 & 385 & 10.7 \\
\hline 9 & 7915 & 1530.32 & 6.1180 & 355 & 9.8 \\
\hline 10 & 8075 & 1481.49 & 5.9228 & 587 & 16.2 \\
\hline 11 & 8109 & 1471.12 & 5.8813 & 420 & 11.6 \\
\hline 12 & 8605 & 1319.75 & 5.2762 & 234 & 6.5 \\
\hline 13 & 8626 & 1313.34 & 5.2506 & 291 & 8.1 \\
\hline 14 & 8634 & 1310.90 & 5.2408 & 279 & 7.7 \\
\hline 15 & 8654 & 1304.80 & 5.2164 & 261 & 7.2 \\
\hline 16 & 9202 & 1137.56 & 4.5478 & 279 & 7.7 \\
\hline 17 & 9223 & 1131.15 & 4.5222 & 311 & 8.6 \\
\hline 18 & 9230 & 1129.01 & 4.5137 & 347 & 9.6 \\
\hline 19 & 9251 & 1122.61 & 4.4880 & 284 & 7.8 \\
\hline 20 & 9748 & 970.93 & 3.8817 & 718 & 19.9 \\
\hline 21 & 9760 & 967.27 & 3.8670 & 685 & 18.9 \\
\hline 22 & 9786 & 959.34 & 3.8353 & 880 & 24.3 \\
\hline 23 & 9789 & 958.42 & 3.8316 & 920 & 25.5 \\
\hline 24 & 9817 & 949.88 & 3.7975 & 629 & 17.4 \\
\hline 25 & 9845 & 941.33 & 3.7633 & 365 & 10.1 \\
\hline 26 & 10055 & 877.24 & 3.5071 & 3615 & 100.0 \\
\hline 27 & 10101 & 863.21 & 3.4510 & 3590 & 99.3 \\
\hline
\end{tabular}

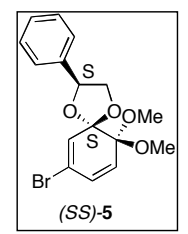


$\underset{S+8 E \cdot I S>}{t S I I I S}$

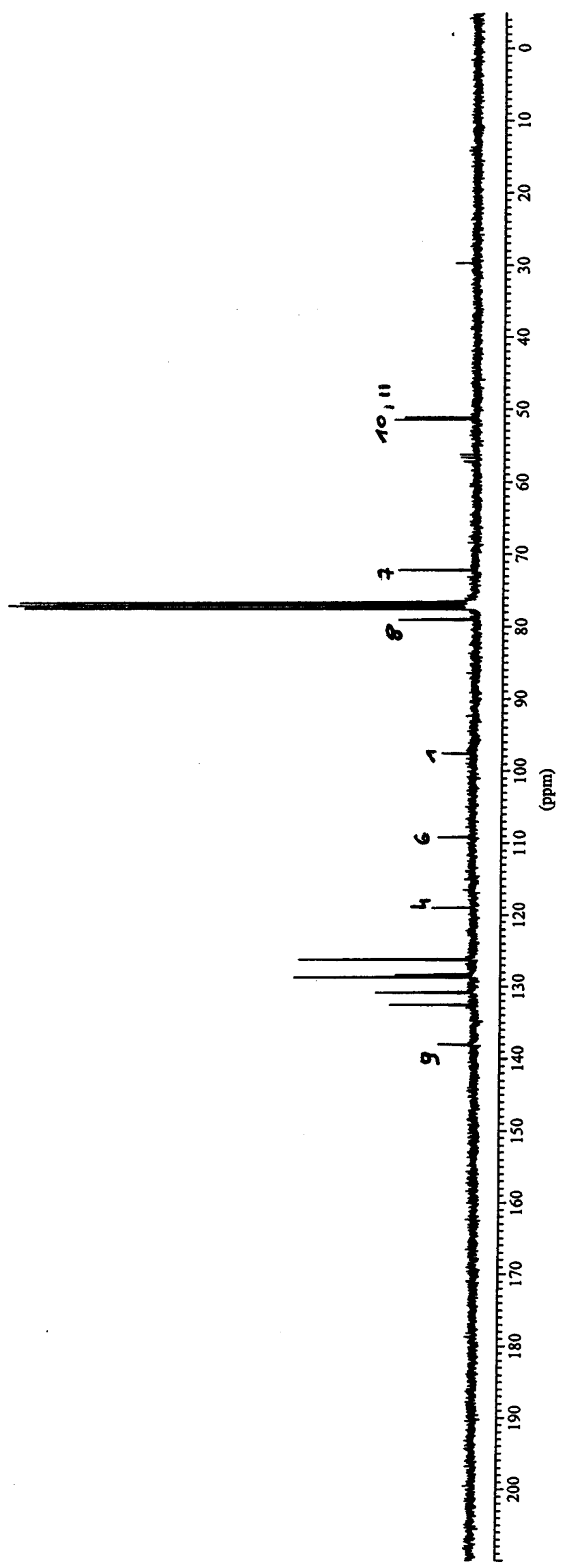



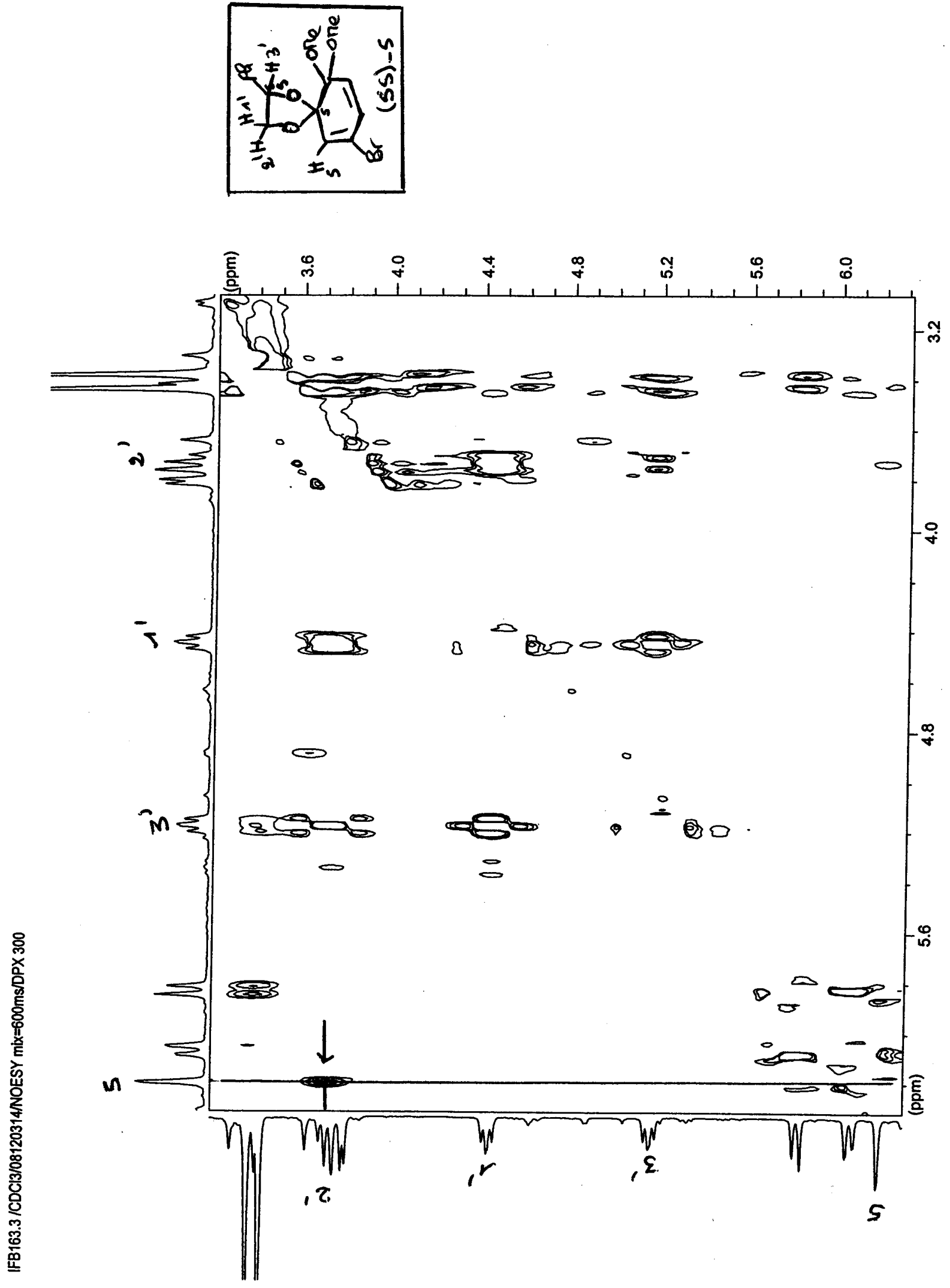

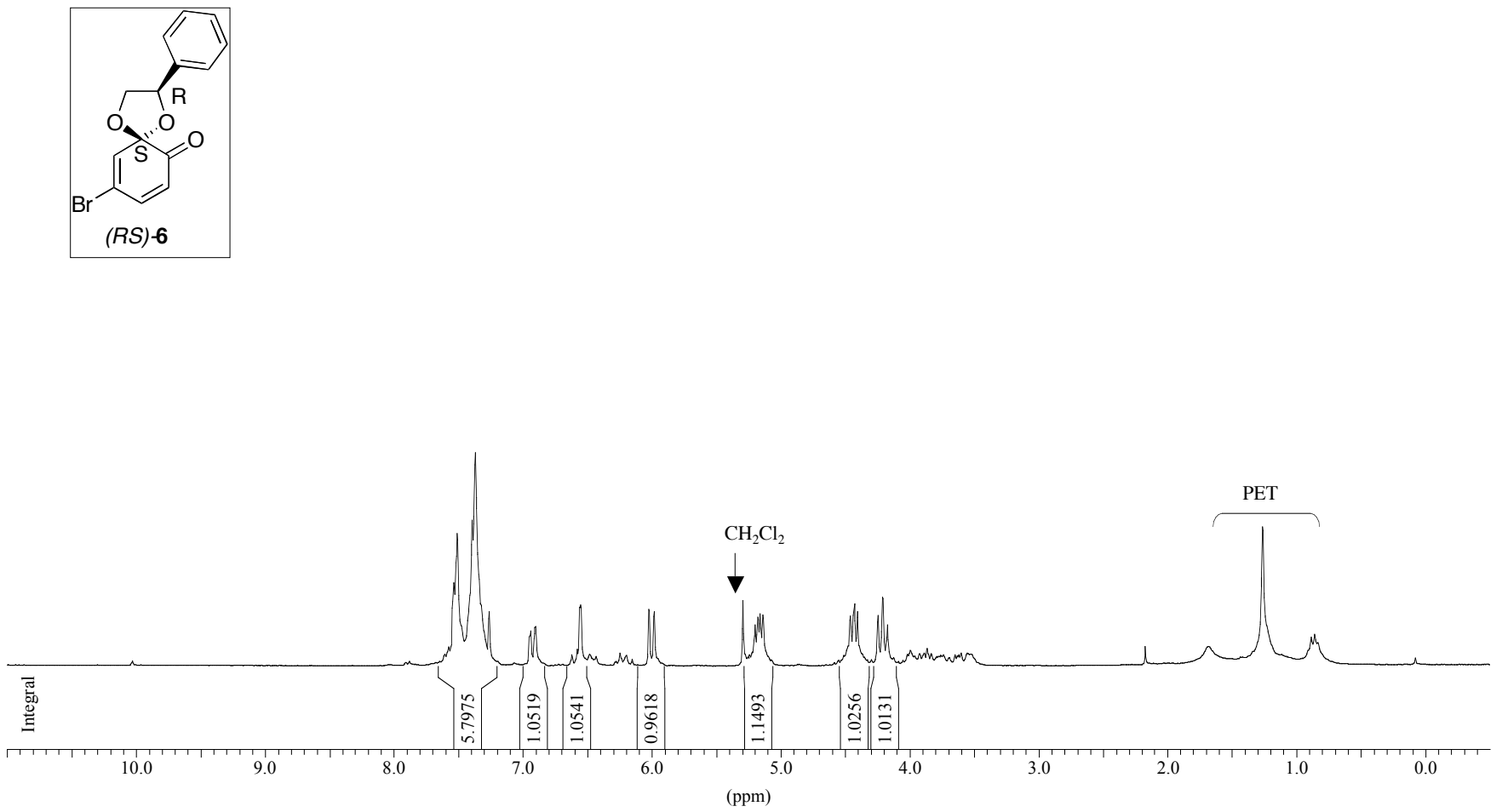

\begin{tabular}{rrrrrr}
\hline Peak Nr. & Data Point & Frequency & PPM & Intensity & \%lnt. \\
\hline 1 & 6754 & 1884.93 & 7.5357 & 380 & 39.3 \\
2 & 6776 & 1878.22 & 7.5089 & 601 & 62.2 \\
3 & 6870 & 1849.53 & 7.3942 & 661 & 68.4 \\
4 & 6892 & 1842.82 & 7.3674 & 966 & 100.0 \\
5 & 6980 & 1815.96 & 7.2600 & 248 & 25.6 \\
6 & 7235 & 1738.14 & 6.9489 & 148 & 15.3 \\
7 & 7242 & 1736.01 & 6.9403 & 158 & 16.4 \\
8 & 7269 & 1727.77 & 6.9074 & 173 & 18.0 \\
9 & 7275 & 1725.94 & 6.9001 & 180 & 18.6 \\
10 & 7554 & 1640.79 & 6.5597 & 274 & 28.4 \\
11 & 7560 & 1638.96 & 6.5524 & 277 & 28.7 \\
12 & 7993 & 1506.82 & 6.0241 & 256 & 26.5 \\
13 & 8027 & 1496.45 & 5.9826 & 246 & 25.4 \\
14 & 8670 & 1300.22 & 5.1981 & 186 & 19.2 \\
15 & 8688 & 1294.72 & 5.1761 & 222 & 23.0 \\
16 & 8702 & 1290.45 & 5.1591 & 236 & 24.4 \\
17 & 8720 & 1284.96 & 5.1371 & 232 & 24.0 \\
18 & 9275 & 1115.59 & 4.4600 & 226 & 23.4 \\
19 & 9294 & 1109.79 & 4.4368 & 228 & 23.6 \\
20 & 9302 & 1107.35 & 4.4270 & 280 & 29.0 \\
21 & 9320 & 1101.85 & 4.4051 & 246 & 25.5 \\
22 & 9451 & 1061.88 & 4.2452 & 228 & 23.6 \\
23 & 9481 & 1052.72 & 4.2086 & 313 & 32.4 \\
24 & 9510 & 1043.87 & 4.1733 & 186 & 19.2 \\
\hline
\end{tabular}

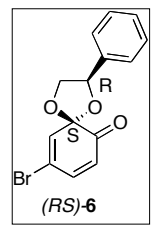




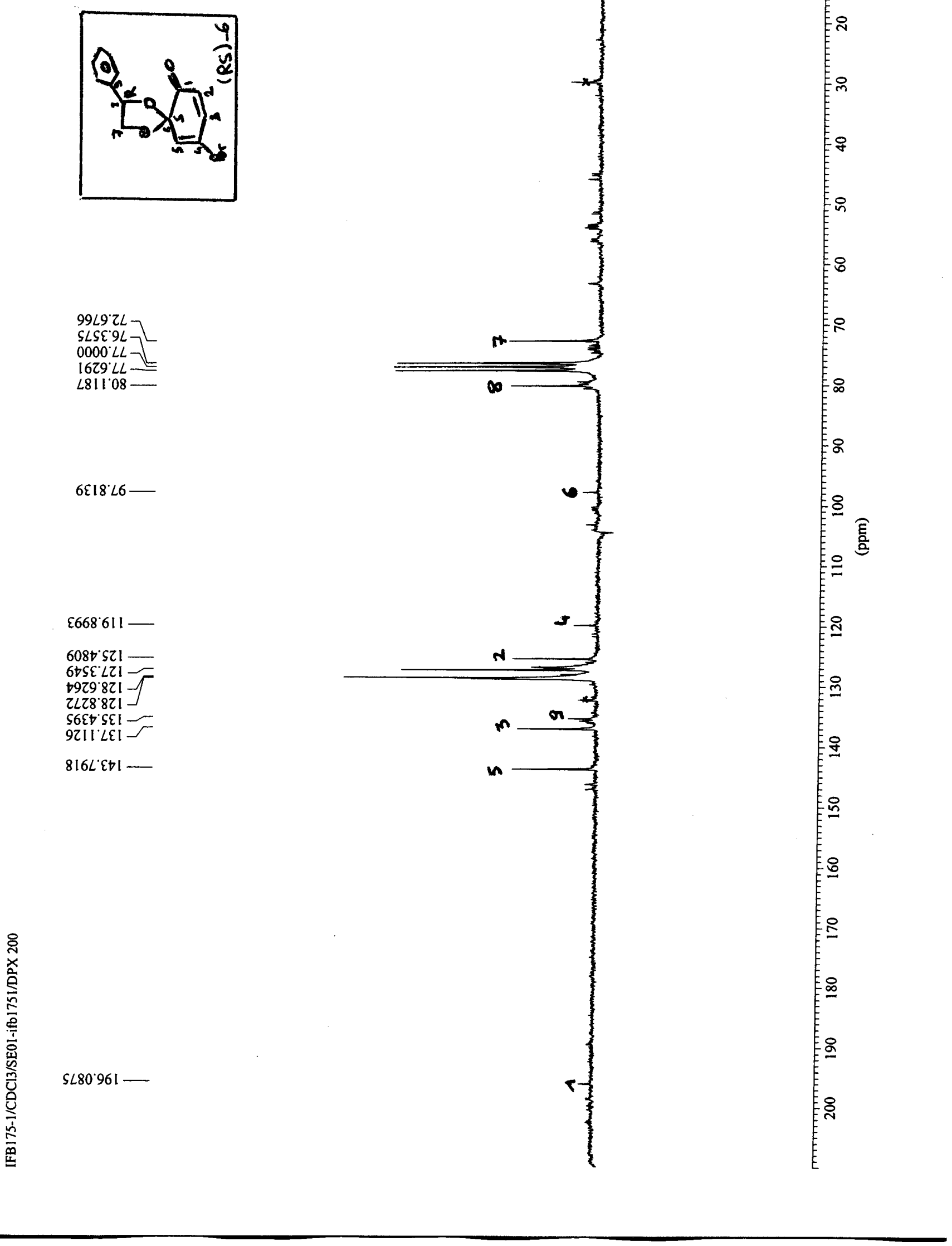



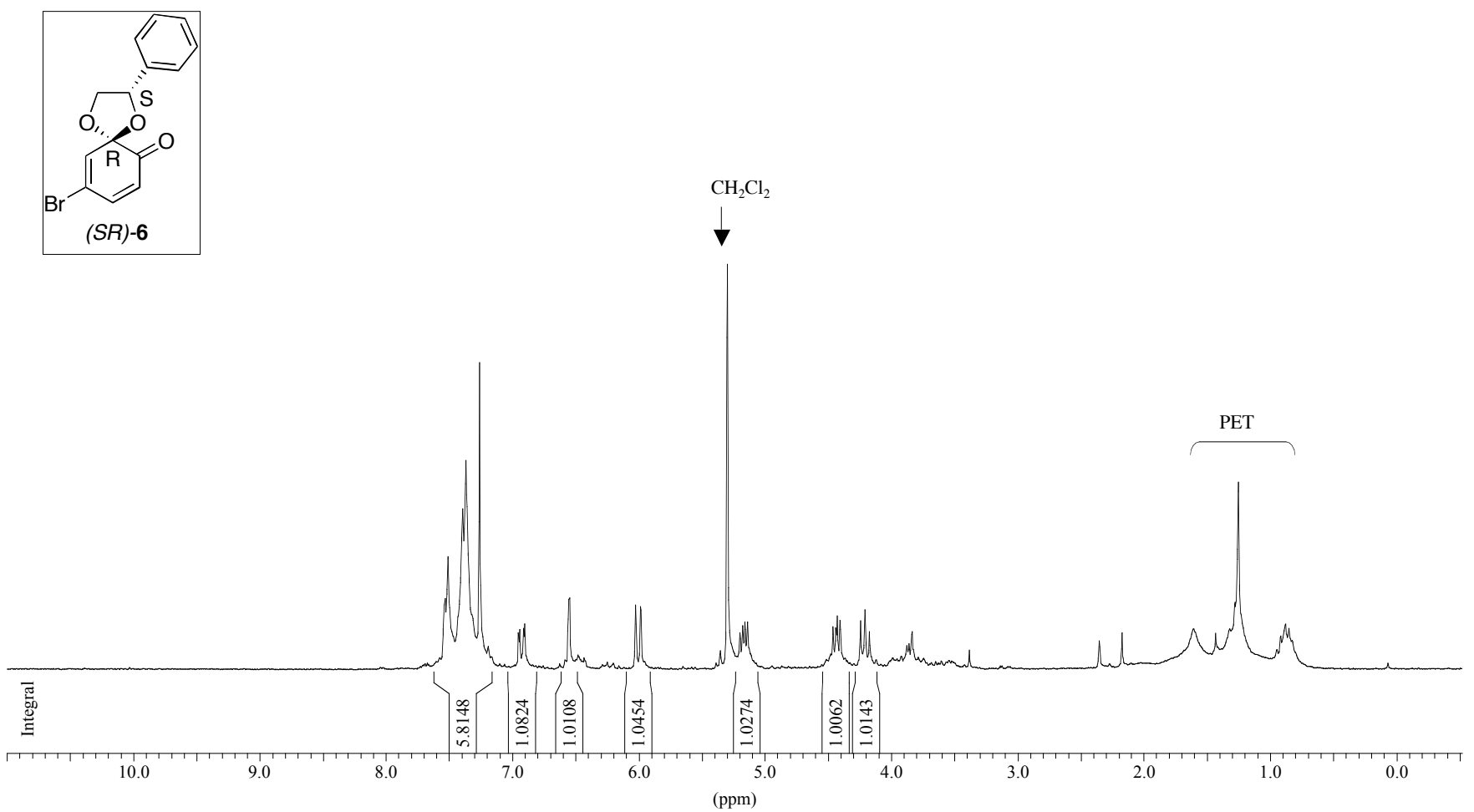

\begin{tabular}{crrrrr}
\hline Peak Nr. & Data Point & Frequency & PPM & Intensity & \%lnt. \\
\hline 1 & 6756 & 1884.02 & 7.5321 & 328 & 23.0 \\
2 & 6775 & 1878.22 & 7.5089 & 520 & 36.6 \\
3 & 6869 & 1849.53 & 7.3942 & 744 & 52.3 \\
4 & 6892 & 1842.51 & 7.3661 & 969 & 68.1 \\
5 & 6979 & 1815.96 & 7.2600 & 1423 & 100.0 \\
6 & 7231 & 1739.06 & 6.9525 & 165 & 11.6 \\
7 & 7239 & 1736.62 & 6.9428 & 181 & 12.7 \\
8 & 7265 & 1728.68 & 6.9111 & 197 & 13.9 \\
9 & 7272 & 1726.55 & 6.9025 & 205 & 14.4 \\
10 & 7556 & 1639.88 & 6.5560 & 323 & 22.7 \\
11 & 7563 & 1637.74 & 6.5475 & 328 & 23.1 \\
12 & 7990 & 1507.43 & 6.0265 & 298 & 20.9 \\
13 & 8024 & 1497.06 & 5.9850 & 289 & 20.3 \\
14 & 8671 & 1299.61 & 5.1957 & 166 & 11.7 \\
15 & 8689 & 1294.11 & 5.1737 & 199 & 14.0 \\
16 & 8703 & 1289.84 & 5.1566 & 217 & 15.2 \\
17 & 8721 & 1284.35 & 5.1347 & 215 & 15.1 \\
18 & 9274 & 1115.59 & 4.4600 & 194 & 13.6 \\
19 & 9292 & 1110.09 & 4.4380 & 188 & 13.2 \\
20 & 9301 & 1107.35 & 4.4270 & 246 & 17.3 \\
21 & 9319 & 1101.85 & 4.4051 & 223 & 15.7 \\
22 & 9451 & 1061.57 & 4.2440 & 220 & 15.5 \\
23 & 9482 & 1052.11 & 4.2062 & 275 & 19.3 \\
24 & 9510 & 1043.56 & 4.1720 & 171 & 12.0 \\
\hline
\end{tabular}

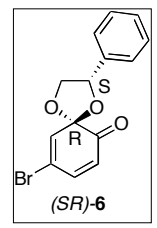


$S+80^{\circ} 9 L$

$0000^{\circ} L$

t00S $\angle L-L$

L8EI'08 -

$\angle 8+8^{\circ} \angle 6$

S016.6II-

9SOS SZI -

90LE'LZI-F

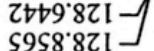

¿ZS† $\triangle \varepsilon I-\digamma$

tSEI LEI -

$0 \angle 08^{\circ} \varepsilon \mathrm{DI}-$

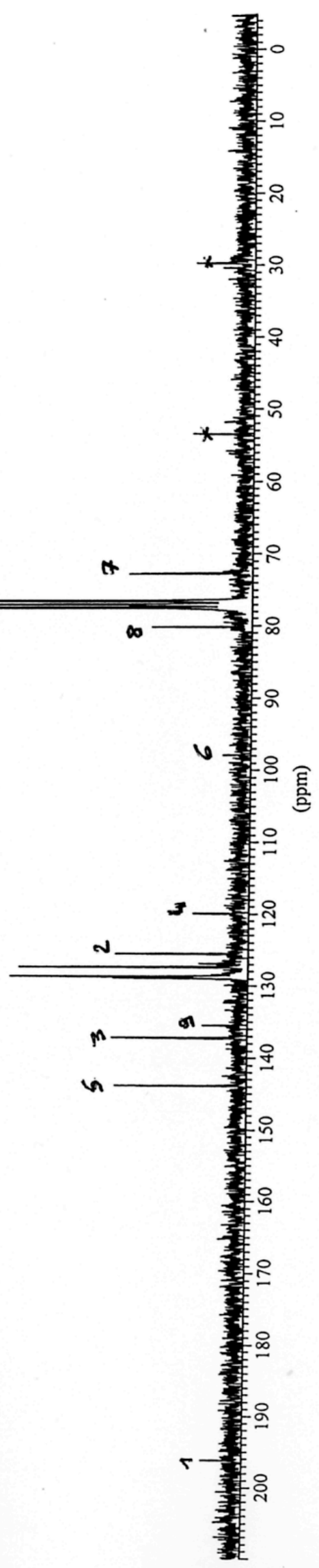




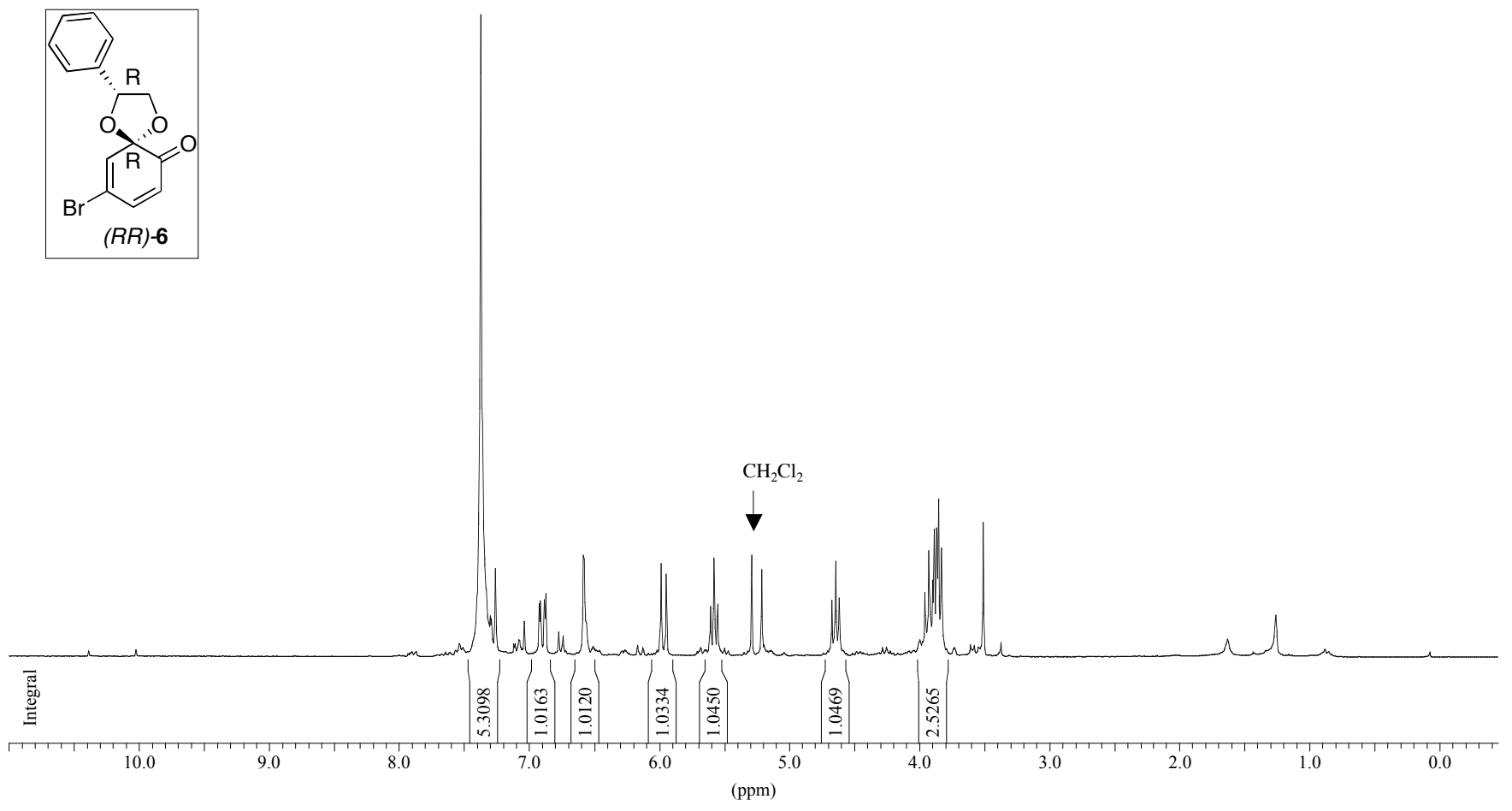

\begin{tabular}{|c|c|c|c|c|c|c|}
\hline Peak Nr. & Data Point & Frequency & PPM & Intensity & $\%$ int. & \\
\hline 1 & 6887 & 1844.04 & 7.3722 & 2013 & 100.0 & \\
\hline 2 & 6979 & 1815.96 & 7.2600 & 275 & 13.6 & \\
\hline 3 & 7255 & 1731.74 & 6.9233 & 163 & 8.1 & \\
\hline 4 & 7262 & 1729.60 & 6.9147 & 173 & 8.6 & \\
\hline 5 & 7288 & 1721.67 & 6.8830 & 176 & 8.7 & \\
\hline 6 & 7296 & 1719.22 & 6.8732 & 195 & 9.7 & \\
\hline 7 & 7533 & 1646.90 & 6.5841 & 317 & 15.7 & \\
\hline 8 & 7540 & 1644.76 & 6.5755 & 302 & 15.0 & \\
\hline 9 & 8021 & 1497.97 & 5.9887 & 290 & 14.4 & \\
\hline 10 & 8055 & 1487.60 & 5.9472 & 257 & 12.8 & \\
\hline 11 & 8333 & 1402.76 & 5.6080 & 154 & 7.7 & \\
\hline 12 & 8355 & 1396.04 & 5.5812 & 308 & 15.3 & \\
\hline 13 & 8378 & 1389.02 & 5.5531 & 160 & 8.0 & \\
\hline 14 & 9100 & 1168.69 & 4.6723 & 175 & 8.7 & \\
\hline 15 & 9124 & 1161.36 & 4.6430 & 297 & 14.7 & \\
\hline 16 & 9148 & 1154.04 & 4.6137 & 176 & 8.7 & (RR)-6 \\
\hline 17 & 9686 & 989.85 & 3.9573 & 200 & 9.9 & \\
\hline 18 & 9710 & 982.53 & 3.9280 & 331 & 16.5 & \\
\hline 19 & 9719 & 979.78 & 3.9170 & 145 & 7.2 & \\
\hline 20 & 9734 & 975.21 & 3.8987 & 238 & 11.8 & \\
\hline 21 & 9744 & 972.15 & 3.8865 & 399 & 19.8 & \\
\hline 22 & 9745 & 971.85 & 3.8853 & 391 & 19.4 & \\
\hline 23 & 9760 & 967.27 & 3.8670 & 402 & 20.0 & \\
\hline 24 & 9772 & 963.61 & 3.8524 & 482 & 23.9 & \\
\hline 25 & 9790 & 958.12 & 3.8304 & 340 & 16.9 & \\
\hline
\end{tabular}




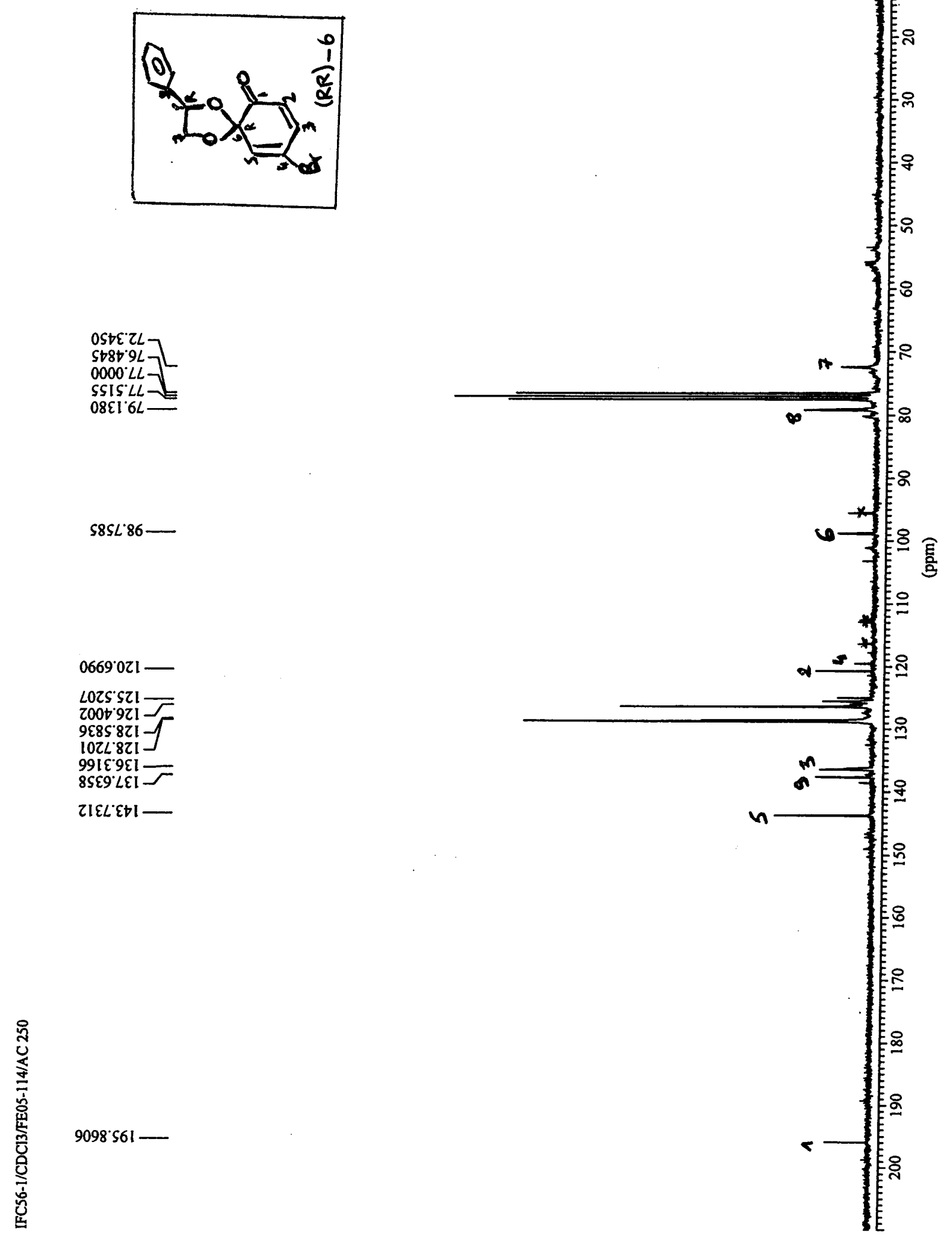



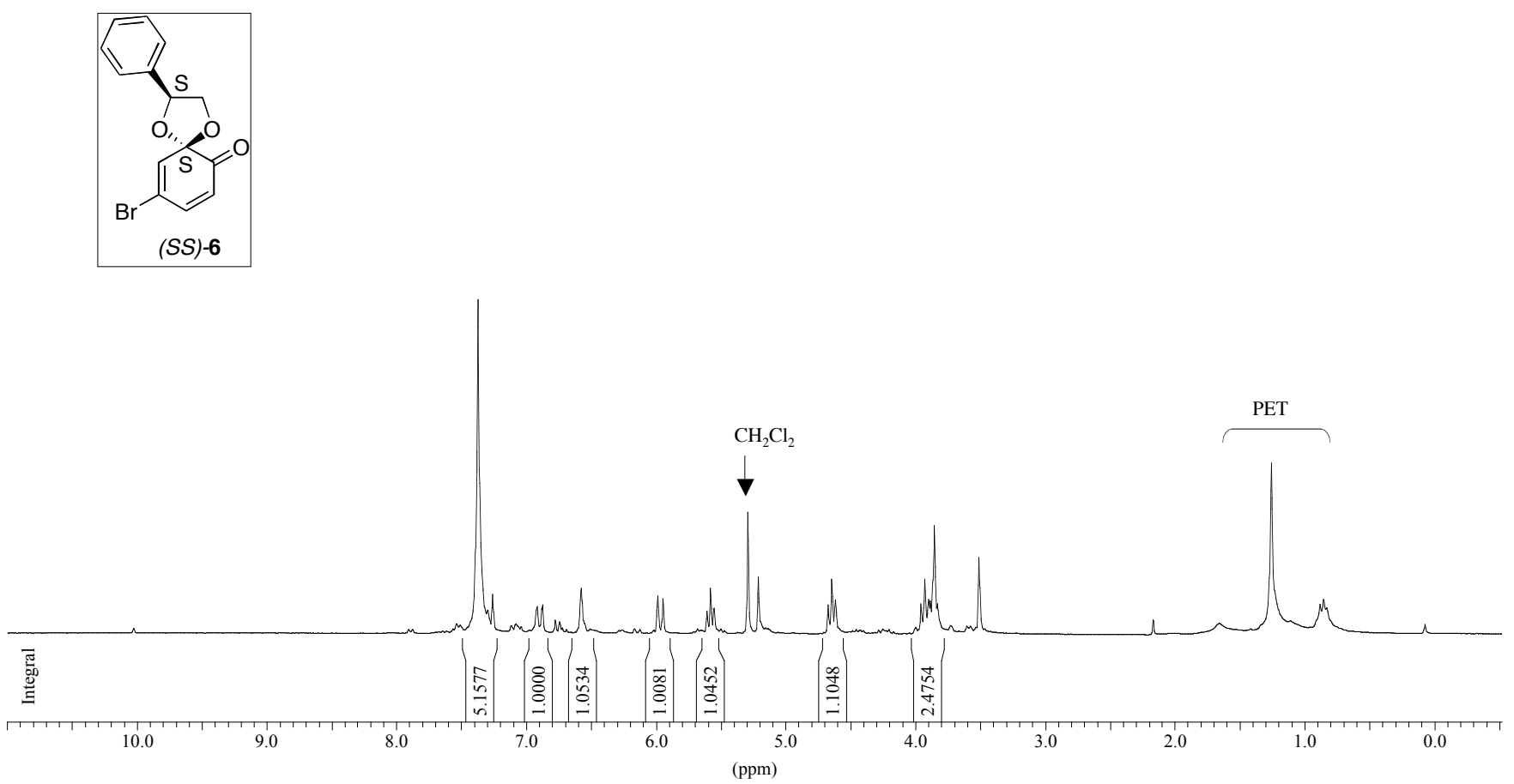

\begin{tabular}{ccrlrr}
\hline Peak Nr. & Data Point & Frequency & PPM & Intensity & \%lnt. \\
\hline 1 & 6887 & 1844.04 & 7.3722 & 2168 & 100.0 \\
2 & 6947 & 1825.73 & 7.2990 & 150 & 6.9 \\
3 & 6953 & 1823.90 & 7.2917 & 149 & 6.9 \\
4 & 6979 & 1815.96 & 7.2600 & 254 & 11.7 \\
5 & 7254 & 1732.04 & 6.9245 & 152 & 7.0 \\
6 & 7261 & 1729.90 & 6.9159 & 175 & 8.1 \\
7 & 7288 & 1721.67 & 6.8830 & 161 & 7.4 \\
8 & 7295 & 1719.53 & 6.8745 & 186 & 8.6 \\
9 & 7534 & 1646.59 & 6.5829 & 271 & 12.5 \\
10 & 7539 & 1645.07 & 6.5768 & 296 & 13.6 \\
11 & 8021 & 1497.97 & 5.9887 & 244 & 11.3 \\
12 & 8054 & 1487.90 & 5.9484 & 226 & 10.4 \\
13 & 8332 & 1403.06 & 5.6093 & 147 & 6.8 \\
14 & 8354 & 1396.35 & 5.5824 & 295 & 13.6 \\
15 & 8377 & 1389.33 & 5.5544 & 163 & 7.5 \\
16 & 9099 & 1168.99 & 4.6735 & 186 & 8.6 \\
17 & 9123 & 1161.67 & 4.6442 & 355 & 16.4 \\
18 & 9147 & 1154.34 & 4.6149 & 217 & 10.0 \\
19 & 9685 & 990.16 & 3.9585 & 195 & 9.0 \\
20 & 9709 & 982.83 & 3.9293 & 356 & 16.4 \\
21 & 9733 & 975.51 & 3.9000 & 221 & 10.2 \\
22 & 9744 & 972.15 & 3.8865 & 210 & 9.7 \\
23 & 9771 & 963.91 & 3.8536 & 701 & 32.3 \\
24 & 9789 & 958.42 & 3.8316 & 194 & 9.0 \\
\hline
\end{tabular}

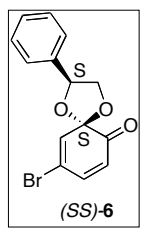




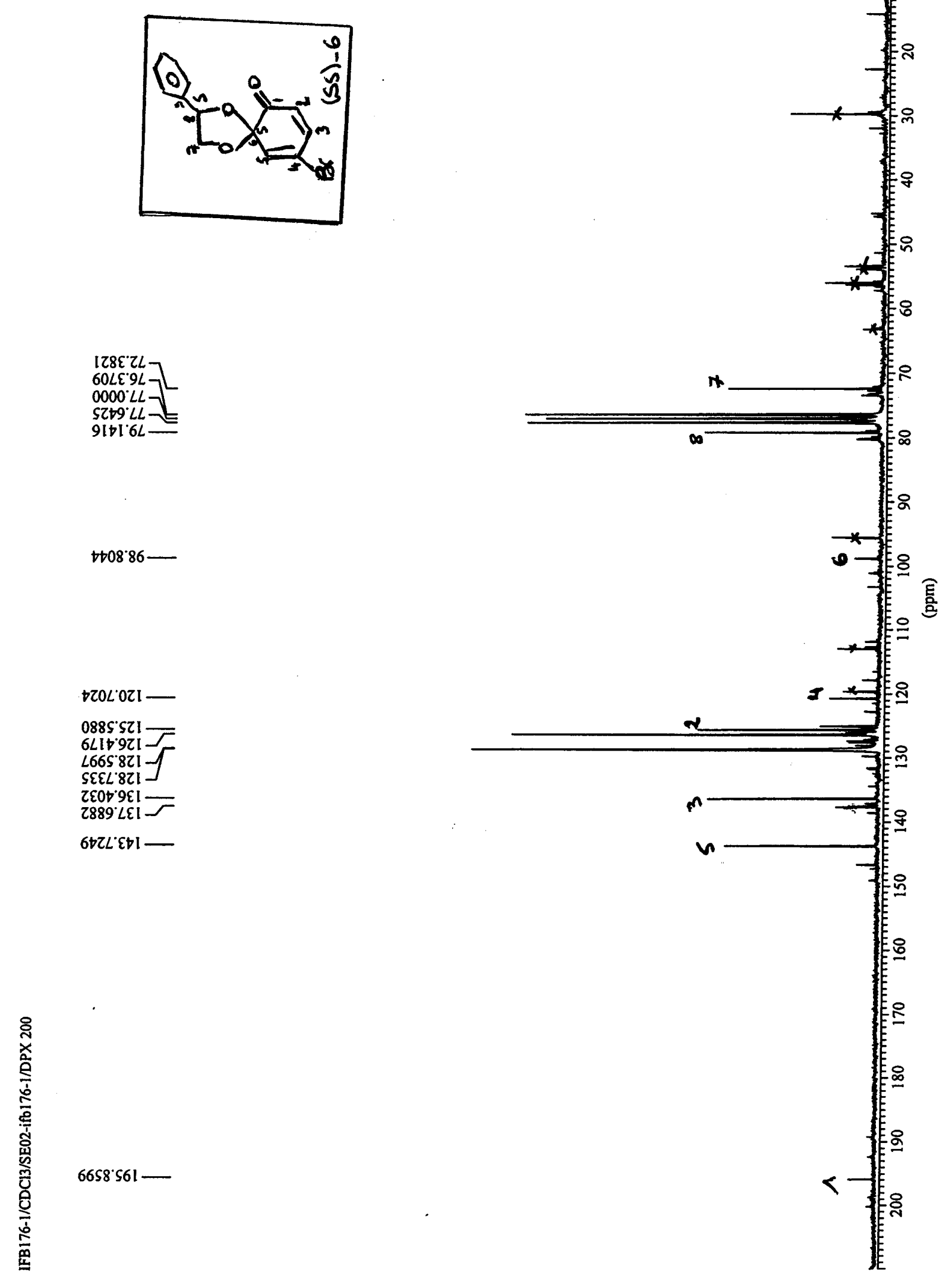




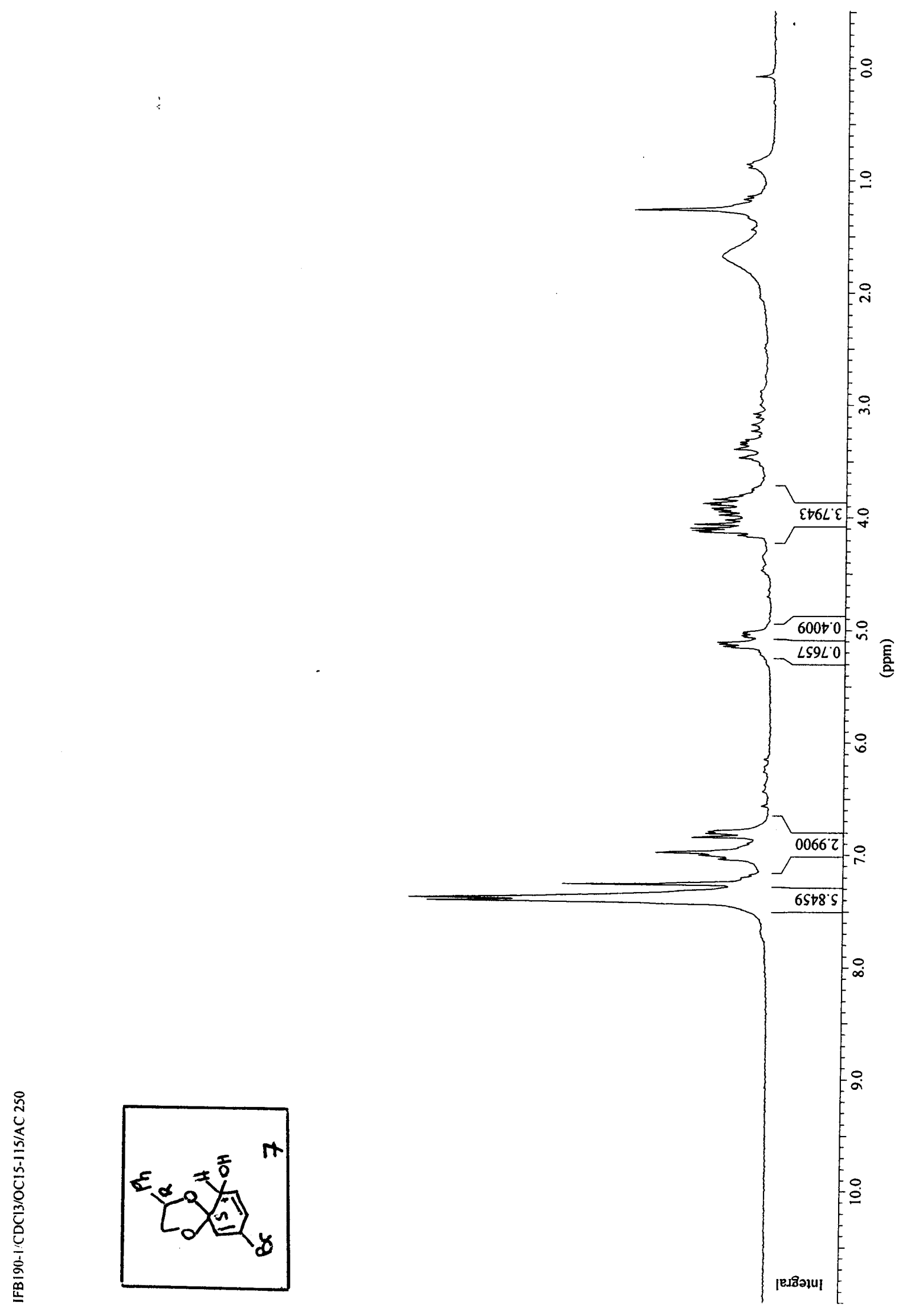


File Name C $: \backslash W I N 1 D \backslash S P C \backslash A S P 3000 \backslash O C 15 \backslash 115001.1 \mathrm{R}$

Peak Results saved in File: Nucleus : $1 \mathrm{H}$ SF OFFSET $S W \_p$ : $250.132854 \mathrm{MHz}$

: $15.7760 \mathrm{ppm}$ : $5000.00 \mathrm{~Hz}$ : 16384

Peak Picking Parameter

$\begin{array}{llr}\text { Peak constant PC } & = & 1.00 \\ \text { Noise } & = & 1 \\ \text { Sens. level } & = & 5\end{array}$

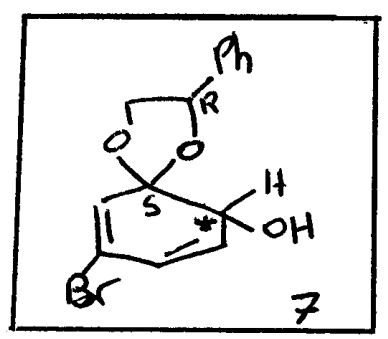

Peak Picking region

\begin{tabular}{rrrr} 
Start $(\mathrm{ppm} / \mathrm{Hz})$ & End $(\mathrm{ppm} / \mathrm{Hz})$ & MI $\left(\frac{\%}{8}\right)$ & MAXI $(\%)$ \\
\hline $7.47 / 1869.1$ & $7.06 / 1765.6$ & 43.73 & 106.64 \\
$7.14 / 1786.1$ & $6.69 / 1673.4$ & 11.09 & 39.41 \\
$5.22 / 1305.7$ & $4.94 / 1236.4$ & 5.77 & 16.57 \\
$4.40 / 1100.3$ & $3.74 / 936.4$ & 7.25 & 30.38 \\
\hline
\end{tabular}

Peak Picking results

\begin{tabular}{|c|c|c|c|c|c|}
\hline Peak & Nr. Data Point & Frequency & PPM & Intensity & sint. \\
\hline 1 & 6862 & 1851.98 & 7.4040 & 1049 & 94.9 \\
\hline 2 & 6883 & 1845.57 & 7.3783 & 1106 & 100.0 \\
\hline 3 & 6980 & 1815.96 & 7.2600 & 632 & 57.1 \\
\hline 4 & 7163 & 1760.12 & 7.0367 & 156 & 14.1 \\
\hline 5 & 7192 & 1751.27 & 7.0013 & 215 & 19.5 \\
\hline 6 & 7211 & 1745.47 & 6.9782 & 348 & 31.4 \\
\hline 7 & 7322 & 1711.59 & 6.8427 & 238 & 21.5 \\
\hline 8 & 7350 & 1703.05 & 6.8086 & 195 & 17.6 \\
\hline 9 & 7363 & 1699.08 & 6.7927 & 188 & 17.0 \\
\hline 10 & 8706 & 1289.23 & 5.1542 & 122 & 11.0 \\
\hline 11 & 8716 & 1286.18 & 5.1420 & 147 & 13.3 \\
\hline 12 & 8733 & 1280.99 & 5.1212 & 159 & 14.4 \\
\hline 13 & 8744 & 1277.63 & 5.1078 & 164 & 14.8 \\
\hline 14 & 8784 & 1265.43 & .5 .0590 & 82 & 7.5 \\
\hline 15 & 8795 & 1262.07 & 5.0456 & 90 & 8.2 \\
\hline 16 & 8809 & 1257.80 & 5.0285 & 87 & 7.9 \\
\hline 17 & 8820 & 1254.44 & 5.0151 & 85 & 7.7 \\
\hline 18 & 9515 & 1042.34 & 4.1672 & 82 & 7.4 \\
\hline 19 & 9525 & 1039.29 & 4.1550 & 106 & 9.6 \\
\hline 20 & 9548 & 1032.27 & 4.1269 & 224 & 20.3 \\
\hline 21 & 9558 & 1029.22 & 4.1147 & 246 & 22.3 \\
\hline 22 & 9576 & 1023.73 & 4.0927 & 251 & 22.7 \\
\hline 23 & 9604 & 1015.18 & 4.0586 & 238 & 21.6 \\
\hline 24 & 9637 & 1005.11 & 4.0183 & 144 & 13.1 \\
\hline 25 & 9671 & 994.74 & 3.9768 & 164 & 14.8 \\
\hline 26 & 9696 & 987.11 & 3.9463 & 165 & 14.9 \\
\hline 27 & 9710 & 982.83 & 3.9293 & 178 & 16.1 \\
\hline 28 & 9722 & 979.17 & 3.9146 & 186 & 16.8 \\
\hline 29 & 9745 & 972.15 & 3.8865 & 190 & 17.2 \\
\hline 30 & 9758 & 968.19 & 3.8707 & 214 & 19.3 \\
\hline 31 & 9789 & 958.73 & 3.8329 & 182 & 16.5 \\
\hline 32 & 9821 & 948.96 & 3.7938 & 102 & 9.2 \\
\hline
\end{tabular}




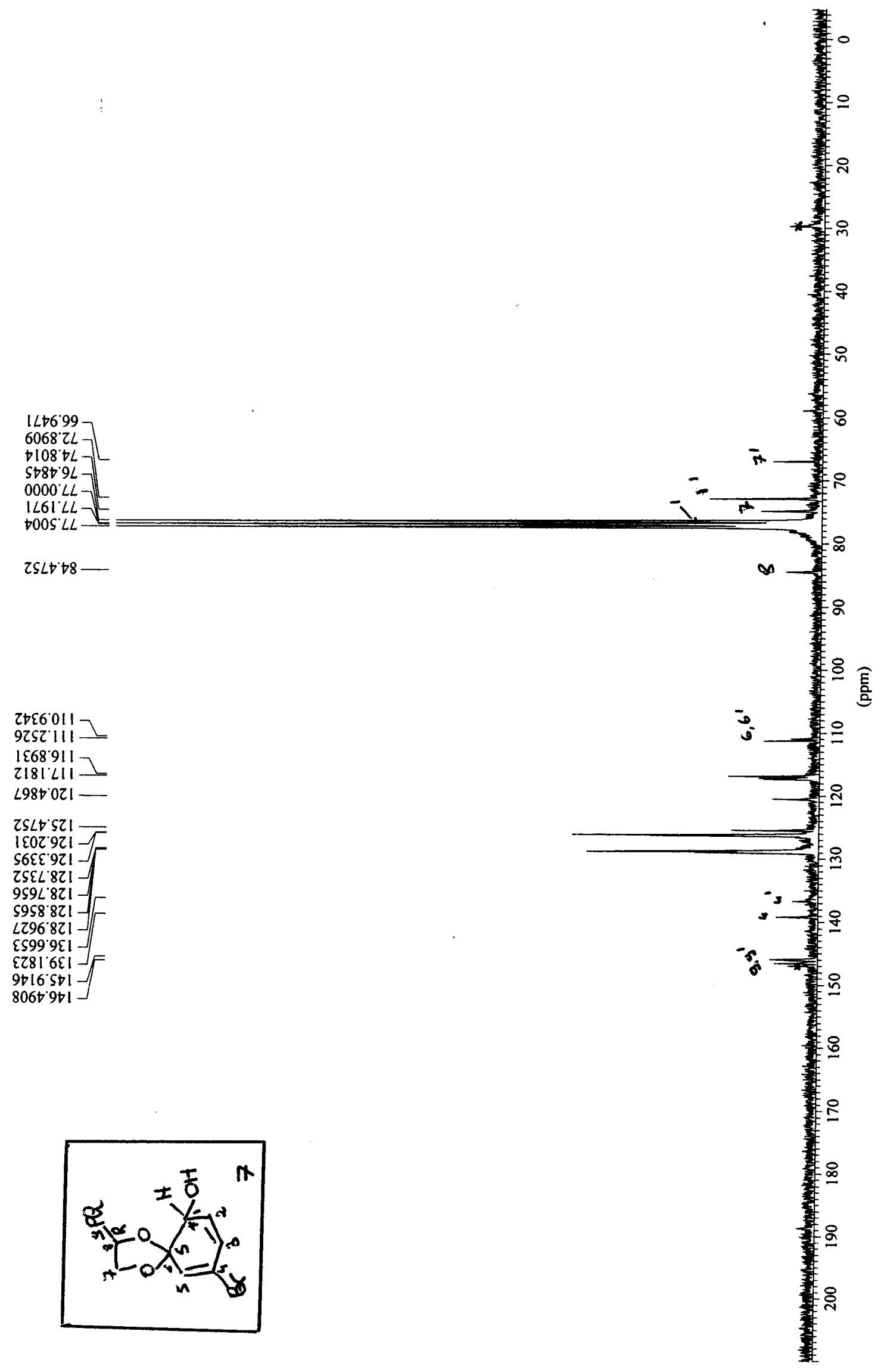




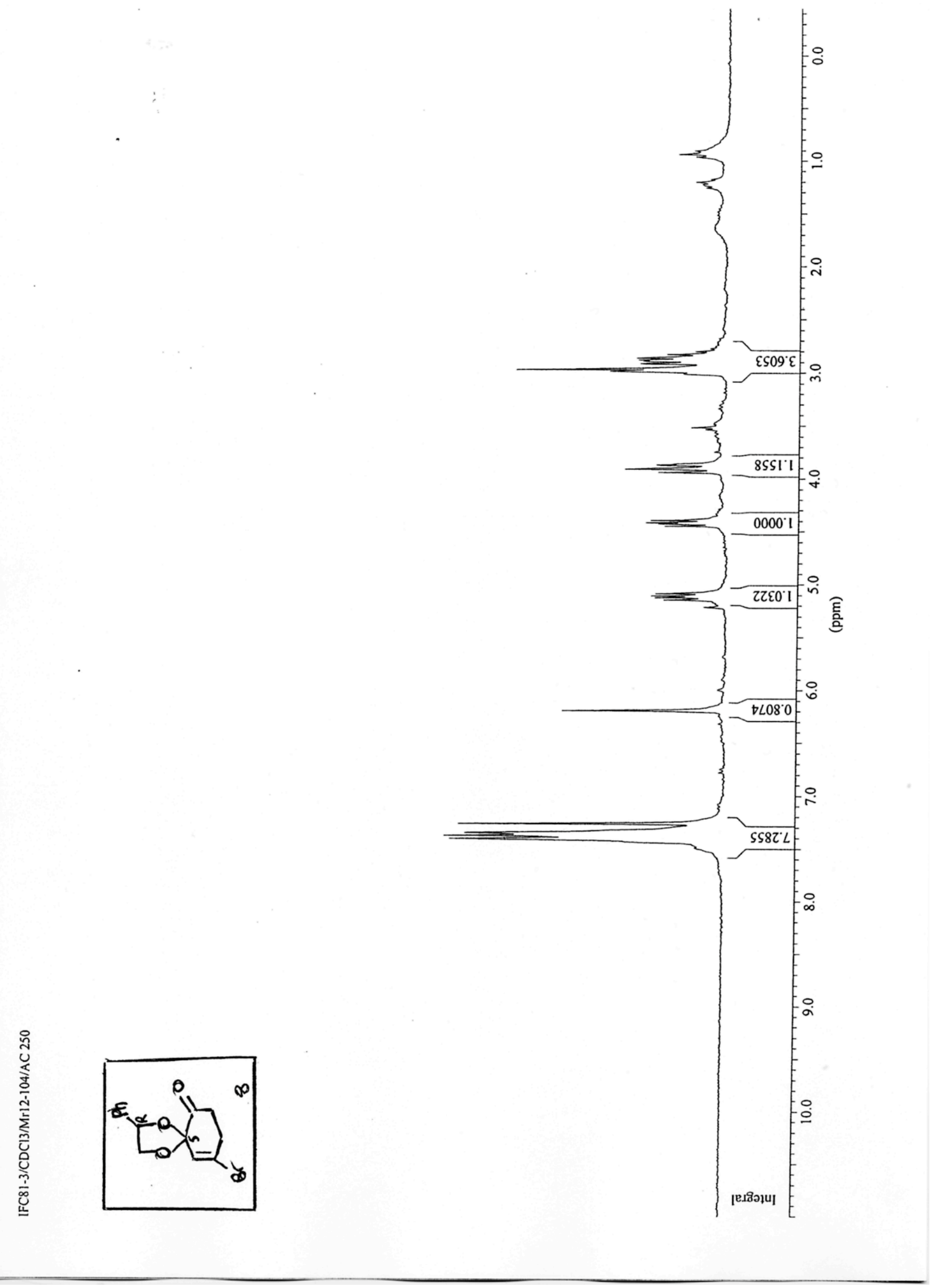


File Name

Peak Results saved in File:

Nucleus

SF

OFFSET

SW_P

$S I$

Peak Picking Parameter

Peak constant PC =

Noise

Sens. level
$C: \backslash W I N 1 D \backslash S P C \backslash A S P 3000 \backslash M R 120 F \backslash 104001.1 R$

$1 \mathrm{H}$

$250.132854 \mathrm{MHz}$

$15.7748 \mathrm{ppm}$

$5000.00 \mathrm{~Hz}$

16384

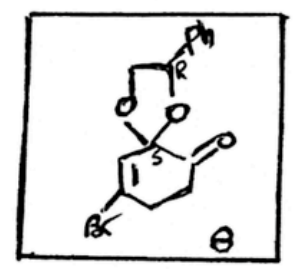

1.00

7

28

Peak Picking region

\begin{tabular}{lrrr} 
Start $(\mathrm{ppm} / \mathrm{Hz})$ & End $(\mathrm{ppm} / \mathrm{Hz})$ & MI (\%) & MAXI (\%) \\
\hline $15.77 / 3945.8$ & $-4.21 /-1054.2$ & -0.98 & 100.00 \\
\hline
\end{tabular}

Peak Picking results

\begin{tabular}{rrrrrr} 
Peak Nr. Data Point & Frequency & PPM & Intensity & \%Int. \\
\hline 1 & 6862 & 1851.67 & 7.4027 & 4710 & 98.0 \\
2 & 6888 & 1843.74 & 7.3710 & 4807 & 100.0 \\
3 & 6909 & 1837.33 & 7.3454 & 4441 & 92.4 \\
4 & 6979 & 1815.96 & 7.2600 & 4562 & 94.9 \\
5 & 7856 & 1548.33 & 6.1900 & 2783 & 57.9 \\
6 & 8716 & 1285.87 & 5.1408 & 1066 & 22.2 \\
7 & 8734 & 1280.38 & 5.1188 & 1211 & 25.2 \\
8 & 8747 & 1276.41 & 5.1029 & 1273 & 26.5 \\
9 & 8766 & 1270.62 & 5.0798 & 1205 & 25.1 \\
10 & 9286 & 1111.92 & 4.4453 & 1063 & 22.1 \\
11 & 9305 & 1106.13 & 4.4222 & 1171 & 24.4 \\
12 & 9314 & 1103.38 & 4.4112 & 1379 & 28.7 \\
13 & 9332 & 1097.89 & 4.3892 & 1298 & 27.0 \\
14 & 9702 & 984.97 & 3.9378 & 1180 & 24.5 \\
15 & 9732 & 975.82 & 3.9012 & 1743 & 36.3 \\
16 & 9762 & 966.66 & 3.8646 & 1208 & 25.1 \\
17 & 9768 & 964.83 & 3.8573 & 940 & 19.5 \\
18 & 10466 & 751.82 & 3.0057 & 756 & 15.7 \\
19 & 10488 & 745.10 & 2.9788 & 1989 & 41.4 \\
20 & 10505 & 739.91 & 2.9581 & 3618 & 75.3 \\
21 & 10546 & 727.40 & 2.9081 & 1489 & 31.0 \\
22 & 10567 & 720.99 & 2.8824 & 1426 & 29.7 \\
23 & 10574 & 718.86 & 2.8739 & 1519 & 31.6 \\
24 & 10590 & 713.97 & 2.8544 & 1553 & 32.3 \\
25 & 10615 & 706.35 & 2.8239 & 1033 & 21.5 \\
26 & 10636 & 699.94 & 2.7983 & 547 & 11.4 \\
\hline
\end{tabular}




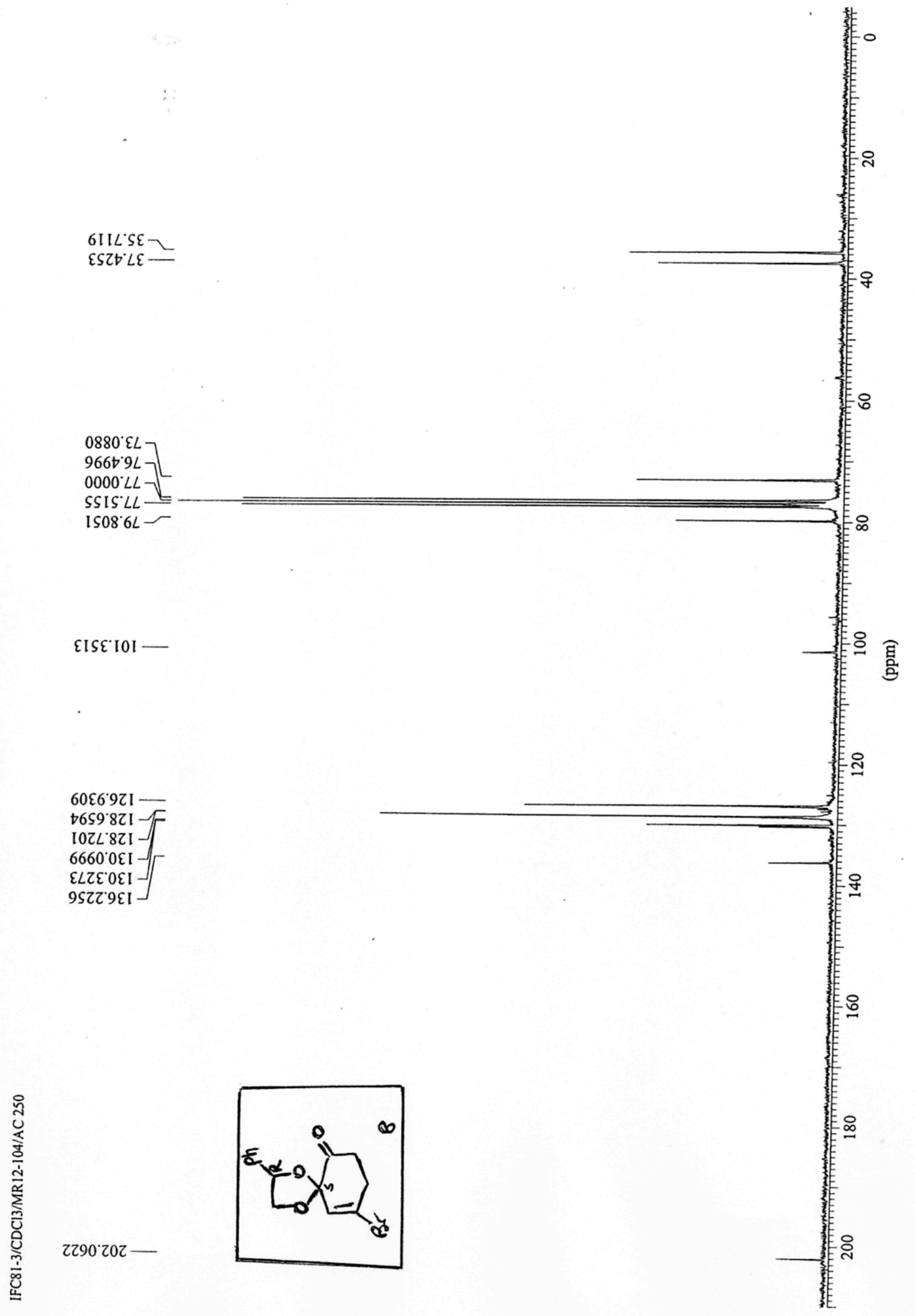



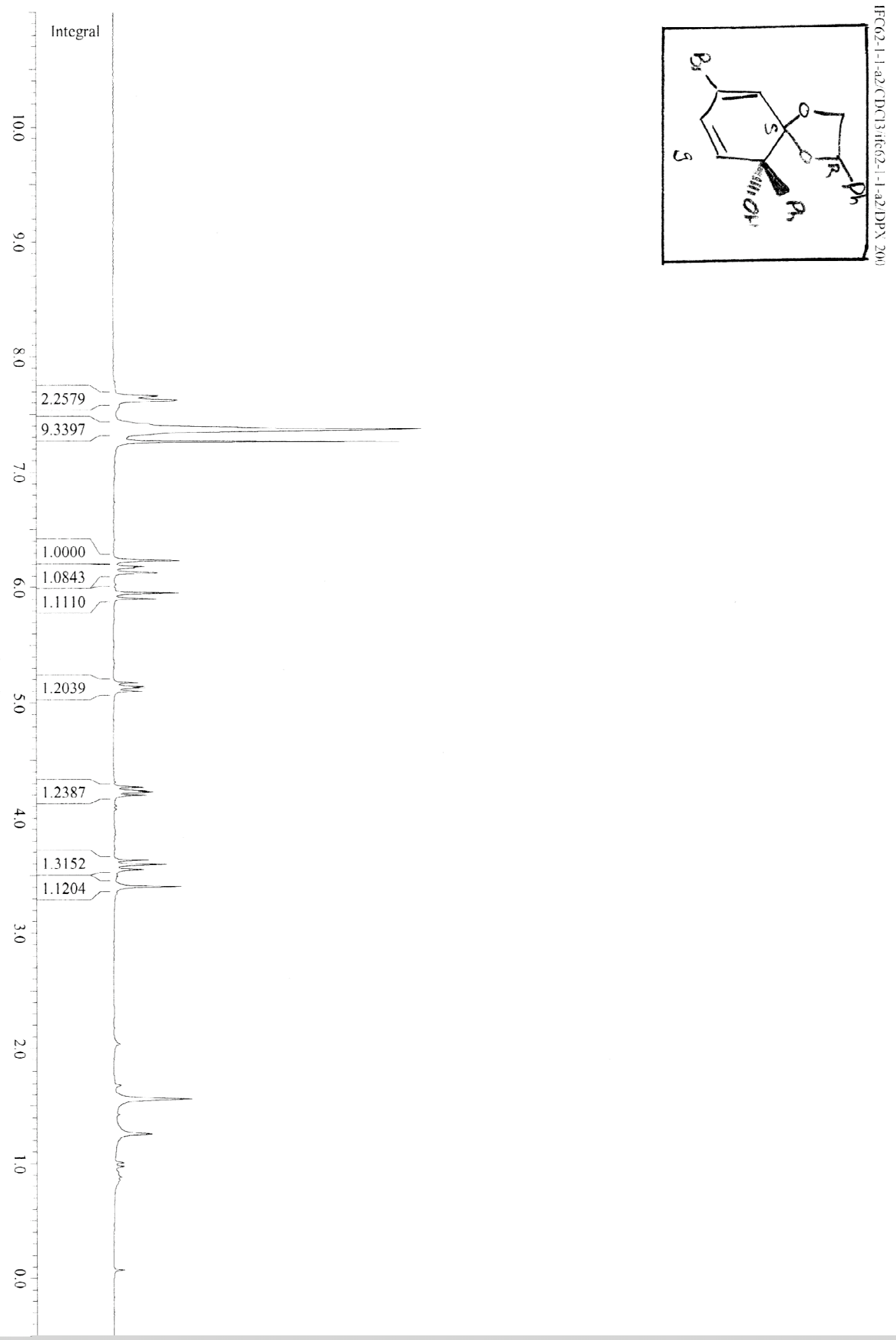
File Name

Peak Results saved in File:

Nucleus

$\mathrm{SF}$

OFFSET

SW_P

$S I$

Peak Picking Parameter

$\begin{array}{llr}\text { Peak constant PC } & = & 1.00 \\ \text { Noise level } & = & 259 \\ \text { Sens. level } & = & 1037\end{array}$

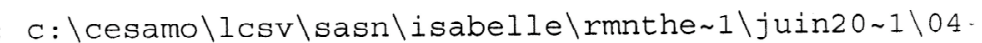

\section{off}

$200.160019 \mathrm{MHz}$

$11.9086 \mathrm{ppm}$

$2604.17 \mathrm{~Hz}$

16384

Peak Picking region

\begin{tabular}{crrr} 
Start $(\mathrm{ppm} / \mathrm{Hz})$ & End $(\mathrm{ppm} / \mathrm{Hz})$ & MI $(\%)$ & MAXI $(\%)$ \\
\hline $7.91 / 1583.3$ & $7.58 / 1517.2$ & 11.54 & 33.66 \\
$7.45 / 1490.2$ & $7.08 / 1416.9$ & 57.59 & 107.26 \\
$6.28 / 1257.3$ & $6.21 / 1242.2$ & 16.16 & 26.82 \\
$6.21 / 1242.7$ & $6.07 / 1214.3$ & 6.71 & 16.16 \\
$6.00 / 1201.7$ & $5.84 / 1168.5$ & 5.31 & 25.21 \\
$5.25 / 1051.8$ & $4.95 / 991.4$ & 6.31 & 18.58 \\
$4.29 / 858.9$ & $4.16 / 833.0$ & 6.31 & 16.77 \\
$3.72 / 745.5$ & $3.52 / 703.6$ & 5.71 & 24.41 \\
$3.46 / 692.1$ & $3.36 / 673.4$ & 16.97 & 25.42 \\
\hline
\end{tabular}

Peak picking results

\begin{tabular}{crrrrr} 
Peak Nr. Data Point & Frequency & PPM & Intensity & \% Int. \\
\hline 1 & 5348 & 1533.59 & 7.6618 & 25623 & 14.6 \\
2 & 5360 & 1531.68 & 7.6523 & 25303 & 14.4 \\
3 & 5398 & 1525.64 & 7.6221 & 36690 & 20.9 \\
4 & 5408 & 1524.05 & 7.6142 & 34147 & 19.5 \\
5 & 5717 & 1474.94 & 7.3688 & 175287 & 100.0 \\
6 & 5854 & 1453.16 & 7.2600 & 162455 & 92.7 \\
7 & 7144 & 1248.12 & 6.2356 & 32676 & 18.6 \\
8 & 7154 & 1246.53 & 6.2277 & 37850 & 21.6 \\
9 & 7214 & 1237.00 & 6.1800 & 17546 & 10.0 \\
10 & 7225 & 1235.25 & 6.1713 & 14226 & 8.1 \\
11 & 7277 & 1226.98 & 6.1300 & 25350 & 14.5 \\
12 & 7288 & 1225.23 & 6.1213 & 23139 & 13.2 \\
13 & 7501 & 1191.38 & 5.9521 & 37195 & 21.2 \\
14 & 7564 & 1181.36 & 5.9021 & 24349 & 13.9 \\
15 & 8482 & 1035.45 & 5.1731 & 13989 & 8.0 \\
16 & 8521 & 1029.25 & 5.1422 & 17767 & 10.1 \\
17 & 8537 & 1026.71 & 5.1294 & 17944 & 10.2 \\
18 & 8576 & 1020.51 & 5.0985 & 16587 & 9.5 \\
19 & 9626 & 853.62 & 4.2647 & 17018 & 9.7 \\
20 & 9665 & 847.42 & 4.2337 & 19149 & 10.9 \\
21 & 9677 & 845.51 & 4.2242 & 22495 & 12.8 \\
22 & 9716 & 839.31 & 4.1932 & 18823 & 10.7 \\
23 & 10421 & 727.26 & 3.6334 & 19890 & 11.3 \\
24 & 10474 & 718.83 & 3.5913 & 30188 & 17.2 \\
25 & 10527 & 710.41 & 3.5492 & 17094 & 9.8 \\
26 & 10710 & 681.32 & 3.4039 & 38560 & 22.0 \\
\hline & & & & &
\end{tabular}




$$
F !
$$




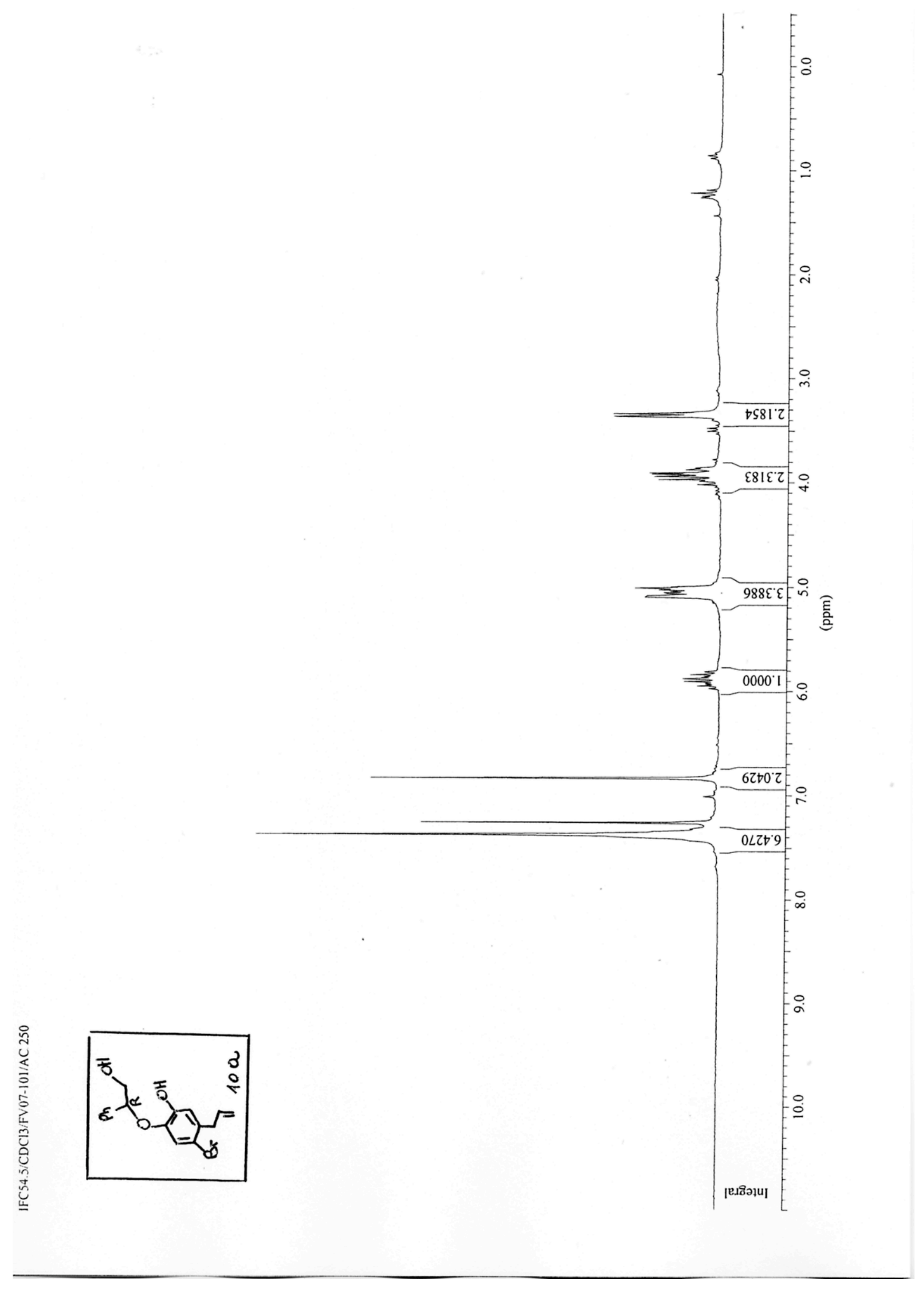




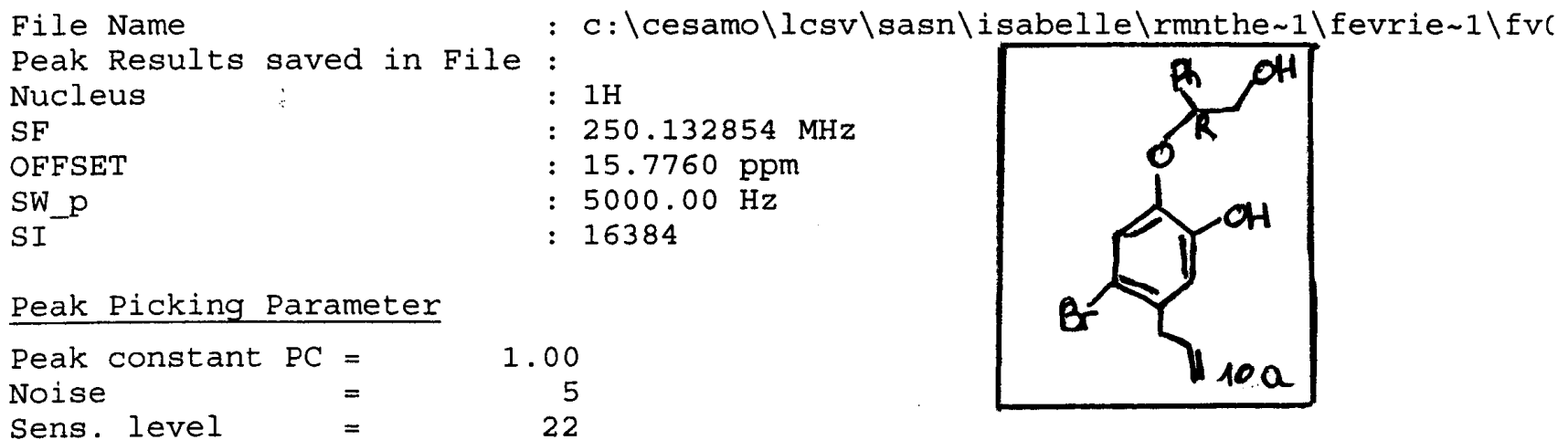

\section{Peak Picking region}

\begin{tabular}{rrrr} 
Start $(\mathrm{ppm} / \mathrm{Hz})$ & End $(\mathrm{ppm} / \mathrm{Hz})$ & MI $\left(\frac{o}{\gamma}\right)$ & MAXI $\left(\frac{q}{8}\right)$ \\
\hline $9.16 / 2292.3$ & $4.82 / 1206.5$ & 25.31 & 124.84 \\
$6.26 / 1565.4$ & $5.71 / 1427.8$ & 1.18 & 11.81 \\
$5.27 / 1319.1$ & $4.76 / 1190.0$ & 9.41 & 21.24 \\
$3.54 / 884.9$ & $3.11 / 778.4$ & 15.56 & 25.47 \\
$4.10 / 1024.6$ & $3.76 / 941.3$ & 3.89 & 16.44 \\
\hline
\end{tabular}

Peak Picking results

\begin{tabular}{rrrrrr} 
Peak Nr. Data Point & Frequency & \multicolumn{1}{c}{ PPM } & Intensity & \&Int. \\
\hline 1 & 6887 & 1844.35 & 7.3735 & 13803 & 100.0 \\
2 & 6980 & 1815.96 & 7.2600 & 8884 & 64.4 \\
3 & 7329 & 1709.46 & 6.8342 & 10416 & 75.5 \\
4 & 8038 & 1493.09 & 5.9692 & 320 & 2.3 \\
5 & 8060 & 1486.37 & 5.9423 & 657 & 4.8 \\
6 & 8072 & 1482.71 & 5.9277 & 427 & 3.1 \\
7 & 8081 & 1479.97 & 5.9167 & 420 & 3.0 \\
8 & 8093 & 1476.30 & 5.9021 & 1062 & 7.7 \\
9 & 8115 & 1469.59 & 5.8752 & 1098 & 8.0 \\
10 & 8127 & 1465.93 & 5.8606 & 545 & 3.9 \\
11 & 8136 & 1463.18 & 5.8496 & 461 & 3.3 \\
12 & 8148 & 1459.52 & 5.8350 & 868 & 6.3 \\
13 & 8170 & 1452.81 & 5.8081 & 443 & 3.2 \\
14 & 8757 & 1273.67 & 5.0920 & 2242 & 16.2 \\
15 & 8760 & 1272.75 & 5.0883 & 2129 & 15.4 \\
16 & 8765 & 1271.23 & 5.0822 & 2172 & 15.7 \\
17 & 8770 & 1269.70 & 5.0761 & 1712 & 12.4 \\
18 & 8785 & 1265.12 & 5.0578 & 1583 & 11.5 \\
19 & 8790 & 1263.60 & 5.0517 & 1680 & 12.2 \\
20 & 8800 & 1260.54 & 5.0395 & 1473 & 10.7 \\
21 & 8813 & 1256.58 & 5.0236 & 1826 & 13.2 \\
22 & 8821 & 1254.14 & 5.0139 & 1988 & 14.4 \\
23 & 8826 & 1252.61 & 5.0078 & 2544 & 18.4 \\
24 & 8837 & 1249.25 & 4.9944 & 1465 & 10.6 \\
25 & 9640 & 1004.20 & 4.0147 & 697 & 5.0 \\
26 & 9666 & 996.26 & 3.9829 & 655 & 4.7 \\
27 & 9679 & 992.30 & 3.9671 & 1861 & 13.5 \\
28 & 9705 & 984.36 & 3.9354 & 1987 & 14.4 \\
29 & 9720 & 979.78 & 3.9171 & 2067 & 15.0 \\
30 & 9731 & 976.43 & 3.9036 & 2137 & 15.5 \\
31 & 9758 & 968.19 & 3.8707 & 1050 & 7.6
\end{tabular}




\begin{tabular}{rrrrrr}
2 & 9770 & 964.52 & 3.8560 & 764 & 5.5 \\
3 & 10178 & 840.01 & 3.3583 & 3191 & 23.1 \\
4 & 10200 & 833.30 & 3.3314 & 3214 & 23.3 \\
\hline
\end{tabular}




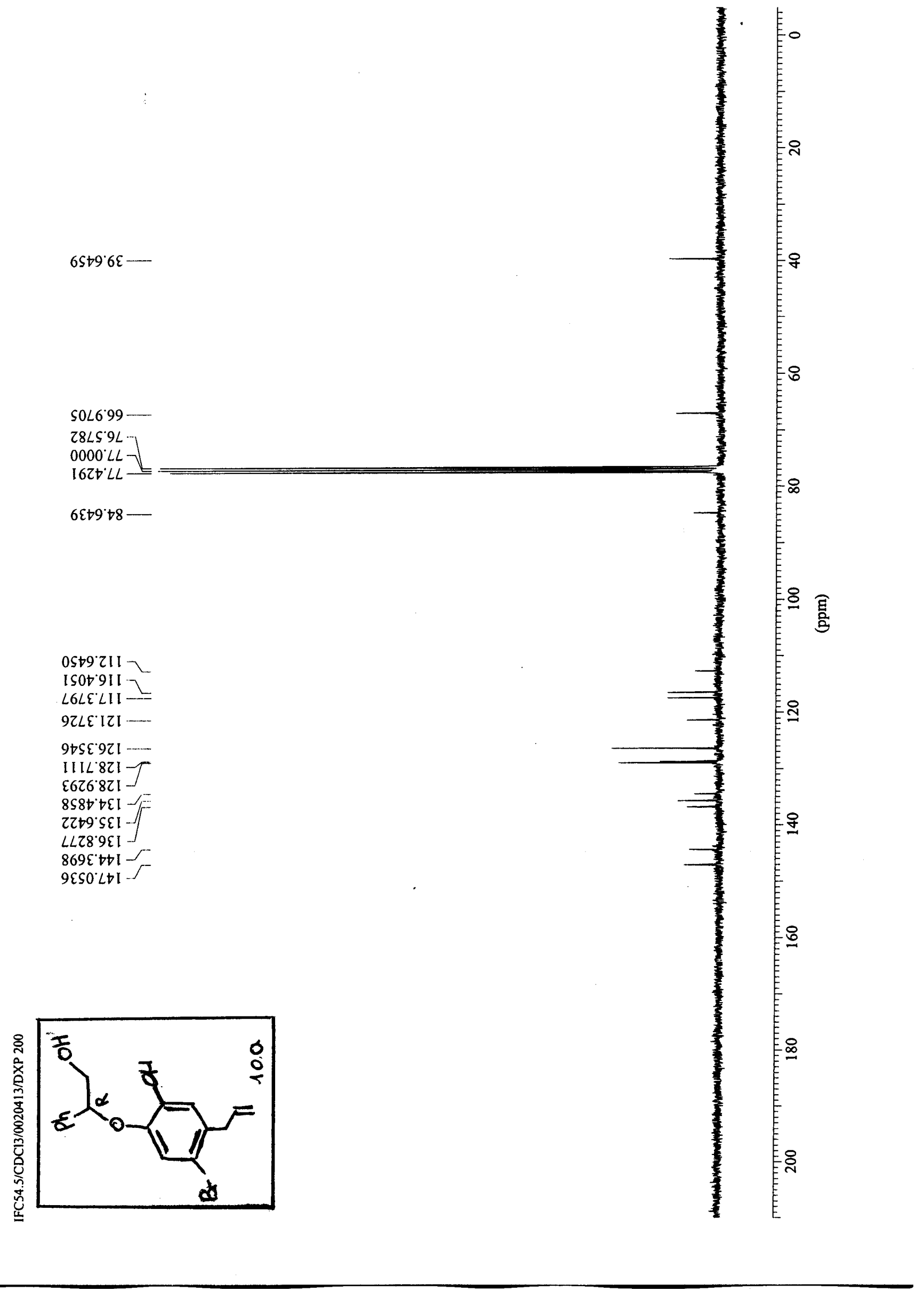




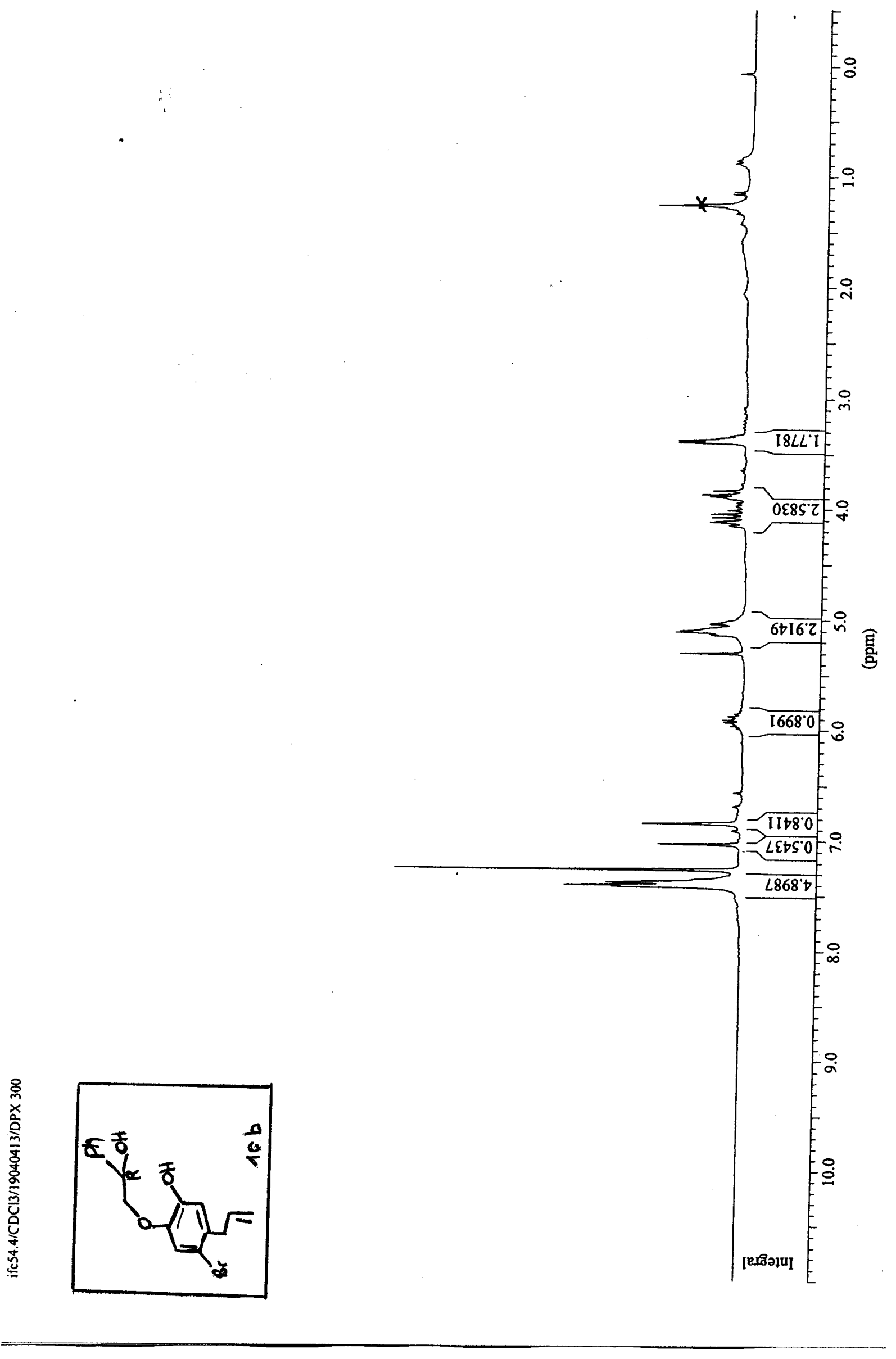


File Name

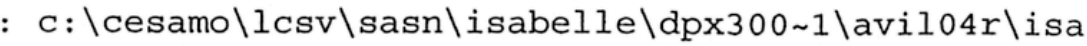

Peak Results saved in File:

Nucleus

$\mathrm{SF}$

: off

OFFSET

SW_p

: $300.130007 \mathrm{MHz}$

: $19.7618 \mathrm{ppm}$

: $6172.84 \mathrm{~Hz}$

SI : 16384

Peak Picking Parameter

Peak constant $\mathrm{PC}=$

1.00

Noise

330

Sens. level=

1318

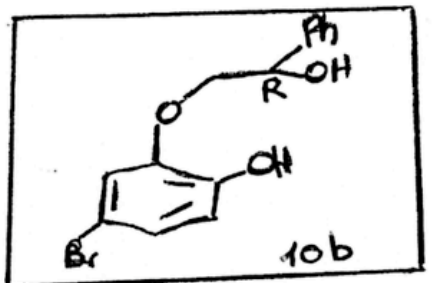

Peak Picking region

\begin{tabular}{rrrr} 
Start $(\mathrm{ppm} / \mathrm{Hz})$ & End $(\mathrm{ppm} / \mathrm{Hz})$ & MI $(\%)$ & MAXI (\%) \\
\hline $4.26 / 1277.7$ & $3.68 / 1104.1$ & 2.76 & 25.29 \\
$3.52 / 1056.9$ & $3.26 / 977.8$ & 14.61 & 25.44 \\
$4.03 / 1209.6$ & $4.01 / 1202.8$ & 21.49 & 22.95 \\
$7.53 / 2258.8$ & $7.24 / 2174.4$ & 27.28 & 57.57 \\
$7.13 / 2141.3$ & $6.69 / 2007.5$ & 11.61 & 39.60 \\
$6.11 / 1832.3$ & $5.73 / 1720.0$ & 2.16 & 11.64 \\
$5.15 / 1544.9$ & $4.91 / 1474.4$ & 7.23 & 23.87 \\
\hline
\end{tabular}

Peak Picking results

\begin{tabular}{rrrrrr} 
Peak Nr. Data Point & Frequency & \multicolumn{1}{c}{ PPM } & Intensity & \% Int. \\
1 & 9837 & 2224.91 & 7.4132 & 69063 & 37.4 \\
2 & 9848 & 2220.76 & 7.3993 & 94363 & 51.1 \\
3 & 9867 & 2213.61 & 7.3755 & 72171 & 39.1 \\
4 & 9959 & 2178.94 & 7.2600 & 184552 & 100.0 \\
5 & 10143 & 2109.62 & 7.0290 & 44872 & 24.3 \\
6 & 10292 & 2053.48 & 6.8420 & 53420 & 28.9 \\
7 & 10975 & 1796.16 & 5.9846 & 4372 & 2.4 \\
8 & 10992 & 1789.75 & 5.9633 & 7788 & 4.2 \\
9 & 11020 & 1779.20 & 5.9281 & 11069 & 6.0 \\
10 & 11037 & 1772.80 & 5.9068. & 11660 & 6.3 \\
11 & 11064 & 1762.62 & 5.8729 & 8996 & 4.9 \\
12 & 11081 & 1756.22 & 5.8515 & 5476 & 3.0 \\
13 & 11652 & 1541.09 & 5.1347 & 18494 & 10.0 \\
14 & 11660 & 1538.07 & 5.1247 & 19894 & 10.8 \\
15 & 11674 & 1532.80 & 5.1071 & 37834 & 20.5 \\
16 & 11684 & 1529.03 & 5.0946 & 35140 & 19.0 \\
17 & 11697 & 1524.13 & 5.0782 & 24996 & 13.5 \\
18 & 11731 & 1511.32 & 5.0356 & 19240 & 10.4 \\
19 & 12435 & 1246.09 & 4.1518 & 9675 & 5.2 \\
20 & 12443 & 1243.07 & 4.1418 & 10489 & 5.7 \\
21 & 12462 & 1235.91 & 4.1179 & 20232 & 11.0 \\
22 & 12470 & 1232.90 & 4.1079 & 18335 & 9.9 \\
23 & 12498 & 1222.35 & 4.0727 & 19385 & 10.5 \\
24 & 12521 & 1213.68 & 4.0439 & 19733 & 10.7 \\
25 & 12548 & 1203.51 & 4.0100 & 10952 & 5.9 \\
26 & 12572 & 1194.47 & 3.9798 & 6366 & 3.4 \\
27 & 12581 & 1191.08 & 3.9685 & 6894 & 3.7 \\
28 & 12596 & 1185.43 & 3.9497 & 5634 & 3.1 \\
29 & 12603 & 1182.79 & 3.9409 & 6480 & 3.5
\end{tabular}




\begin{tabular}{rrrrrr}
30 & 12630 & 1172.62 & 3.9070 & 10243 & 5.6 \\
31 & 12640 & 1168.85 & 3.8945 & 12825 & 6.9 \\
32 & 12646 & 1166.59 & 3.8869 & 20610 & 11.2 \\
33 & 12660 & 1161.31 & 3.8694 & 25254 & 13.7 \\
34 & 12689 & 1150.39 & 3.8330 & 19122 & 10.4 \\
35 & 13036 & 1019.65 & 3.3974 & 37469 & 20.3 \\
36 & 13041 & 1017.77 & 3.3911 & 37706 & 20.4 \\
37 & 13052 & 1013.62 & 3.3773 & 37820 & 20.5 \\
\hline
\end{tabular}




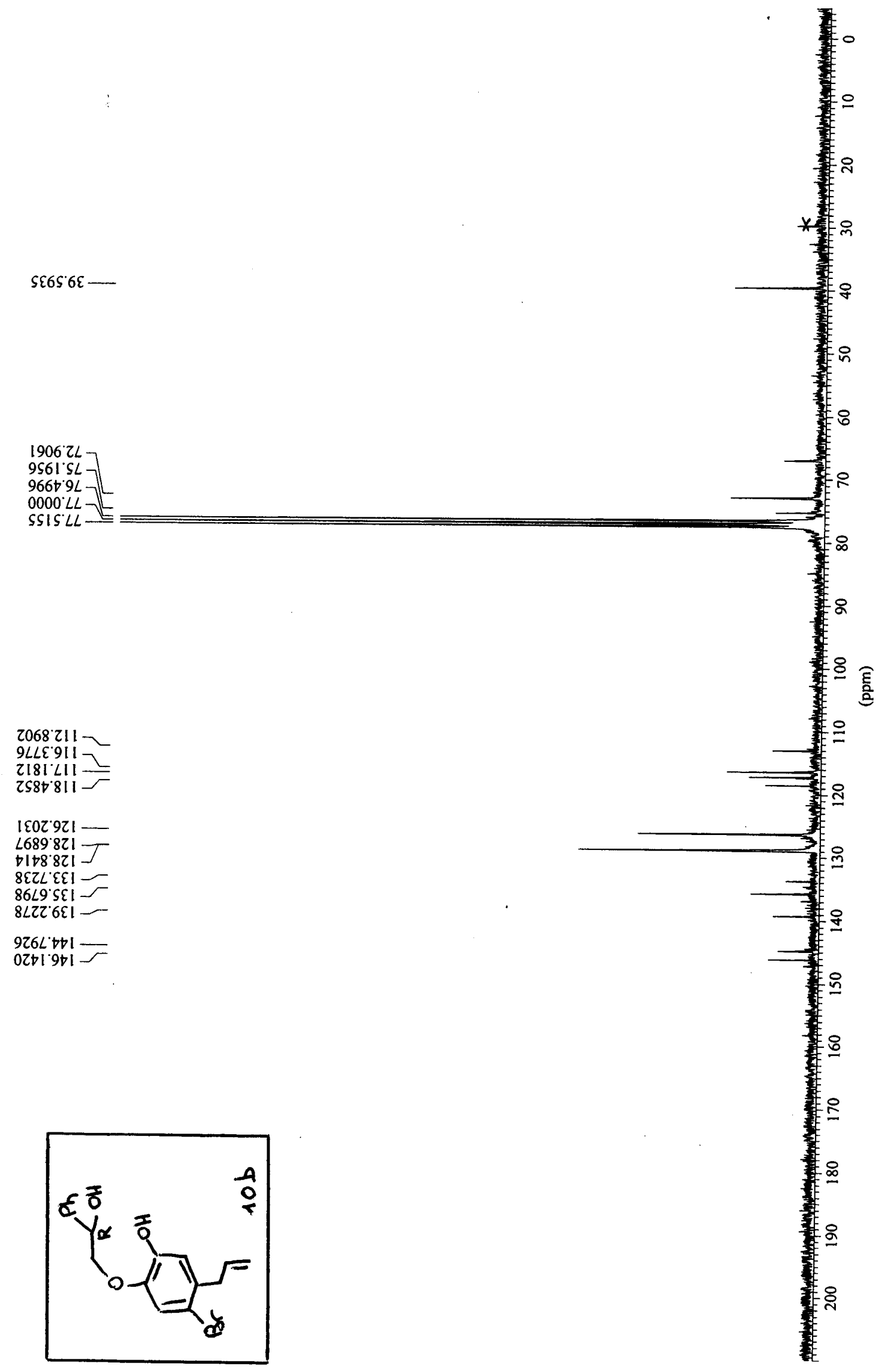



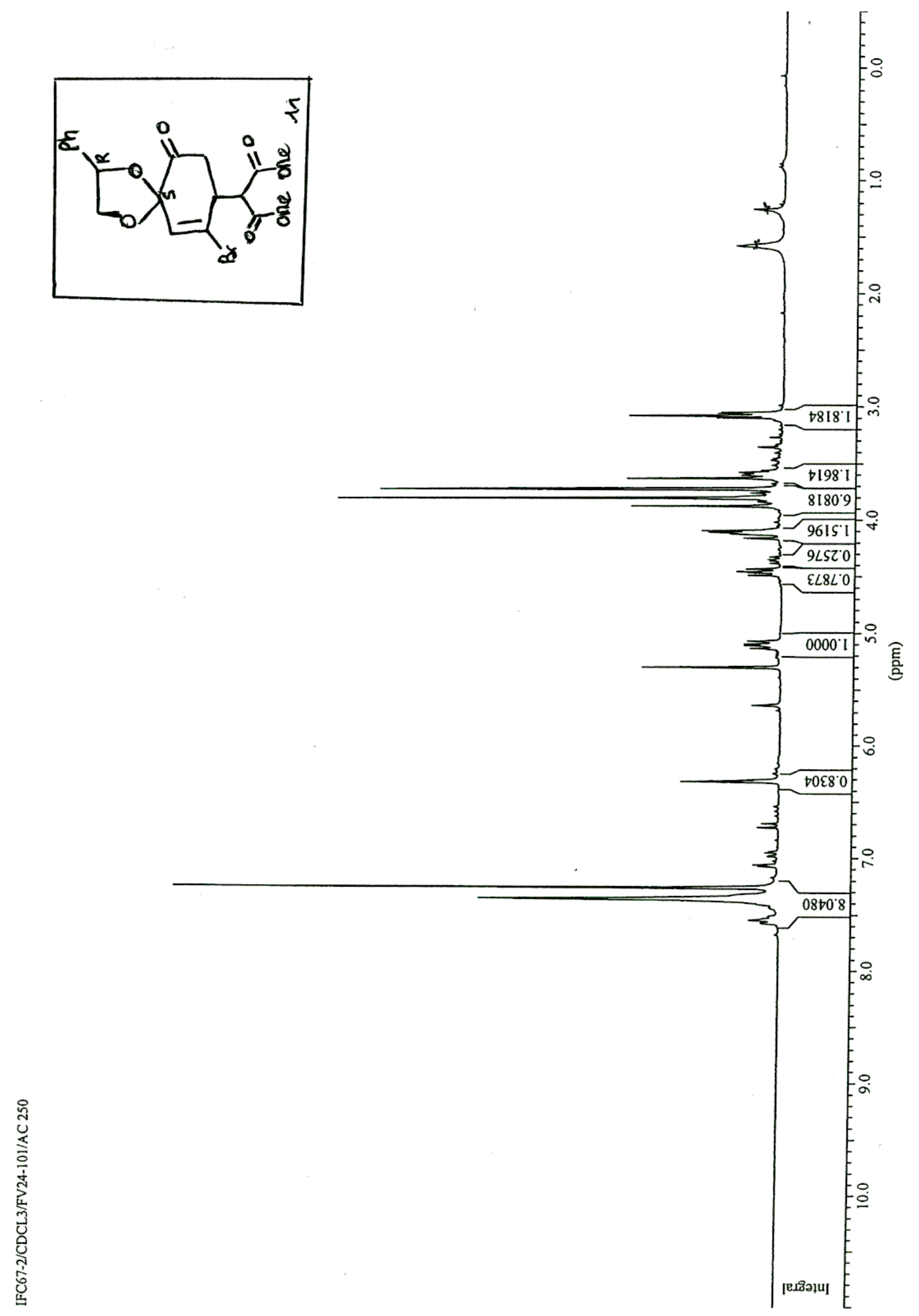
File Name

Peak Results saved in File

Nucleus

SF

OFFSET

SW_p

$S I^{-}$

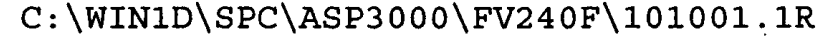

: $1 \mathrm{H}$

: $250.132854 \mathrm{MHz}$

: $15.7748 \mathrm{ppm}$

$5000.00 \mathrm{~Hz}$

16384

Peak Picking Parameter

Peak constant $\mathrm{PC}=$

Noise

Sens. level

1.00

$=7$

26

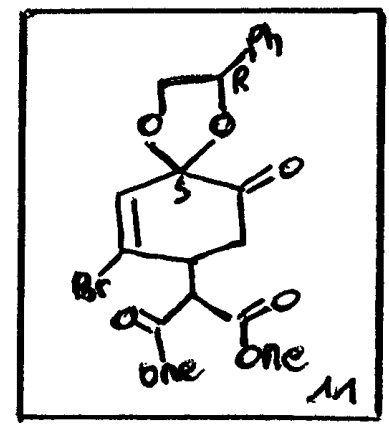

Peak Picking region

\begin{tabular}{|c|c|c|c|c|c|}
\hline \multicolumn{2}{|c|}{ Start (ppm/Hz) } & \multicolumn{2}{|c|}{ End (ppm/Hz) } & MI $\left(\frac{\gamma}{8}\right)$ & $\operatorname{MAXI}(8)$ \\
\hline $7.37 /$ & 1844.7 & $7.17 /$ & 1794.0 & 80.35 & 109.98 \\
\hline $7.44 /$ & 1860.8 & $7.28 /$ & 1821.8 & 35.06 & 57.07 \\
\hline $7.61 /$ & 1903.2 & $7.51 /$ & 1877.9 & 1.83 & 8.18 \\
\hline $6.40 \%$ & 1601.1 & $6.21 /$ & 1553.8 & 3.95 & 20.24 \\
\hline $5.21 /$ & 1304.2 & $4.94 /$ & 1235.8 & 3.81 & 10.14 \\
\hline $5.38 /$ & 1346.3 & $5.23 /$ & 1307.2 & 22.21 & 26.04 \\
\hline $4.51 /$ & 1128.4 & $4.33 /$ & 1084.2 & 3.91 & 9.47 \\
\hline $4.39 /$ & 1099.1 & $4.29 /$ & 1074.1 & 1.32 & 3.24 \\
\hline $3.96 /$ & 989.9 & $3.67 /$ & 918.4 & 58.94 & 85.77 \\
\hline
\end{tabular}

Peak Picking results

\begin{tabular}{rrrrrr} 
Peak Nr. Data Point & Frequency & \multicolumn{1}{c}{ PPM } & Intensity & \% Int. \\
1 & 6718 & 1895.62 & 7.5784 & 772 & 2.7 \\
2 & 6725 & 1893.48 & 7.5699 & 839 & 2.9 \\
3 & 6744 & 1887.68 & 7.5467 & 1390 & 4.8 \\
4 & 6749 & 1886.15 & 7.5406 & 1339 & 4.6 \\
5 & 6900 & 1840.07 & 7.3564 & 14379 & 49.6 \\
6 & 6979 & 1815.96 & 7.2600 & 28999 & 100.0 \\
7 & 7750 & 1580.67 & 6.3193 & 4719 & 16.3 \\
8 & 7761 & 1577.32 & 6.3059 & 2023 & 7.0 \\
9 & 8586 & 1325.55 & 5.2994 & 6653 & 22.9 \\
10 & 8724 & 1283.43 & 5.1310 & 1490 & 5.1 \\
11 & 8743 & 1277.63 & 5.1078 & 1760 & 6.1 \\
12 & 8756 & 1273.67 & 5.0920 & 1760 & 6.1 \\
13 & 8775 & 1267.87 & 5.0688 & 1618 & 5.6 \\
14 & 9252 & 1122.30 & 4.4868 & 1615 & 5.6 \\
15 & 9271 & 1116.50 & 4.4636 & 1615 & 5.6 \\
16 & 9279 & 1114.06 & 4.4539 & 2157 & 7.4 \\
17 & 9298 & 1108.26 & 4.4307 & 1716 & 5.9 \\
18 & 9339 & 1095.75 & 4.3807 & 555 & 1.9 \\
19 & 9358 & 1089.95 & 4.3575 & 575 & 2.0 \\
20 & 9367 & 1087.20 & 4.3465 & 676 & 2.3 \\
21 & 9385 & 1081.71 & 4.3245 & 597 & 2.1 \\
22 & 9553 & 1030.44 & 4.1196 & 2562 & 8.8 \\
23 & 9564 & 1027.09 & 4.1062 & 3523 & 12.1 \\
24 & 9576 & 1023.42 & 4.0915 & 3841 & 13.2 \\
25 & 9755 & 968.80 & 3.8731 & 7228 & 24.9 \\
26 & 9813 & 951.10 & 3.8024 & 21306 & 73.5 \\
27 & 9880 & 930.65 & 3.7206 & 19293 & 66.5
\end{tabular}




\begin{tabular}{rrrrrr}
$: 8$ & 9957 & 907.15 & 3.6267 & 7480 & 25.8 \\
19 & 10392 & 774.40 & 3.0960 & 3217 & 11.1 \\
10 & 10409 & 769.21 & 3.0752 & 7389 & 25.5 \\
11 & 10424 & 764.63 & 3.0569 & 3134 & 10.8 \\
$i 2$ & 10431 & 762.50 & 3.0484 & 2966 & 10.2 \\
\hline
\end{tabular}




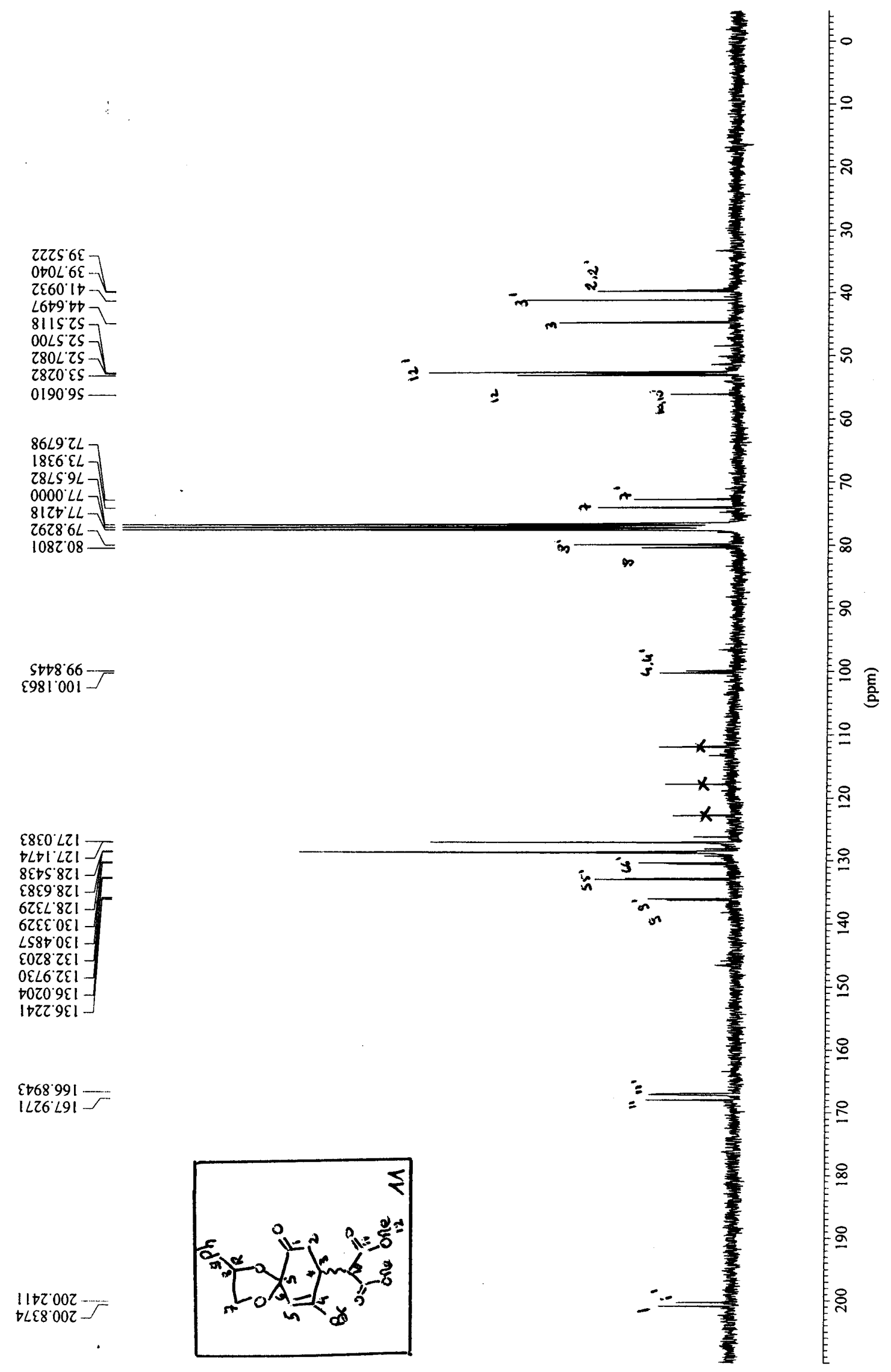

